\title{
Supporting Information Figures to the Paper Titled: "Structure of Proton-bound Methionine and Tryptophan Dimers in the Gas Phase Investigated with IRMPD Spectroscopy and Quantum Chemical Calculations"
}

Åke Andersson, ${ }^{1}$ Mathias Poline, ${ }^{2}$ Meena Kodambattil, ${ }^{3}$ Oleksii Rebrov, ${ }^{2}$ Estelle Loire, ${ }^{4}$ Philipe Maître, ${ }^{4}$ and Vitali Zhaunerchyk ${ }^{5}$

1) Chalmers University of Technology, Gothenburg, Sweden

${ }^{2)}$ Department of Physics, Stockholm University, Stockholm, Sweden

${ }^{3)}$ International School of Photonics, Cochin University of Science and Technology, India

${ }^{4)}$ Laboratoire de Chimie Physique (UMR8000), Université Paris-Sud, CNRS, Université Paris Saclay, Orsay 91405, France

${ }^{5)}$ Department of Physics, University of Gothenburg, Gothenburg, Sweden ${ }^{\text {a) }}$

\section{LIST OF FIGURES}

S1 The stable structures of $\mathrm{Met}_{2} \mathrm{H}^{+} \ldots \ldots \ldots$ S3

S2 $\quad$ The stable structures of $\mathrm{MetTrpH}^{+} \ldots \ldots$. S4

S3 The stable structures of $\operatorname{Trp}_{2} \mathrm{H}^{+} \ldots \ldots$. W5

S4 NCI analyses of $\mathrm{Met}_{2} \mathrm{H}^{+}$conformers ...... S6

S5 $\quad$ NCI analyses of MetTrpH ${ }^{+}$conformers.... S7

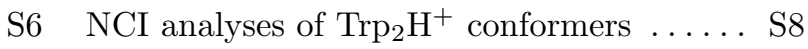

\section{LIST OF TABLES}

S1 Geometry for MM-A1. ................. S9

S2 Geometry for MM-A2. .................S10

S3 Geometry for MM-A3. . ................ S11

S4 Geometry for MM-B1. ..................S12

S5 $\quad$ Geometry for MM-B2. . ................. S13

S6 $\quad$ Geometry for MM-B3. . . . . . . . . . . . . . . . S14

S7 Geometry for MM-B4. ................ S15

S8 $\quad$ Geometry for MM-Z1. ................ S16

S9 $\quad$ Geometry for MM-Z2. . ............... S17

S10 Geometry for MM-Z3. . . . . . . . . . . . . S18

S11 Geometry for MM-Z4. ................. S19

S12 Geometry for MM-Z5. ............... S20

S13 Geometry for MW-A1. ................. S21

a)Electronic mail: vitali.zhaunerchyk@physics.gu.se
S14 Geometry for MW-A2. . ................ S22

S15 Geometry for MW-A3. . ................ S23

S16 Geometry for MW-A4. ................ S24

S17 Geometry for MW-A5. ................. S25

S18 Geometry for MW-A6. . ................ S26

S19 Geometry for MW-A7. . . . . . . . . . . . . . . S27

S20 Geometry for MW-B1. ................. S28

S21 Geometry for MW-Z1. . ..................S29

S22 Geometry for WM-A1. .................S30

S23 Geometry for WM-A2. ................. S31

S24 Geometry for WM-A3. . . . . . . . . . . . . . S32

S25 Geometry for WM-A4. .................. S33

S26 Geometry for WM-A5. . . . . . . . . . . . . . . S34

S27 Geometry for WM-A6. . . . . . . . . . . . . . . S35

S28 Geometry for WM-A7. . ............... S36

S29 Geometry for WM-A8. . . . . . . . . . . . . . S37

S30 Geometry for WM-B1. . ................ S38

S31 Geometry for WM-B2. . ................ S39

S32 Geometry for WM-Z1. . ................ S40

S33 Geometry for WM-Z2. . . . . . . . . . . . . . S41

S34 Geometry for WM-Z3. ................. S42

S35 Geometry for WW-A1. .................. S43

S36 Geometry for WW-A2. .................. S44

S37 Geometry for WW-A3. ................. S45

S38 Geometry for WW-A4. ..................S46

S39 Geometry for WW-A5. ................ S47 
S40 Geometry for WW-A6. . ................. S48

S41 Geometry for WW-B1. ................. S49

S42 Geometry for WW-CS1. . . . . . . . . . . . . . . S50
S43 Geometry for WW-CS4. . ............... S51

S44 Geometry for WW-Z1. ................ S52 


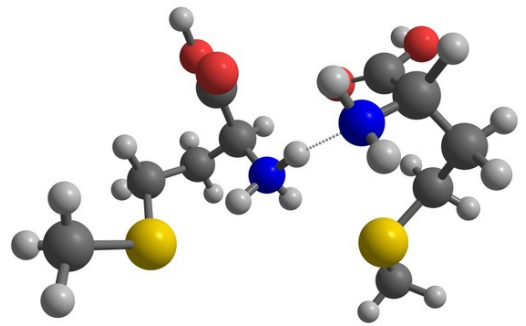

(a) MM-A1 (0)

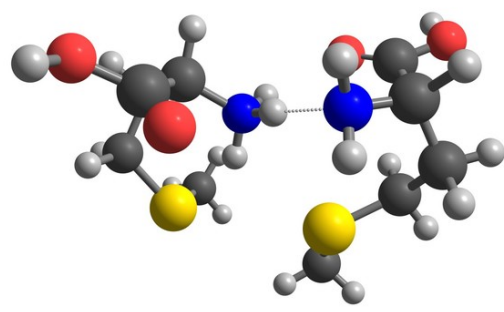

(d) MM-A2 (530)

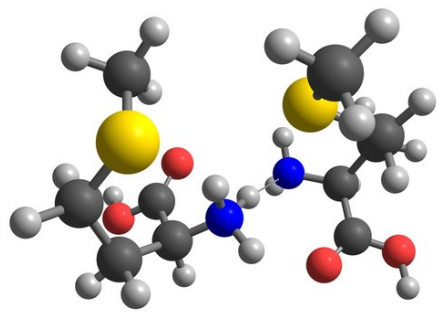

(g) MM-A3 (745)

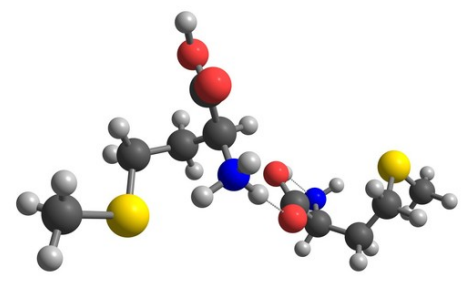

(j) MM-B3 (1130)

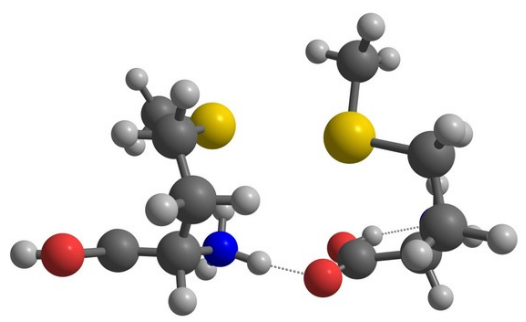

(b) MM-B1 (277)

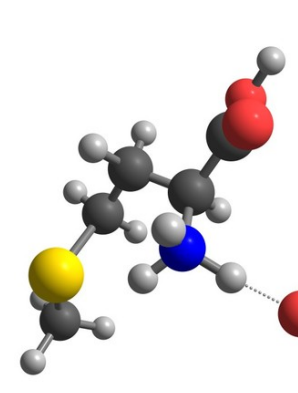

(e) MM-Z1 (622)

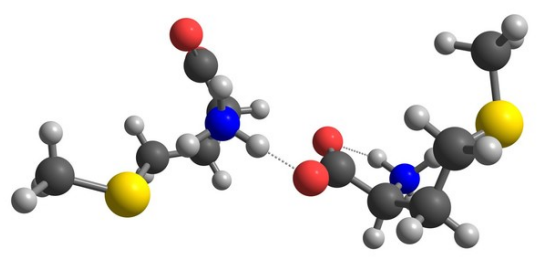

(h) MM-Z3 (836)

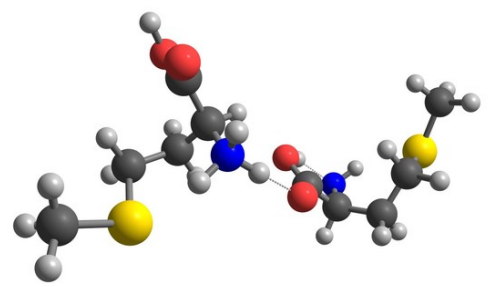

(k) MM-B4 (1415) (c) MM-B2 (321)
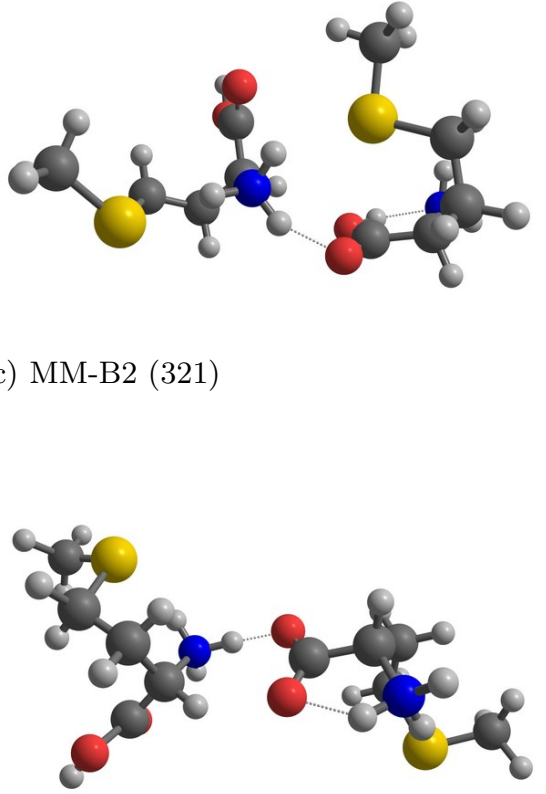

(f) MM-Z2 (626)

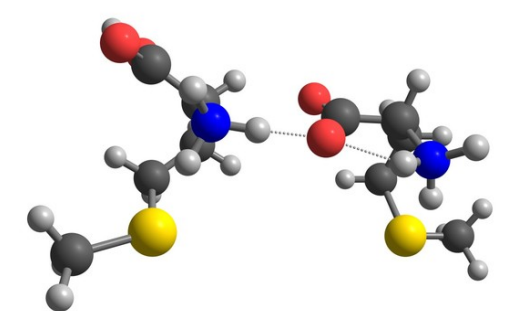

(i) MM-Z4 (860)

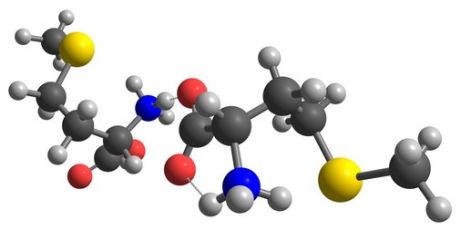

(l) MM-Z5 (1524)

FIG. S1: The stable structures of $\mathrm{Met}_{2} \mathrm{H}^{+}$found in conformational search. Relative electronic energies in $\mathrm{cm}^{-1}$ are shown in parentheses. 


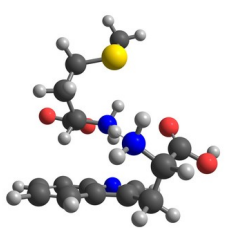

(a) MW-A1 (0)

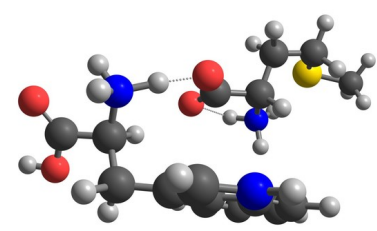

(e) WM-Z1 (1252)

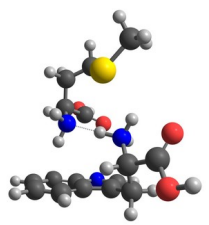

(i) WM-A4 (1374)

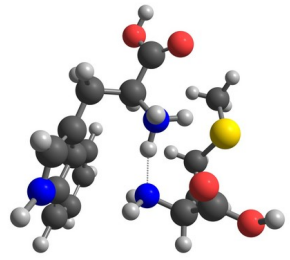

(m) WM-A7 (1614)

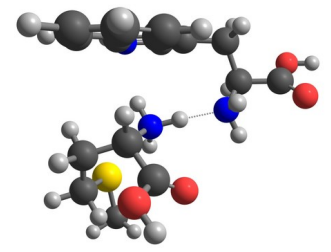

(q) MW-A5 (1820)

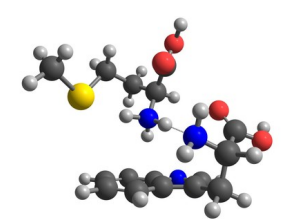

(b) MW-A2 (390)

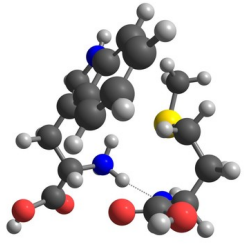

(f) WM-A2 (1317)

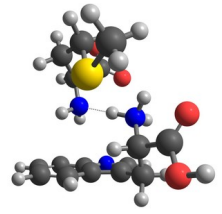

(j) WM-A5 (1455)

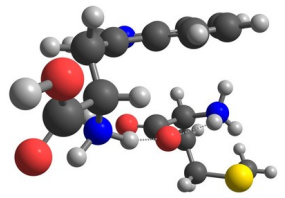

(n) WM-Z3 (1725)

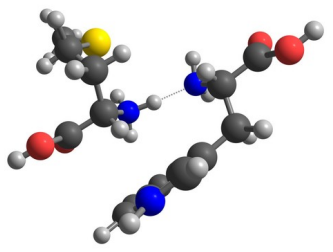

(r) MW-A6 (1953)

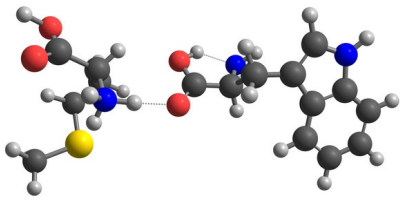
(u) MW-B1 (3648)
(v) MW-Z1 (3804)

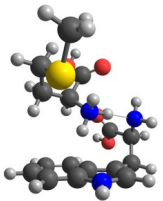

(c) MW-A3 (590)

(d) WM-A1 (882)

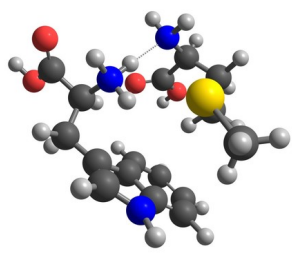

(g) WM-A3 (1318)

(h) WM-Z2 (1332)

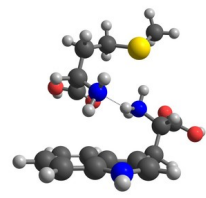

(k) MW-A4 (1560)

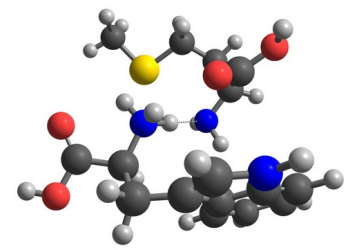

(l) WM-A6 (1597)
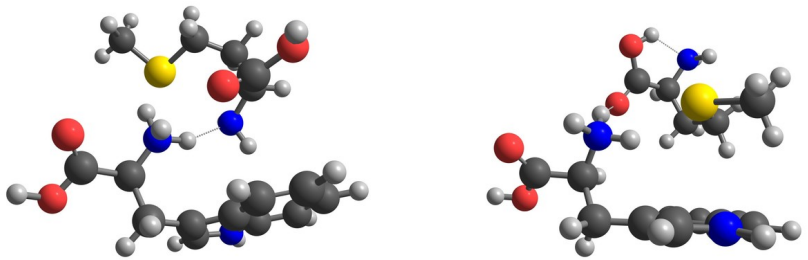

(o) WM-A8 (1796)

(p) WM-B1 (1819)
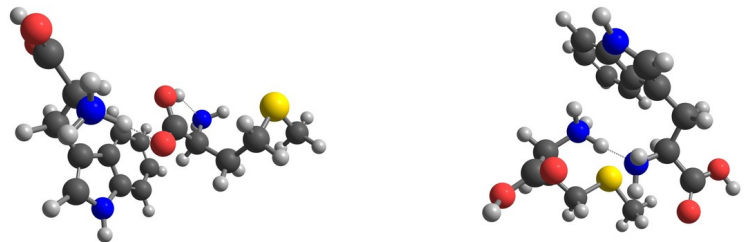

(t) MW-A7 (2738)

(s) WM-B2 (2432)

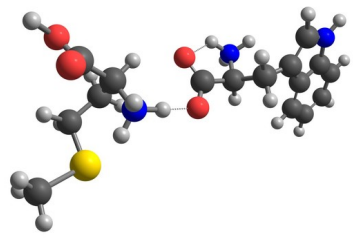

FIG. S2: The stable structures of $\mathrm{MetTrpH}^{+}$found in conformational search. Relative electronic energies in $\mathrm{cm}^{-1}$ are shown in parentheses. 


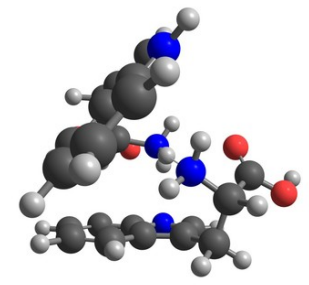

(a) WW-A1 (0)

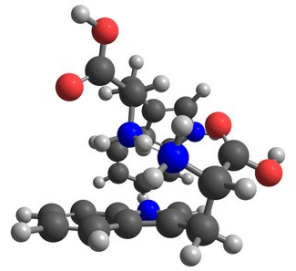

(d) WW-A4 (7)

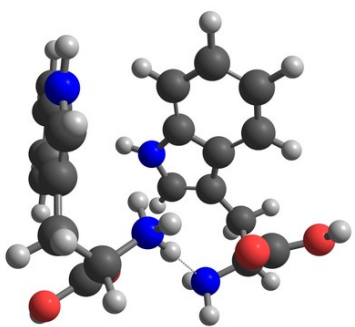

(g) WW-CS1 (21)

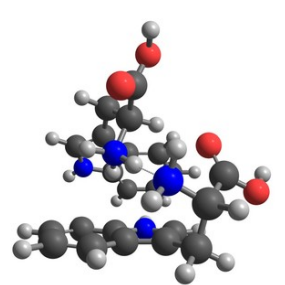

(b) WW-A2 (1)

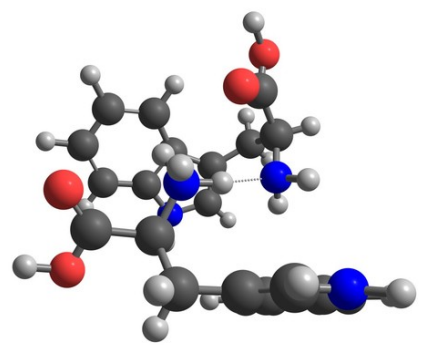

(e) WW-A5 (15)

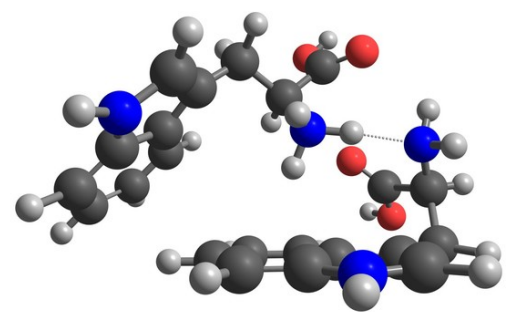

(h) WW-A6 (30)

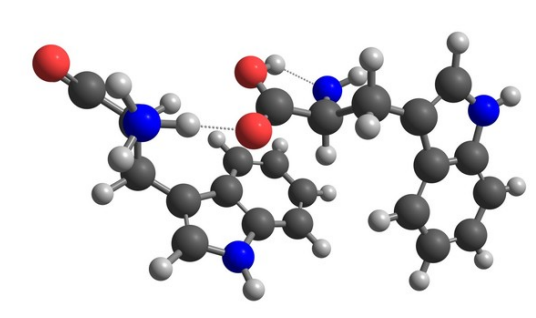

(j) WW-B1 (40)

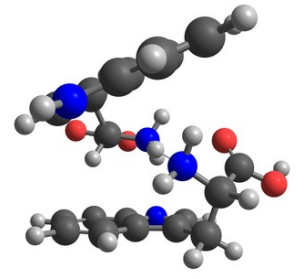

(c) WW-A3 (6)

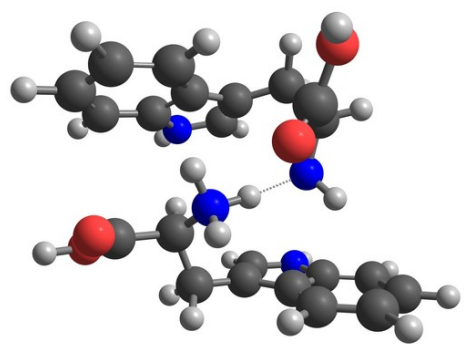

(f) WW-CS4 (21)

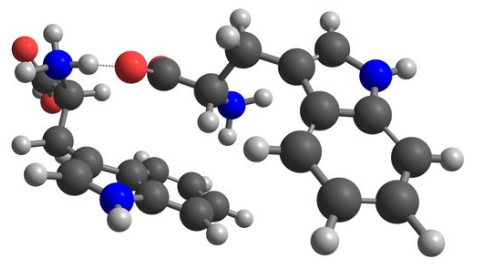

(i) WW-Z1 (31)

FIG. S3: The stable structures of $\operatorname{Trp}_{2} \mathrm{H}^{+}$found in conformational search. Relative electronic energies in $\mathrm{cm}^{-1}$ are shown in parentheses. 

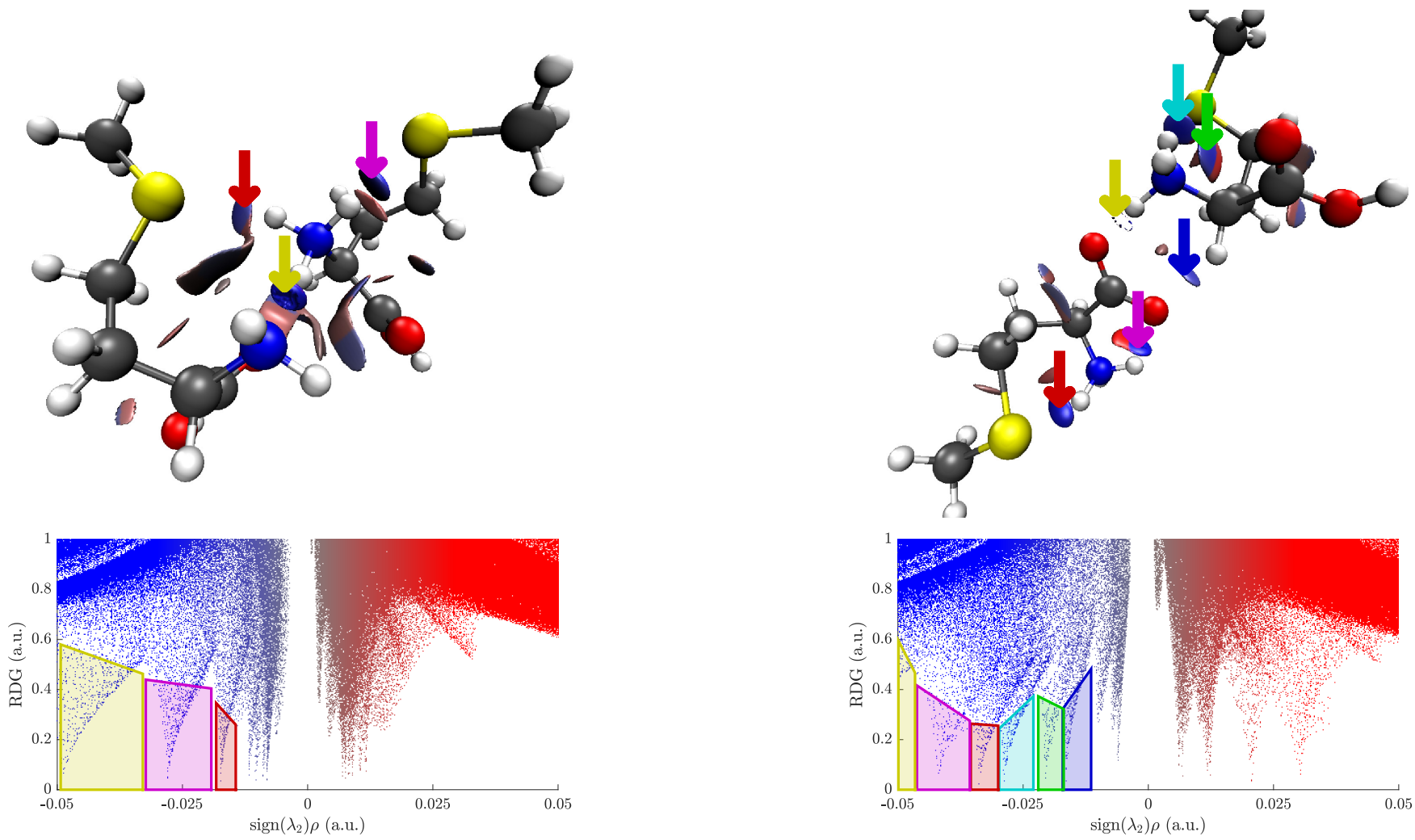

(a) NCI analysis of MM-A1. The strongest interactions are: (yellow) $\mathrm{NH}-\mathrm{n}$. (pink) $\mathrm{NH}-\mathrm{S}$. (red) NH-s.

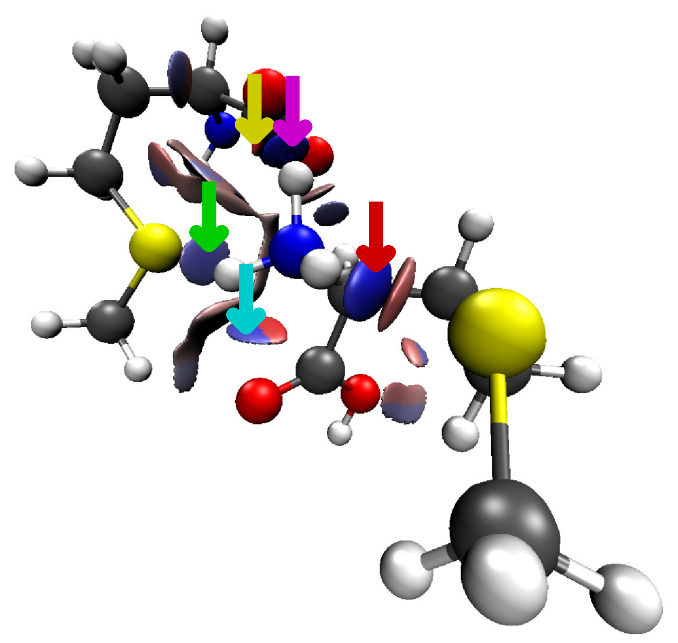

(b) NCI analysis of MM-Z2. The strongest interactions are: (yellow) $\mathrm{NH}-\mathrm{O}$. (pink) nh-o. (red) nh-s. (cyan) NH-S. (green) NH-O. (blue) $\alpha \mathrm{CH}-\mathrm{o}$.
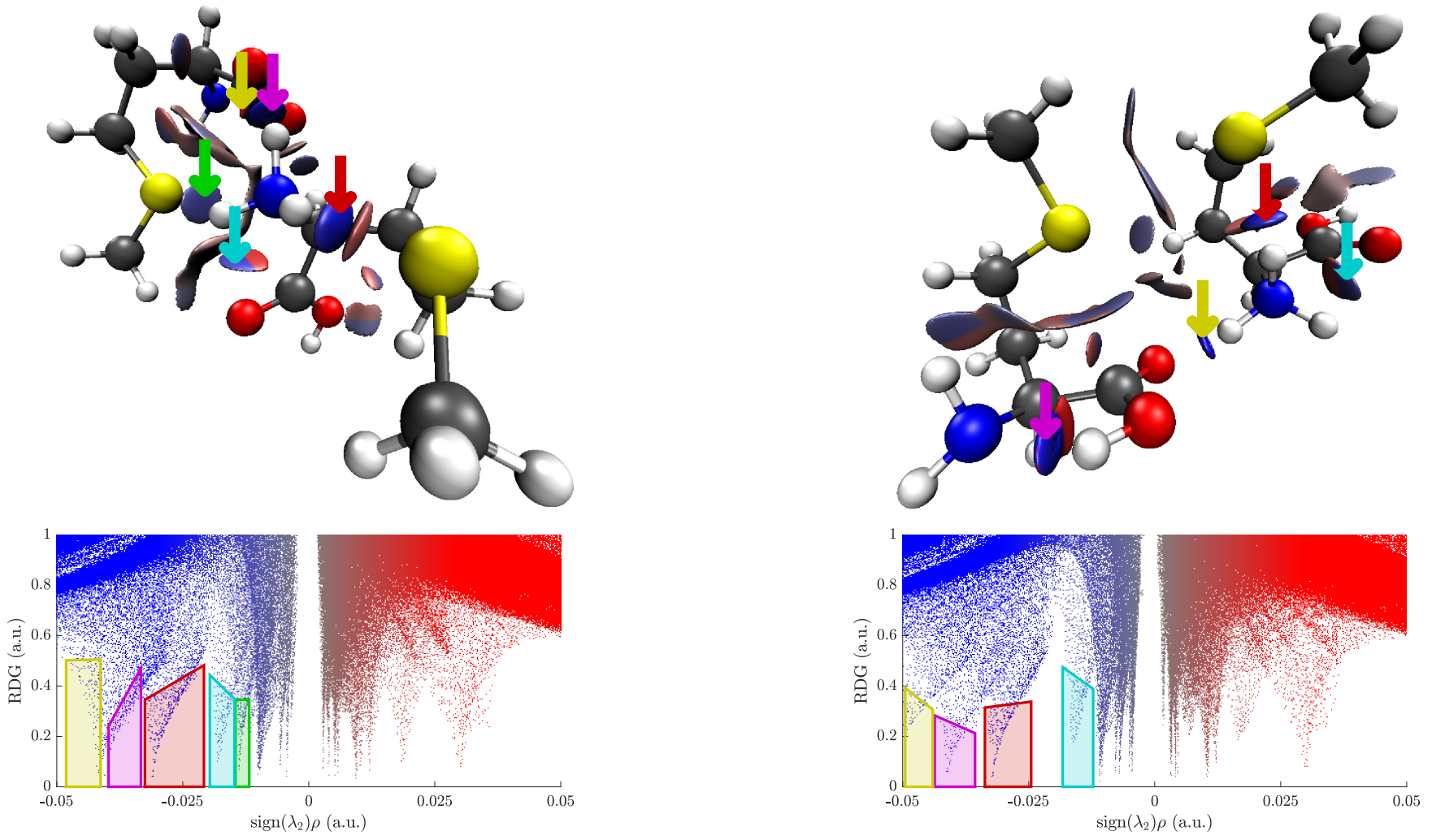

(c) NCI analysis of MM-B2. The strongest interactions are: (yellow) oh-n. (pink) NH-o. (red) NH-S. (cyan) NH-O. (green) NH-s.

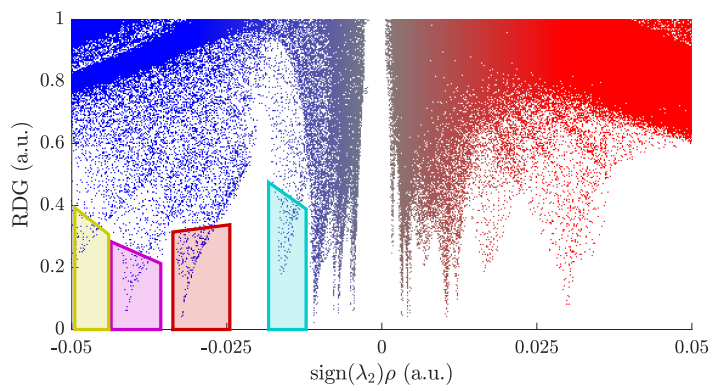

(d) NCI analysis of MM-B1. The strongest interactions are: (yellow) $\mathrm{NH}-\mathrm{O}$. (pink) oh-n. (red) NH-S. (cyan) NH-O.

FIG. S4: NCI analyses of the most abundant $\mathrm{Met}_{2} \mathrm{H}^{+}$conformers. The chemical formulae for interacting groups are written in upper and lower case for the protonated and unprotonated moiety, respectively. 

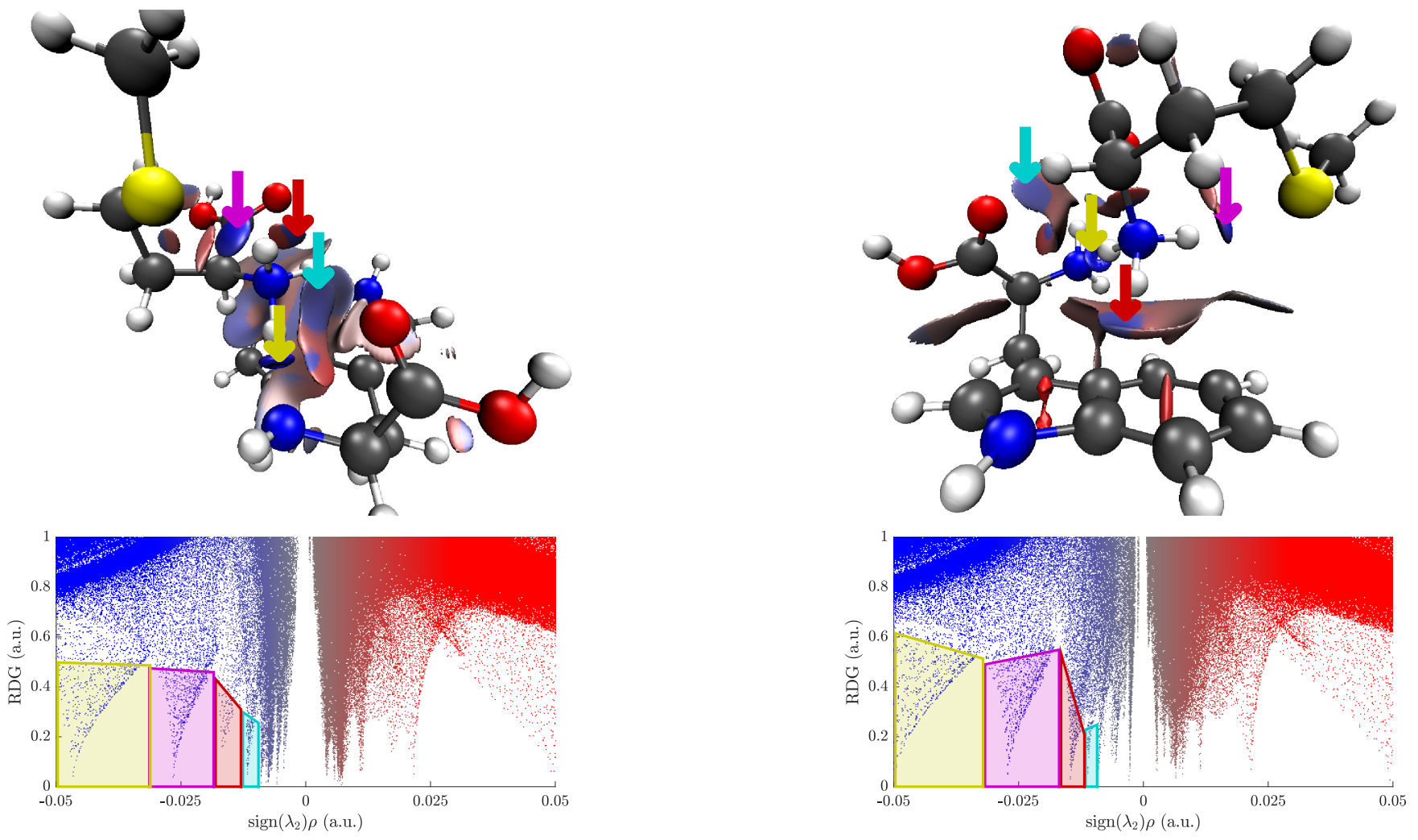

(a) NCI analysis of MW-A1. The strongest interactions are: (yellow) $\mathrm{NH}-\mathrm{n}$. (pink) NH-S. (red) $\mathrm{NH}-\mathrm{O}$. (cyan) $\mathrm{NH}-\mathrm{O}$.
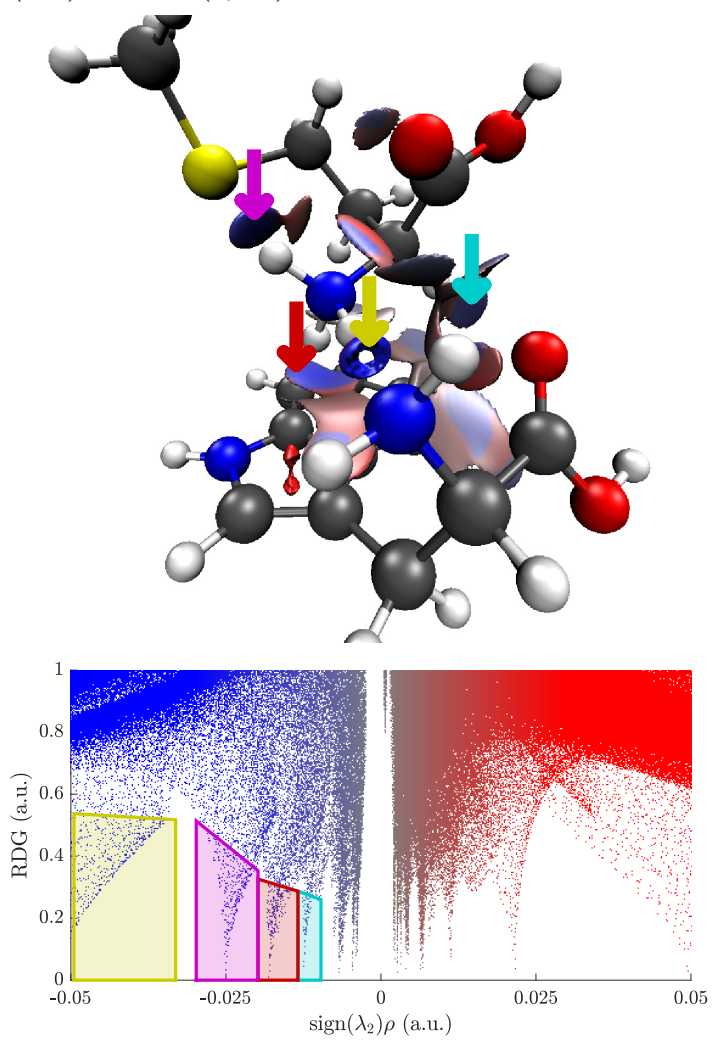

(c) NCI analysis of MW-A3. The strongest interactions are: (yellow) $\mathrm{NH}-n$. (pink) $\mathrm{NH}-\mathrm{S}$. (red) $\mathrm{NH}-\pi$. (cyan) $\alpha \mathrm{CH}-\mathrm{o}$.

(b) NCI analysis of MW-A2. The strongest interactions are: (yellow) $\mathrm{NH}-\mathrm{n}$. (pink) NH-S. (red) $\mathrm{NH}-\pi$. (cyan) $\alpha \mathrm{CH}-\mathrm{o}$.
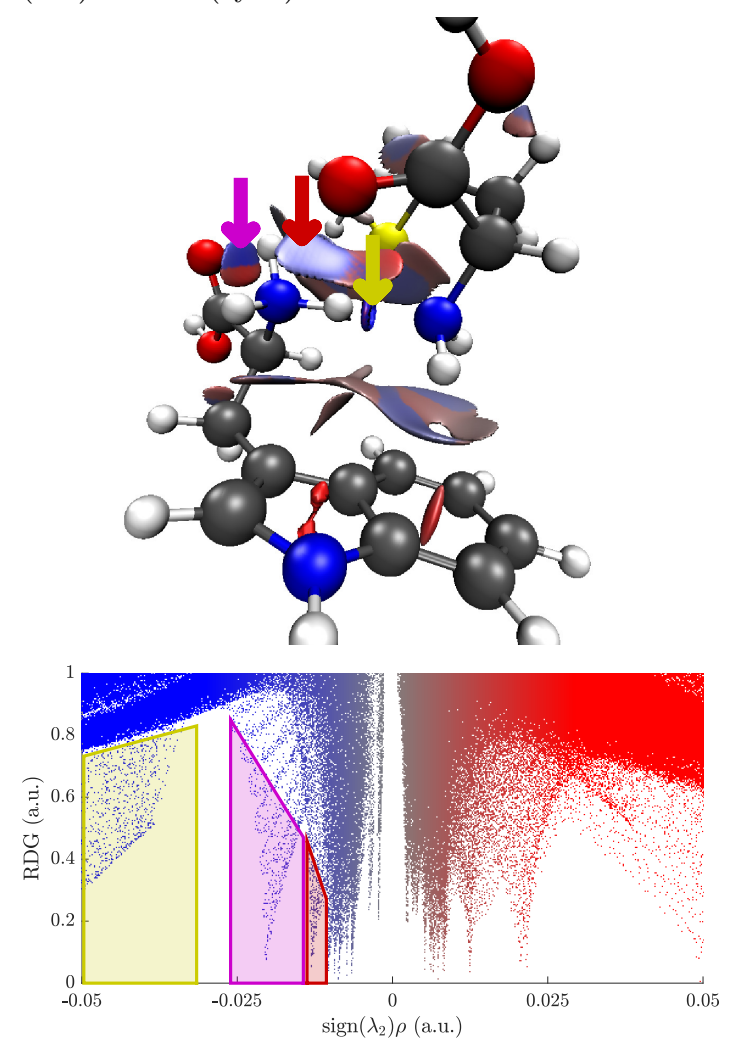

(d) NCI analysis of WM-A5. The strongest interactions are: (yellow) $\mathrm{NH}-n$. (pink) $\mathrm{NH}-\mathrm{O}$. (red) $\mathrm{NH}-\mathrm{O}$.

FIG. S5: NCI analyses of the most abundant MetTrpH ${ }^{+}$conformers. The chemical formulae for interacting groups are written in upper and lower case for the protonated and unprotonated moiety, respectively. 

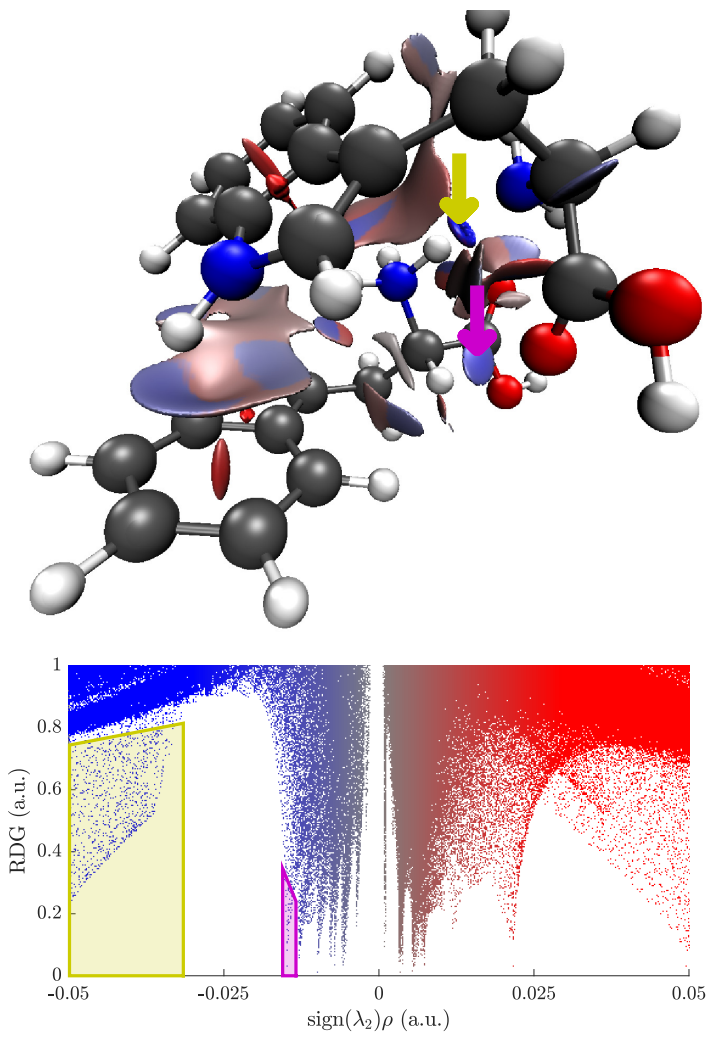

(a) NCI analysis of WW-A2. The strongest interactions are: (yellow) $\mathrm{NH}-\mathrm{n}$. (pink) $\alpha \mathrm{CH}-\mathrm{o}$.
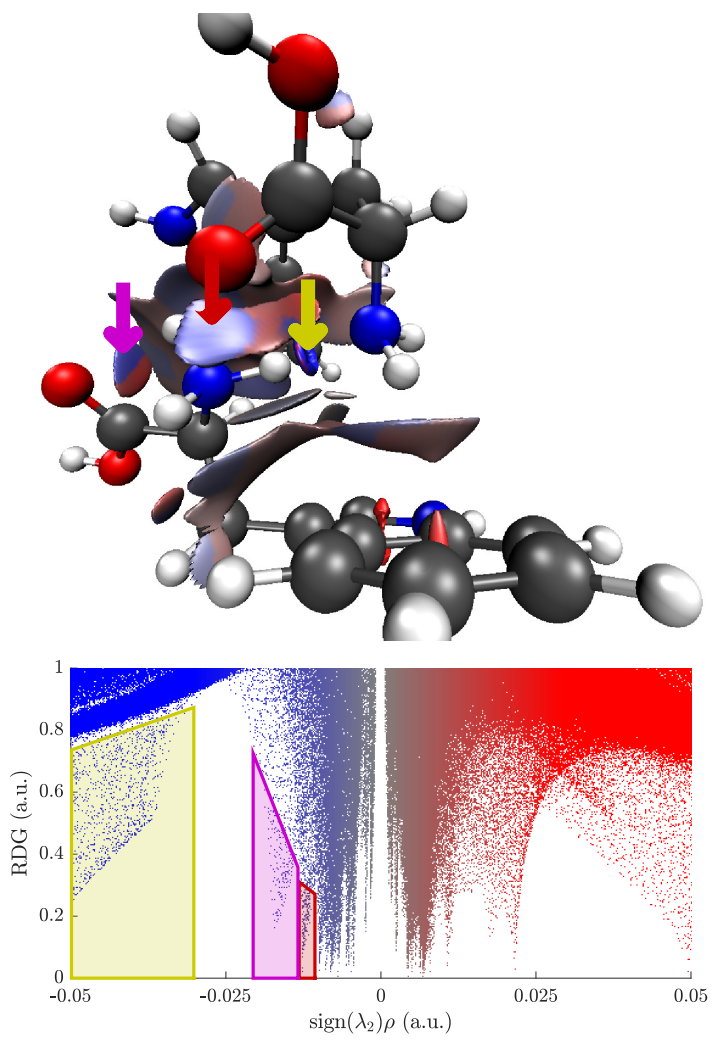

(c) NCI analysis of WW-A3. The strongest interactions are: (yellow) $\mathrm{NH}-\mathrm{n}$. (pink) $\mathrm{NH}-\mathrm{O}$. (red) $\mathrm{NH}-\mathrm{O}$.
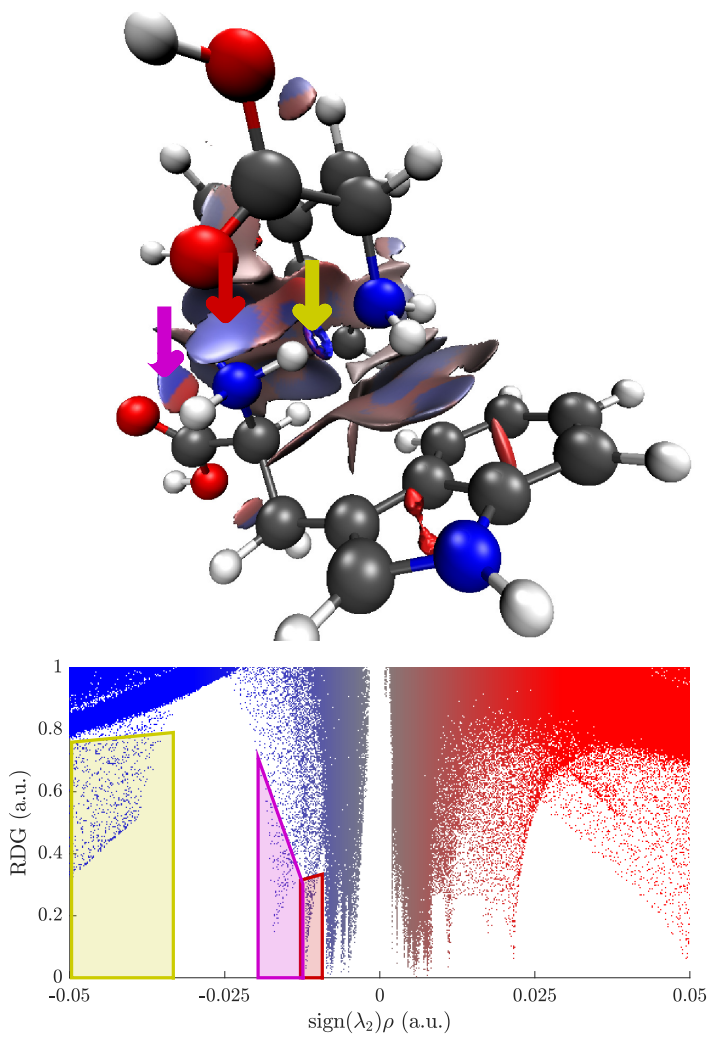

(b) NCI analysis of WW-A1. The strongest interactions are: (yellow) NH-n. (pink) $\mathrm{NH}-\mathrm{O}$. (red) $\mathrm{NH}-\mathrm{O}$.
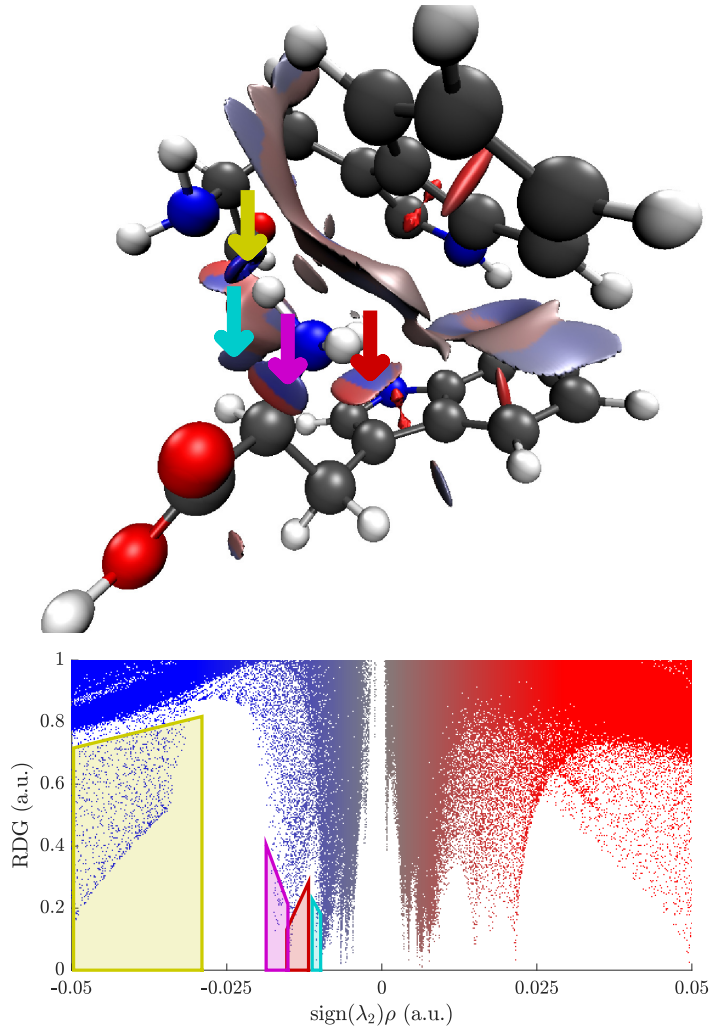

(d) NCI analysis of WW-A4. The strongest interactions are: (yellow) NH-n. (pink) $\mathrm{NH}-\mathrm{O}$. (red) $\mathrm{NH}-\pi$. (cyan) $\mathrm{NH}-\mathrm{O}$.

FIG. S6: NCI analyses of the most abundant $\operatorname{Trp}_{2} \mathrm{H}^{+}$conformers. The chemical formulae for interacting groups are written in upper and lower case for the protonated and unprotonated moiety, respectively. 
TABLE S1: Geometry for MM-A1.

\begin{tabular}{lrrr} 
Element & X & $\mathrm{Y}$ & $\mathrm{Z}$ \\
\hline $\mathrm{S}$ & -3.451652000 & -1.602336000 & 0.278154000 \\
$\mathrm{~S}$ & 1.985464000 & -2.241748000 & -0.026837000 \\
$\mathrm{O}$ & -2.086647000 & 2.914821000 & -0.816052000 \\
$\mathrm{O}$ & 3.567069000 & 2.464056000 & -0.386609000 \\
$\mathrm{O}$ & 1.453642000 & 1.792038000 & -0.739865000 \\
$\mathrm{O}$ & -1.389769000 & 1.914223000 & 1.078325000 \\
$\mathrm{C}$ & -3.745730000 & -0.118061000 & -0.776874000 \\
$\mathrm{C}$ & -1.291471000 & 0.707166000 & -1.014524000 \\
$\mathrm{C}$ & 2.461744000 & 1.778787000 & -0.071242000 \\
$\mathrm{C}$ & 3.657383000 & -0.175211000 & 0.958056000 \\
$\mathrm{C}$ & 2.656684000 & 0.974306000 & 1.205512000 \\
$\mathrm{C}$ & 3.404831000 & -1.088448000 & -0.243608000 \\
$\mathrm{C}$ & -2.560687000 & 0.172221000 & -1.702490000 \\
$\mathrm{C}$ & 2.478600000 & -3.561712000 & -1.188460000 \\
$\mathrm{C}$ & -4.541614000 & -1.223757000 & 1.692235000 \\
$\mathrm{C}$ & -1.579677000 & 1.898423000 & -0.112092000 \\
$\mathrm{H}$ & -5.577657000 & -1.162010000 & 1.360547000 \\
$\mathrm{H}$ & 4.637993000 & 0.284753000 & 0.816013000 \\
$\mathrm{H}$ & -0.085814000 & -1.007989000 & -0.743316000 \\
$\mathrm{H}$ & 3.126832000 & 1.647198000 & 1.931762000 \\
$\mathrm{H}$ & 3.267974000 & -0.512373000 & -1.161898000 \\
$\mathrm{H}$ & 4.285242000 & -1.718407000 & -0.379367000 \\
$\mathrm{H}$ & 0.116974000 & 0.072550000 & 0.482348000 \\
$\mathrm{H}$ & -3.942292000 & 0.731310000 & -0.118515000 \\
$\mathrm{H}$ & 3.722225000 & -0.772975000 & 1.871901000 \\
$\mathrm{H}$ & -4.641061000 & -0.290155000 & -1.374705000 \\
$\mathrm{H}$ & -0.577087000 & 1.039818000 & -1.765919000 \\
$\mathrm{H}$ & -2.298002000 & -0.719654000 & -2.280086000 \\
$\mathrm{H}$ & 1.694199000 & -4.317654000 & -1.159860000 \\
$\mathrm{H}$ & 2.569881000 & -3.175957000 & -2.203747000 \\
$\mathrm{H}$ & 0.811217000 & 1.253837000 & 2.082503000 \\
$\mathrm{H}$ & 1.482376000 & -0.202189000 & 2.404142000 \\
$\mathrm{H}$ & -2.869149000 & 0.927989000 & -2.427656000 \\
$\mathrm{H}$ & 3.418099000 & -4.011956000 & -0.868157000 \\
$\mathrm{H}$ & -1.322112000 & -0.848014000 & 0.364391000 \\
$\mathrm{H}$ & -4.442743000 & -2.051531000 & 2.393590000 \\
$\mathrm{H}$ & -4.237692000 & -0.296523000 & 2.177806000 \\
$\mathrm{~N}$ & -0.611902000 & -0.326675000 & -0.183206000 \\
$\mathrm{~N}$ & 1.350303000 & 0.492878000 & 1.674769000 \\
$\mathrm{H}$ & 3.411878000 & 2.949179000 & -1.212472000 \\
$\mathrm{H}$ & -2.255826000 & 3.659314000 & -0.216765000 \\
& & &
\end{tabular}


TABLE S2: Geometry for MM-A2.

\begin{tabular}{lrrr} 
Element & $\mathrm{X}$ & $\mathrm{Y}$ & $\mathrm{Z}$ \\
\hline $\mathrm{S}$ & -2.660392000 & 1.721479000 & -0.094873000 \\
$\mathrm{~S}$ & 1.221909000 & 1.724003000 & -1.029649000 \\
$\mathrm{O}$ & -3.140832000 & -2.801108000 & -0.840673000 \\
$\mathrm{O}$ & -1.353940000 & -1.836191000 & -1.821089000 \\
$\mathrm{O}$ & 1.850560000 & -1.163785000 & 1.758680000 \\
$\mathrm{O}$ & 4.086176000 & -1.311053000 & 1.689106000 \\
$\mathrm{C}$ & -3.651613000 & 0.248790000 & 0.383001000 \\
$\mathrm{C}$ & -2.036191000 & -2.045967000 & -0.852321000 \\
$\mathrm{C}$ & -2.485276000 & 2.545946000 & 1.525490000 \\
$\mathrm{C}$ & 0.972756000 & 3.483064000 & -0.620175000 \\
$\mathrm{C}$ & -2.933072000 & -0.817902000 & 1.215754000 \\
$\mathrm{C}$ & 2.888866000 & 1.461663000 & -0.302435000 \\
$\mathrm{C}$ & 2.997806000 & -1.165366000 & -0.399328000 \\
$\mathrm{C}$ & 3.580437000 & 0.201435000 & -0.826242000 \\
$\mathrm{C}$ & 2.879317000 & -1.219777000 & 1.120635000 \\
$\mathrm{C}$ & -1.732506000 & -1.503037000 & 0.540770000 \\
$\mathrm{H}$ & -1.953781000 & 3.479541000 & 1.343496000 \\
$\mathrm{H}$ & -3.289619000 & -3.155183000 & -1.732456000 \\
$\mathrm{H}$ & 3.981156000 & -1.316495000 & 2.653875000 \\
$\mathrm{H}$ & -1.912030000 & 1.947145000 & 2.234356000 \\
$\mathrm{H}$ & -3.467788000 & 2.774592000 & 1.938769000 \\
$\mathrm{H}$ & -0.846283000 & 0.329818000 & 0.063459000 \\
$\mathrm{H}$ & 1.736493000 & 4.098256000 & -1.096223000 \\
$\mathrm{H}$ & -3.655791000 & -1.599448000 & 1.460787000 \\
$\mathrm{H}$ & 1.641351000 & -1.046625000 & -1.948783000 \\
$\mathrm{H}$ & 1.575101000 & -2.455634000 & -1.144506000 \\
$\mathrm{H}$ & 0.982740000 & 3.640231000 & 0.459054000 \\
$\mathrm{H}$ & -0.003268000 & 3.760419000 & -1.018066000 \\
$\mathrm{H}$ & -2.597065000 & -0.401273000 & 2.169665000 \\
$\mathrm{H}$ & -1.426900000 & -2.357013000 & 1.151560000 \\
$\mathrm{H}$ & -4.534139000 & 0.597569000 & 0.921990000 \\
$\mathrm{H}$ & 3.615285000 & 0.223556000 & -1.919417000 \\
$\mathrm{H}$ & -4.004398000 & -0.169483000 & -0.561746000 \\
$\mathrm{H}$ & 0.221969000 & -0.964066000 & -0.204095000 \\
$\mathrm{H}$ & 3.498525000 & 2.323006000 & -0.581082000 \\
$\mathrm{H}$ & 2.812851000 & 1.448494000 & 0.788189000 \\
$\mathrm{H}$ & 3.743999000 & -1.916518000 & -0.677874000 \\
$\mathrm{H}$ & -0.089326000 & -0.471154000 & 1.325068000 \\
$\mathrm{H}$ & 4.616142000 & 0.226143000 & -0.480291000 \\
$\mathrm{~N}$ & -0.557966000 & -0.597266000 & 0.425985000 \\
$\mathrm{~N}$ & 1.693920000 & -1.454103000 & -1.019195000
\end{tabular}


TABLE S3: Geometry for MM-A3.

\begin{tabular}{lrrr} 
Element & $\mathrm{X}$ & $\mathrm{Y}$ & $\mathrm{Z}$ \\
\hline $\mathrm{H}$ & -2.122108000 & 2.834523000 & -1.448015000 \\
$\mathrm{C}$ & -2.693601000 & 1.940746000 & -1.198236000 \\
$\mathrm{H}$ & -2.270264000 & 1.086935000 & -1.725600000 \\
$\mathrm{~S}$ & -2.562378000 & 1.739893000 & 0.616382000 \\
$\mathrm{H}$ & -3.732863000 & 2.097885000 & -1.484308000 \\
$\mathrm{H}$ & -0.786059000 & 0.338414000 & 0.556555000 \\
$\mathrm{H}$ & 0.192279000 & -0.861730000 & -0.151523000 \\
$\mathrm{~N}$ & -0.494931000 & -0.654299000 & 0.639091000 \\
$\mathrm{H}$ & 0.072633000 & -0.760297000 & 1.481837000 \\
$\mathrm{O}$ & -1.566453000 & -1.364914000 & -1.739635000 \\
$\mathrm{C}$ & -3.588008000 & 0.224834000 & 0.896089000 \\
$\mathrm{C}$ & -1.688043000 & -1.544474000 & 0.668688000 \\
$\mathrm{H}$ & -4.031924000 & -0.053008000 & -0.061156000 \\
$\mathrm{C}$ & -2.108995000 & -1.826170000 & -0.767743000 \\
$\mathrm{C}$ & -2.829536000 & -0.952798000 & 1.517114000 \\
$\mathrm{H}$ & -2.417821000 & -0.667891000 & 2.489877000 \\
$\mathrm{H}$ & -4.405466000 & 0.505458000 & 1.560003000 \\
$\mathrm{H}$ & -1.378856000 & -2.496632000 & 1.106513000 \\
$\mathrm{O}$ & -3.158524000 & -2.653771000 & -0.799722000 \\
$\mathrm{H}$ & -3.545120000 & -1.756147000 & 1.706460000 \\
$\mathrm{H}$ & -3.398212000 & -2.826420000 & -1.724625000 \\
$\mathrm{H}$ & 0.023953000 & 3.855030000 & 0.397755000 \\
$\mathrm{H}$ & 1.743227000 & 4.234632000 & 0.163104000 \\
$\mathrm{C}$ & 1.040365000 & 3.483096000 & 0.522843000 \\
$\mathrm{~S}$ & 1.176953000 & 1.955909000 & -0.465497000 \\
$\mathrm{H}$ & 3.345528000 & 0.670849000 & -2.017663000 \\
$\mathrm{H}$ & 1.213563000 & 3.278159000 & 1.579612000 \\
$\mathrm{H}$ & 1.351990000 & -0.565299000 & -2.067530000 \\
$\mathrm{H}$ & 3.519322000 & 2.385707000 & -0.200779000 \\
$\mathrm{C}$ & 2.908432000 & 1.491612000 & -0.064116000 \\
$\mathrm{C}$ & 3.465717000 & 0.390014000 & -0.967033000 \\
$\mathrm{~N}$ & 1.511600000 & -1.167220000 & -1.264634000 \\
$\mathrm{H}$ & 4.540490000 & 0.328178000 & -0.783334000 \\
$\mathrm{H}$ & 1.335713000 & -2.115273000 & -1.585918000 \\
$\mathrm{C}$ & 2.898257000 & -1.036310000 & -0.788278000 \\
$\mathrm{H}$ & 2.968152000 & 1.207940000 & 0.990156000 \\
$\mathrm{H}$ & 3.570827000 & -1.704575000 & -1.336205000 \\
$\mathrm{C}$ & 2.984537000 & -1.447991000 & 0.678107000 \\
$\mathrm{O}$ & 2.050073000 & -1.566570000 & 1.439766000 \\
$\mathrm{O}$ & 4.255659000 & -1.646028000 & 1.043435000 \\
$\mathrm{H}$ & 4.280356000 & -1.883725000 & 1.984060000
\end{tabular}


TABLE S4: Geometry for MM-B1.

\begin{tabular}{lrrr} 
Element & $\mathrm{X}$ & $\mathrm{Y}$ & $\mathrm{Z}$ \\
\hline $\mathrm{H}$ & 5.275719000 & -1.240120000 & -1.161602000 \\
$\mathrm{H}$ & 2.030216000 & -0.164193000 & -2.471189000 \\
$\mathrm{H}$ & 1.889455000 & -2.182318000 & -1.255088000 \\
$\mathrm{H}$ & 3.245705000 & 1.338510000 & -0.907501000 \\
$\mathrm{H}$ & 1.802461000 & 2.090019000 & -1.577343000 \\
$\mathrm{H}$ & 0.567736000 & -0.051916000 & -1.507100000 \\
$\mathrm{H}$ & 1.315330000 & -0.475756000 & 1.072658000 \\
$\mathrm{H}$ & 1.797776000 & -2.067262000 & 1.259975000 \\
$\mathrm{H}$ & 3.632142000 & 2.953829000 & 1.128185000 \\
$\mathrm{H}$ & 2.921944000 & 2.367500000 & 2.643573000 \\
$\mathrm{H}$ & 3.833265000 & 1.238371000 & 1.616207000 \\
$\mathrm{H}$ & 0.348651000 & -1.715277000 & 0.411387000 \\
$\mathrm{H}$ & -3.295076000 & 1.335219000 & -2.234140000 \\
$\mathrm{H}$ & -2.058691000 & 3.613665000 & -1.127562000 \\
$\mathrm{H}$ & -2.835861000 & 3.334573000 & 0.458571000 \\
$\mathrm{H}$ & -2.831438000 & -1.290259000 & 2.169391000 \\
$\mathrm{H}$ & -4.297900000 & 0.213046000 & 1.104403000 \\
$\mathrm{H}$ & -4.127094000 & 1.389975000 & -0.685891000 \\
$\mathrm{H}$ & -1.068167000 & 3.486307000 & 0.343910000 \\
$\mathrm{H}$ & -5.238781000 & -1.137985000 & 1.149835000 \\
$\mathrm{H}$ & -2.777913000 & -1.052004000 & -1.977969000 \\
$\mathrm{H}$ & -4.500921000 & -0.764843000 & -1.802489000 \\
$\mathrm{H}$ & -3.907202000 & -2.370019000 & -0.185810000 \\
$\mathrm{~N}$ & 1.339449000 & -1.416180000 & 0.619595000 \\
$\mathrm{~N}$ & -4.284564000 & -0.800915000 & 1.121792000 \\
$\mathrm{C}$ & -1.963057000 & 3.121872000 & -0.159380000 \\
$\mathrm{C}$ & -3.309719000 & 0.908353000 & -1.229973000 \\
$\mathrm{C}$ & 1.658600000 & -0.047536000 & -1.450620000 \\
$\mathrm{C}$ & 3.165130000 & 2.099050000 & 1.615790000 \\
$\mathrm{C}$ & -2.091456000 & -1.631140000 & 0.518480000 \\
$\mathrm{C}$ & 2.103759000 & -1.290687000 & -0.661515000 \\
$\mathrm{C}$ & -3.513698000 & -1.366543000 & 0.010879000 \\
$\mathrm{C}$ & 2.155509000 & 1.298219000 & -0.917414000 \\
$\mathrm{C}$ & -3.529952000 & -0.601379000 & -1.327315000 \\
$\mathrm{C}$ & 3.586171000 & -1.301963000 & -0.316086000 \\
$\mathrm{~S}$ & 1.578428000 & 1.696841000 & 0.798058000 \\
$\mathrm{~S}$ & -1.740339000 & 1.324529000 & -0.371296000 \\
$\mathrm{O}$ & 4.006750000 & -1.396331000 & 0.809024000 \\
$\mathrm{O}$ & 4.338813000 & -1.198474000 & -1.413964000 \\
$\mathrm{O}$ & -1.161390000 & -1.926483000 & -0.219040000 \\
$\mathrm{O}$ & -1.944508000 & -1.578810000 & 1.826556000
\end{tabular}


TABLE S5: Geometry for MM-B2.

\begin{tabular}{lrrr} 
Element & $\mathrm{X}$ & $\mathrm{Y}$ & $\mathrm{Z}$ \\
\hline $\mathrm{H}$ & 1.892653000 & 2.466999000 & 2.539282000 \\
$\mathrm{H}$ & 2.452426000 & -1.024075000 & 2.346332000 \\
$\mathrm{H}$ & 0.346051000 & -0.482836000 & 1.361329000 \\
$\mathrm{H}$ & 3.967639000 & 0.418130000 & 0.858687000 \\
$\mathrm{H}$ & 4.571387000 & -1.191893000 & 1.248376000 \\
$\mathrm{H}$ & 2.188680000 & -2.093616000 & 0.987848000 \\
$\mathrm{H}$ & 1.826529000 & -0.412570000 & -1.172768000 \\
$\mathrm{H}$ & 0.430814000 & 0.526681000 & -0.970906000 \\
$\mathrm{H}$ & 6.111022000 & 0.084352000 & -1.061142000 \\
$\mathrm{H}$ & 5.333264000 & 0.117629000 & -2.655035000 \\
$\mathrm{H}$ & 4.790212000 & 1.249176000 & -1.393298000 \\
$\mathrm{H}$ & 0.304800000 & -1.117348000 & -0.780852000 \\
$\mathrm{H}$ & -3.717226000 & 1.368069000 & -2.146064000 \\
$\mathrm{H}$ & -2.326887000 & 3.558298000 & -1.409373000 \\
$\mathrm{H}$ & -2.915909000 & 3.433630000 & 0.276092000 \\
$\mathrm{H}$ & -2.576371000 & -1.486784000 & 1.935803000 \\
$\mathrm{H}$ & -4.291555000 & -0.047021000 & 1.190003000 \\
$\mathrm{H}$ & -4.355551000 & 1.292390000 & -0.508484000 \\
$\mathrm{H}$ & -1.169818000 & 3.537667000 & -0.061385000 \\
$\mathrm{H}$ & -5.098779000 & -1.477563000 & 1.289697000 \\
$\mathrm{H}$ & -3.102431000 & -0.995577000 & -2.135762000 \\
$\mathrm{H}$ & -4.794443000 & -0.794155000 & -1.710691000 \\
$\mathrm{H}$ & -3.926714000 & -2.499459000 & -0.332499000 \\
$\mathrm{~N}$ & 0.938794000 & -0.304240000 & -0.638774000 \\
$\mathrm{~N}$ & -4.190600000 & -1.055014000 & 1.142488000 \\
$\mathrm{C}$ & -2.124261000 & 3.152544000 & -0.418377000 \\
$\mathrm{C}$ & -3.595523000 & 0.879982000 & -1.177836000 \\
$\mathrm{C}$ & 2.418931000 & -1.058175000 & 1.255870000 \\
$\mathrm{C}$ & 5.169781000 & 0.245728000 & -1.585585000 \\
$\mathrm{C}$ & -2.058862000 & -1.714536000 & 0.180678000 \\
$\mathrm{C}$ & 1.243273000 & -0.168663000 & 0.822684000 \\
$\mathrm{C}$ & -3.552157000 & -1.496810000 & -0.101630000 \\
$\mathrm{C}$ & 3.791488000 & -0.650157000 & 0.713153000 \\
$\mathrm{C}$ & -3.773273000 & -0.628664000 & -1.357100000 \\
$\mathrm{C}$ & 1.448606000 & 1.312036000 & 1.111390000 \\
$\mathrm{~S}$ & 3.952323000 & -1.020473000 & -1.088202000 \\
$\mathrm{~S}$ & -1.950044000 & 1.340267000 & -0.500228000 \\
$\mathrm{O}$ & 1.288212000 & 2.183004000 & 0.293211000 \\
$\mathrm{O}$ & 1.799839000 & 1.513199000 & 2.383757000 \\
$\mathrm{O}$ & -1.239240000 & -1.913450000 & -0.697387000 \\
$\mathrm{O}$ & -1.726515000 & -1.691723000 & 1.462197000
\end{tabular}


TABLE S6: Geometry for MM-B3.

\begin{tabular}{lrrr} 
Element & $\mathrm{X}$ & $\mathrm{Y}$ & $\mathrm{Z}$ \\
\hline $\mathrm{H}$ & 3.631855000 & 3.687418000 & 0.654176000 \\
$\mathrm{H}$ & 2.566310000 & 0.714227000 & 2.236812000 \\
$\mathrm{H}$ & 0.980933000 & 1.335861000 & 0.565618000 \\
$\mathrm{H}$ & 4.631172000 & 0.418974000 & 0.718390000 \\
$\mathrm{H}$ & 4.416369000 & -0.777412000 & 1.995614000 \\
$\mathrm{H}$ & 1.887044000 & -0.730156000 & 1.523866000 \\
$\mathrm{H}$ & 2.467787000 & -0.508953000 & -1.153464000 \\
$\mathrm{H}$ & 1.786374000 & 0.853127000 & -1.843922000 \\
$\mathrm{H}$ & 6.431896000 & -1.740957000 & -0.065237000 \\
$\mathrm{H}$ & 5.817535000 & -2.313297000 & -1.628207000 \\
$\mathrm{H}$ & 5.847994000 & -0.566955000 & -1.286675000 \\
$\mathrm{H}$ & 0.777019000 & -0.260874000 & -1.027063000 \\
$\mathrm{H}$ & -2.972776000 & 0.642556000 & -1.426246000 \\
$\mathrm{H}$ & -6.851416000 & 0.608127000 & -1.211712000 \\
$\mathrm{H}$ & -6.547570000 & -0.554332000 & 0.112693000 \\
$\mathrm{H}$ & -1.659931000 & -0.380150000 & 1.883285000 \\
$\mathrm{H}$ & -3.923613000 & -0.398061000 & 1.586671000 \\
$\mathrm{H}$ & -4.476609000 & 0.157176000 & -2.181023000 \\
$\mathrm{H}$ & -7.133193000 & 1.079752000 & 0.475362000 \\
$\mathrm{H}$ & -3.597832000 & -1.900119000 & 2.218832000 \\
$\mathrm{H}$ & -3.222583000 & -1.840590000 & -1.707477000 \\
$\mathrm{H}$ & -4.610855000 & -1.840767000 & -0.636826000 \\
$\mathrm{H}$ & -2.506661000 & -2.628294000 & 0.400784000 \\
$\mathrm{~N}$ & 1.719766000 & 0.211894000 & -1.046671000 \\
$\mathrm{~N}$ & -3.220574000 & -1.132306000 & 1.677341000 \\
$\mathrm{C}$ & -6.497400000 & 0.492770000 & -0.186854000 \\
$\mathrm{C}$ & -3.916829000 & 0.124292000 & -1.244145000 \\
$\mathrm{C}$ & 2.564058000 & 0.106136000 & 1.330356000 \\
$\mathrm{C}$ & 5.702616000 & -1.556294000 & -0.853276000 \\
$\mathrm{C}$ & -1.368328000 & -0.886784000 & 0.147506000 \\
$\mathrm{C}$ & 1.953803000 & 0.977126000 & 0.222153000 \\
$\mathrm{C}$ & -2.731638000 & -1.557664000 & 0.356182000 \\
$\mathrm{C}$ & 3.988524000 & -0.391491000 & 1.070366000 \\
$\mathrm{C}$ & -3.666655000 & -1.332353000 & -0.846957000 \\
$\mathrm{C}$ & 2.796389000 & 2.193872000 & -0.145528000 \\
$\mathrm{~S}$ & 4.010570000 & -1.738049000 & -0.191920000 \\
$\mathrm{~S}$ & -4.808924000 & 1.169607000 & -0.024532000 \\
$\mathrm{O}$ & 3.092856000 & 2.471140000 & -1.280586000 \\
$\mathrm{O}$ & 3.131600000 & 2.902809000 & 0.932594000 \\
$\mathrm{O}$ & -0.735402000 & -0.940750000 & -0.896104000 \\
$\mathrm{O}$ & -0.914952000 & -0.250200000 & 1.212282000
\end{tabular}


TABLE S7: Geometry for MM-B4.

\begin{tabular}{lrrr} 
Element & $\mathrm{X}$ & $\mathrm{Y}$ & $\mathrm{Z}$ \\
\hline $\mathrm{H}$ & 3.636326000 & 3.674403000 & 0.801127000 \\
$\mathrm{H}$ & 2.638663000 & 0.616459000 & 2.265739000 \\
$\mathrm{H}$ & 1.000345000 & 1.303697000 & 0.673125000 \\
$\mathrm{H}$ & 4.663300000 & 0.424766000 & 0.679587000 \\
$\mathrm{H}$ & 4.496782000 & -0.839176000 & 1.897600000 \\
$\mathrm{H}$ & 1.957239000 & -0.799364000 & 1.498704000 \\
$\mathrm{H}$ & 2.456347000 & -0.432690000 & -1.180510000 \\
$\mathrm{H}$ & 1.729969000 & 0.949796000 & -1.778677000 \\
$\mathrm{H}$ & 6.464631000 & -1.674390000 & -0.260275000 \\
$\mathrm{H}$ & 5.815222000 & -2.171062000 & -1.834867000 \\
$\mathrm{H}$ & 5.839243000 & -0.444541000 & -1.403826000 \\
$\mathrm{H}$ & 0.767827000 & -0.221744000 & -0.982296000 \\
$\mathrm{~N}$ & 1.701471000 & 0.269031000 & -1.012711000 \\
$\mathrm{C}$ & 2.617667000 & 0.055646000 & 1.329578000 \\
$\mathrm{C}$ & 5.713726000 & -1.456400000 & -1.018940000 \\
$\mathrm{C}$ & 1.966531000 & 0.973817000 & 0.284460000 \\
$\mathrm{C}$ & 4.039611000 & -0.410979000 & 1.005550000 \\
$\mathrm{C}$ & 2.785215000 & 2.216752000 & -0.046986000 \\
$\mathrm{~S}$ & 4.040941000 & -1.689456000 & -0.325540000 \\
$\mathrm{O}$ & 3.038232000 & 2.557281000 & -1.175320000 \\
$\mathrm{O}$ & 3.152200000 & 2.871551000 & 1.054969000 \\
$\mathrm{H}$ & -6.389938000 & 2.539388000 & 0.016041000 \\
$\mathrm{~S}$ & -5.444223000 & 0.357042000 & -0.033432000 \\
$\mathrm{H}$ & -4.663363000 & 2.638222000 & -0.393242000 \\
$\mathrm{C}$ & -5.590107000 & 2.092071000 & -0.573236000 \\
$\mathrm{C}$ & -4.088289000 & -0.202855000 & -1.141871000 \\
$\mathrm{H}$ & -3.276329000 & 0.529955000 & -1.108450000 \\
$\mathrm{H}$ & -4.470863000 & -0.225244000 & -2.163531000 \\
$\mathrm{H}$ & -5.858976000 & 2.139307000 & -1.628924000 \\
$\mathrm{H}$ & -3.986325000 & -0.544178000 & 1.620919000 \\
$\mathrm{O}$ & -0.976695000 & -0.131879000 & 1.237303000 \\
$\mathrm{C}$ & -3.587803000 & -1.597722000 & -0.754563000 \\
$\mathrm{H}$ & -1.721470000 & -0.276233000 & 1.908069000 \\
$\mathrm{~N}$ & -3.196885000 & -1.177408000 & 1.756198000 \\
$\mathrm{C}$ & -1.356482000 & -0.885480000 & 0.221423000 \\
$\mathrm{O}$ & -0.703787000 & -0.968571000 & -0.807977000 \\
$\mathrm{H}$ & -4.435333000 & -2.266216000 & -0.581361000 \\
$\mathrm{C}$ & -2.660294000 & -1.656473000 & 0.472098000 \\
$\mathrm{H}$ & -3.029128000 & -2.008267000 & -1.598621000 \\
$\mathrm{H}$ & -3.487943000 & -1.935241000 & 2.361650000 \\
$\mathrm{H}$ & -2.344068000 & -2.697681000 & 0.594280000
\end{tabular}


TABLE S8: Geometry for MM-Z1.

\begin{tabular}{lrrr} 
Element & $\mathrm{X}$ & $\mathrm{Y}$ & $\mathrm{Z}$ \\
\hline $\mathrm{O}$ & -0.021004000 & 1.966472000 & -0.719614000 \\
$\mathrm{H}$ & 0.773412000 & -0.834507000 & -0.580935000 \\
$\mathrm{H}$ & 6.569770000 & -1.527510000 & 0.368462000 \\
$\mathrm{H}$ & 5.923867000 & -2.624162000 & -0.867873000 \\
$\mathrm{~N}$ & 1.597465000 & -0.151504000 & -0.807948000 \\
$\mathrm{C}$ & 5.657659000 & -1.919037000 & -0.081274000 \\
$\mathrm{H}$ & 1.332764000 & 0.370548000 & -1.646765000 \\
$\mathrm{C}$ & 0.702478000 & 1.838202000 & 0.238381000 \\
$\mathrm{H}$ & 2.470577000 & -0.676225000 & -0.994066000 \\
$\mathrm{~S}$ & 4.725081000 & -0.570003000 & -0.880879000 \\
$\mathrm{H}$ & -0.023605000 & 3.273344000 & 1.221415000 \\
$\mathrm{C}$ & 1.813017000 & 0.801015000 & 0.321298000 \\
$\mathrm{H}$ & 5.048945000 & -2.424637000 & 0.668325000 \\
$\mathrm{O}$ & 0.675282000 & 2.609332000 & 1.325782000 \\
$\mathrm{H}$ & 5.260300000 & 1.064711000 & 0.820436000 \\
$\mathrm{H}$ & 1.668123000 & 0.242619000 & 1.246775000 \\
$\mathrm{C}$ & 4.362296000 & 0.495473000 & 0.578395000 \\
$\mathrm{C}$ & 3.203360000 & 1.457539000 & 0.299471000 \\
$\mathrm{H}$ & 3.201865000 & 2.223011000 & 1.078058000 \\
$\mathrm{H}$ & 4.126795000 & -0.147922000 & 1.430155000 \\
$\mathrm{H}$ & 3.357557000 & 1.980088000 & -0.650931000 \\
$\mathrm{H}$ & -2.610110000 & 1.959619000 & -0.697335000 \\
$\mathrm{H}$ & -4.169249000 & 2.823618000 & -0.575199000 \\
$\mathrm{C}$ & -3.642799000 & 1.992354000 & -1.043563000 \\
$\mathrm{~S}$ & -4.541446000 & 0.470693000 & -0.574051000 \\
$\mathrm{H}$ & -3.855142000 & -1.098389000 & -2.287029000 \\
$\mathrm{C}$ & -3.441668000 & -0.818251000 & -1.318528000 \\
$\mathrm{H}$ & -2.463500000 & -0.366121000 & -1.484597000 \\
$\mathrm{H}$ & -3.673469000 & 2.119087000 & -2.125137000 \\
$\mathrm{C}$ & -3.313660000 & -2.058221000 & -0.431653000 \\
$\mathrm{H}$ & -3.827335000 & -0.282314000 & 1.323180000 \\
$\mathrm{H}$ & -4.300728000 & -2.474978000 & -0.205885000 \\
$\mathrm{H}$ & -2.775494000 & -2.821296000 & -0.997375000 \\
$\mathrm{~N}$ & -3.233882000 & -0.963395000 & 1.857134000 \\
$\mathrm{C}$ & -1.131818000 & -1.204020000 & 0.660090000 \\
$\mathrm{O}$ & -0.441333000 & -1.682838000 & -0.272304000 \\
$\mathrm{O}$ & -0.838721000 & -0.271684000 & 1.438069000 \\
$\mathrm{C}$ & -2.524272000 & -1.858835000 & 0.864857000 \\
$\mathrm{H}$ & -2.455685000 & -0.425522000 & 2.295752000 \\
$\mathrm{H}$ & -3.795937000 & -1.464435000 & 2.542741000 \\
$\mathrm{H}$ & -2.368319000 & -2.822926000 & 1.352091000
\end{tabular}


TABLE S9: Geometry for MM-Z2.

\begin{tabular}{lrrr} 
Element & $\mathrm{X}$ & $\mathrm{Y}$ & $\mathrm{Z}$ \\
\hline $\mathrm{H}$ & 3.579104000 & 3.687977000 & 0.290608000 \\
$\mathrm{H}$ & 2.820614000 & 0.810044000 & 2.192136000 \\
$\mathrm{H}$ & 1.034080000 & 1.230195000 & 0.668168000 \\
$\mathrm{H}$ & 4.722334000 & 0.485801000 & 0.472026000 \\
$\mathrm{H}$ & 4.712887000 & -0.600145000 & 1.861349000 \\
$\mathrm{H}$ & 2.145281000 & -0.711648000 & 1.655767000 \\
$\mathrm{H}$ & 2.392768000 & -0.671734000 & -1.075853000 \\
$\mathrm{H}$ & 1.586462000 & 0.609628000 & -1.768328000 \\
$\mathrm{H}$ & 6.551118000 & -1.680362000 & -0.263650000 \\
$\mathrm{H}$ & 5.830627000 & -2.413105000 & -1.709850000 \\
$\mathrm{H}$ & 5.817502000 & -0.643498000 & -1.526689000 \\
$\mathrm{H}$ & 0.699435000 & -0.471705000 & -0.752300000 \\
$\mathrm{H}$ & -2.955099000 & 0.152159000 & -1.530554000 \\
$\mathrm{H}$ & -6.844680000 & -0.134362000 & -1.575553000 \\
$\mathrm{H}$ & -6.571545000 & -0.814342000 & 0.054434000 \\
$\mathrm{H}$ & -2.512517000 & 0.370976000 & 2.120928000 \\
$\mathrm{H}$ & -4.029452000 & 0.314930000 & 1.359873000 \\
$\mathrm{H}$ & -4.350226000 & -0.714705000 & -2.150883000 \\
$\mathrm{H}$ & -7.299163000 & 0.794370000 & -0.133404000 \\
$\mathrm{H}$ & -3.721284000 & -0.706085000 & 2.677032000 \\
$\mathrm{H}$ & -3.010891000 & -2.290236000 & -0.895870000 \\
$\mathrm{H}$ & -4.478981000 & -2.024208000 & 0.024577000 \\
$\mathrm{H}$ & -2.444990000 & -2.137587000 & 1.432758000 \\
$\mathrm{~N}$ & 1.641084000 & 0.028138000 & -0.927037000 \\
$\mathrm{~N}$ & -3.309691000 & -0.256898000 & 1.861419000 \\
$\mathrm{C}$ & -6.572059000 & 0.095540000 & -0.545677000 \\
$\mathrm{C}$ & -3.871352000 & -0.362339000 & -1.236252000 \\
$\mathrm{C}$ & 2.753821000 & 0.138535000 & 1.334360000 \\
$\mathrm{C}$ & 5.749464000 & -1.593557000 & -0.996633000 \\
$\mathrm{C}$ & -1.306101000 & -0.566227000 & 0.511325000 \\
$\mathrm{C}$ & 1.986177000 & 0.896901000 & 0.242344000 \\
$\mathrm{C}$ & -2.667114000 & -1.219993000 & 0.885233000 \\
$\mathrm{C}$ & 4.166901000 & -0.318301000 & 0.960711000 \\
$\mathrm{C}$ & -3.557026000 & -1.543117000 & -0.315649000 \\
$\mathrm{C}$ & 2.715191000 & 2.116984000 & -0.298305000 \\
$\mathrm{~S}$ & 4.128061000 & -1.769528000 & -0.177344000 \\
$\mathrm{~S}$ & -4.953169000 & 0.943329000 & -0.512866000 \\
$\mathrm{O}$ & 2.844022000 & 2.348071000 & -1.475364000 \\
$\mathrm{O}$ & 3.162185000 & 2.904747000 & 0.684466000 \\
$\mathrm{O}$ & -0.679067000 & -1.119567000 & -0.421236000 \\
$\mathrm{O}$ & -0.974494000 & 0.430697000 & 1.190536000
\end{tabular}


TABLE S10: Geometry for MM-Z3.

\begin{tabular}{lrrr} 
Element & $\mathrm{X}$ & $\mathrm{Y}$ & $\mathrm{Z}$ \\
\hline $\mathrm{O}$ & 3.005969000 & 2.842683000 & 1.010935000 \\
$\mathrm{H}$ & 1.488972000 & 0.790977000 & -1.687035000 \\
$\mathrm{H}$ & 5.778041000 & -0.349635000 & -1.575227000 \\
$\mathrm{H}$ & 5.840952000 & -2.086106000 & -1.957213000 \\
$\mathrm{~N}$ & 1.571507000 & 0.120544000 & -0.917449000 \\
$\mathrm{C}$ & 5.734799000 & -1.355081000 & -1.156433000 \\
$\mathrm{H}$ & 2.347877000 & -0.529118000 & -1.146241000 \\
$\mathrm{C}$ & 2.575982000 & 2.160488000 & -0.054978000 \\
$\mathrm{H}$ & 0.650332000 & -0.432134000 & -0.795187000 \\
$\mathrm{~S}$ & 4.116438000 & -1.667069000 & -0.372555000 \\
$\mathrm{C}$ & 1.890418000 & 0.862747000 & 0.342723000 \\
$\mathrm{H}$ & 6.536191000 & -1.502441000 & -0.432905000 \\
$\mathrm{O}$ & 2.686332000 & 2.531976000 & -1.197478000 \\
$\mathrm{H}$ & 4.639434000 & 0.518215000 & 0.529707000 \\
$\mathrm{H}$ & 0.927957000 & 1.114947000 & 0.800499000 \\
$\mathrm{C}$ & 4.111306000 & -0.353874000 & 0.922706000 \\
$\mathrm{C}$ & 2.684820000 & 0.011516000 & 1.343335000 \\
$\mathrm{H}$ & 2.105622000 & -0.889500000 & 1.565246000 \\
$\mathrm{H}$ & 4.667733000 & -0.718400000 & 1.786541000 \\
$\mathrm{H}$ & 2.731569000 & 0.583391000 & 2.271736000 \\
$\mathrm{H}$ & 3.395473000 & 3.679085000 & 0.709053000 \\
$\mathrm{H}$ & -2.913166000 & -2.418779000 & -1.085869000 \\
$\mathrm{O}$ & -0.685565000 & -1.174421000 & -0.513261000 \\
$\mathrm{H}$ & -4.355465000 & -0.861299000 & -2.181251000 \\
$\mathrm{C}$ & -3.524364000 & -1.804308000 & -0.422457000 \\
$\mathrm{C}$ & -3.985533000 & -0.568457000 & -1.198976000 \\
$\mathrm{H}$ & -4.384366000 & -2.415409000 & -0.130564000 \\
$\mathrm{C}$ & -1.344130000 & -0.731710000 & 0.456043000 \\
$\mathrm{H}$ & -2.343974000 & -2.456168000 & 1.259215000 \\
$\mathrm{H}$ & -3.158503000 & 0.129988000 & -1.341967000 \\
$\mathrm{C}$ & -2.644203000 & -1.515208000 & 0.795091000 \\
$\mathrm{O}$ & -1.083084000 & 0.244676000 & 1.194186000 \\
$\mathrm{H}$ & -5.269361000 & 2.041164000 & -2.000230000 \\
$\mathrm{~S}$ & -5.344596000 & 0.309678000 & -0.306276000 \\
$\mathrm{~N}$ & -3.336467000 & -0.687779000 & 1.857530000 \\
$\mathrm{C}$ & -5.112753000 & 2.012533000 & -0.922388000 \\
$\mathrm{H}$ & -3.686424000 & -1.233469000 & 2.642794000 \\
$\mathrm{H}$ & -4.120443000 & 2.386779000 & -0.670633000 \\
$\mathrm{H}$ & -4.122269000 & -0.146412000 & 1.424601000 \\
$\mathrm{H}$ & -2.588949000 & -0.016538000 & 2.150143000 \\
$\mathrm{H}$ & -5.869237000 & 2.628564000 & -0.437530000
\end{tabular}


TABLE S11: Geometry for MM-Z4.

\begin{tabular}{lrrr} 
Element & $\mathrm{X}$ & $\mathrm{Y}$ & $\mathrm{Z}$ \\
\hline $\mathrm{H}$ & 4.831972000 & -2.489713000 & -1.347699000 \\
$\mathrm{H}$ & 2.220043000 & -0.297325000 & -2.270875000 \\
$\mathrm{H}$ & 1.434035000 & -1.754860000 & -0.564415000 \\
$\mathrm{H}$ & 4.141709000 & 0.835524000 & -0.966883000 \\
$\mathrm{H}$ & 3.165601000 & 1.875831000 & -2.003840000 \\
$\mathrm{H}$ & 1.086928000 & 0.549133000 & -1.245283000 \\
$\mathrm{H}$ & 2.187694000 & 0.376664000 & 1.282641000 \\
$\mathrm{H}$ & 2.331107000 & -1.206412000 & 1.781427000 \\
$\mathrm{H}$ & 4.766033000 & 3.589225000 & -0.143769000 \\
$\mathrm{H}$ & 4.291599000 & 3.665575000 & 1.564254000 \\
$\mathrm{H}$ & 5.109154000 & 2.210820000 & 0.947212000 \\
$\mathrm{H}$ & 0.786904000 & -0.629181000 & 1.282752000 \\
$\mathrm{H}$ & -2.380971000 & 0.377738000 & -1.387771000 \\
$\mathrm{H}$ & -6.104036000 & 1.528274000 & -1.578042000 \\
$\mathrm{H}$ & -6.265307000 & 0.405287000 & -0.197825000 \\
$\mathrm{H}$ & -2.412333000 & -0.529533000 & 2.273658000 \\
$\mathrm{H}$ & -3.691654000 & 0.187753000 & 1.424400000 \\
$\mathrm{H}$ & -3.908457000 & 0.251440000 & -2.244714000 \\
$\mathrm{H}$ & -6.379396000 & 2.158936000 & 0.057738000 \\
$\mathrm{H}$ & -3.983564000 & -1.189328000 & 2.366577000 \\
$\mathrm{H}$ & -3.308390000 & -1.954245000 & -1.440237000 \\
$\mathrm{H}$ & -4.714157000 & -1.436214000 & -0.529497000 \\
$\mathrm{H}$ & -3.005357000 & -2.598395000 & 0.847602000 \\
$\mathrm{~N}$ & 1.846931000 & -0.590692000 & 1.121375000 \\
$\mathrm{~N}$ & -3.303458000 & -0.722460000 & 1.769237000 \\
$\mathrm{C}$ & -5.889427000 & 1.376223000 & -0.520417000 \\
$\mathrm{C}$ & -3.444381000 & 0.170045000 & -1.260841000 \\
$\mathrm{C}$ & 2.101420000 & 0.142867000 & -1.279305000 \\
$\mathrm{C}$ & 4.405644000 & 3.007019000 & 0.703927000 \\
$\mathrm{C}$ & -1.329170000 & -1.326562000 & 0.440100000 \\
$\mathrm{C}$ & 2.185871000 & -1.014592000 & -0.275592000 \\
$\mathrm{C}$ & -2.861713000 & -1.550458000 & 0.577921000 \\
$\mathrm{C}$ & 3.141973000 & 1.252783000 & -1.109447000 \\
$\mathrm{C}$ & -3.650925000 & -1.235162000 & -0.692794000 \\
$\mathrm{C}$ & 3.542233000 & -1.699047000 & -0.219752000 \\
$\mathrm{~S}$ & 2.751858000 & 2.326817000 & 0.338800000 \\
$\mathrm{~S}$ & -4.093695000 & 1.551092000 & -0.226586000 \\
$\mathrm{O}$ & 4.120119000 & -1.946158000 & 0.810371000 \\
$\mathrm{O}$ & 3.990641000 & -2.013841000 & -1.438050000 \\
$\mathrm{O}$ & -0.785667000 & -1.730454000 & -0.584613000 \\
$\mathrm{O}$ & -0.792997000 & -0.749566000 & 1.447303000
\end{tabular}


TABLE S12: Geometry for MM-Z5.

\begin{tabular}{lrrr} 
Element & $\mathrm{X}$ & $\mathrm{Y}$ & $\mathrm{Z}$ \\
\hline $\mathrm{H}$ & 2.672509000 & 0.618725000 & 2.243100000 \\
$\mathrm{H}$ & 1.122462000 & 1.417001000 & 0.643944000 \\
$\mathrm{H}$ & 4.694769000 & 0.200038000 & 0.795376000 \\
$\mathrm{H}$ & 4.335979000 & -1.086807000 & 1.946153000 \\
$\mathrm{H}$ & 1.860338000 & -0.755446000 & 1.520307000 \\
$\mathrm{H}$ & 2.343663000 & -0.473881000 & -1.235852000 \\
$\mathrm{H}$ & 1.440494000 & 0.838115000 & -1.751058000 \\
$\mathrm{H}$ & 6.327988000 & -2.065891000 & -0.085343000 \\
$\mathrm{H}$ & 5.754488000 & -2.430256000 & -1.724561000 \\
$\mathrm{H}$ & 5.934310000 & -0.734488000 & -1.214653000 \\
$\mathrm{H}$ & 0.685907000 & -0.333379000 & -0.745592000 \\
$\mathrm{H}$ & -2.977148000 & -0.007674000 & -1.568820000 \\
$\mathrm{H}$ & -6.852828000 & -0.450685000 & -1.516782000 \\
$\mathrm{H}$ & -6.535990000 & -0.883277000 & 0.188022000 \\
$\mathrm{H}$ & -2.516835000 & 0.742040000 & 2.015792000 \\
$\mathrm{H}$ & -4.033097000 & 0.521399000 & 1.284096000 \\
$\mathrm{H}$ & -4.340428000 & -1.008437000 & -2.040809000 \\
$\mathrm{H}$ & -7.331408000 & 0.652908000 & -0.212102000 \\
$\mathrm{H}$ & -3.678208000 & -0.293739000 & 2.726796000 \\
$\mathrm{H}$ & -2.925497000 & -2.335149000 & -0.594978000 \\
$\mathrm{H}$ & -4.396270000 & -2.001404000 & 0.298514000 \\
$\mathrm{H}$ & -2.349494000 & -1.832402000 & 1.681023000 \\
$\mathrm{~N}$ & 1.602137000 & 0.200743000 & -0.970951000 \\
$\mathrm{~N}$ & -3.288354000 & 0.053401000 & 1.852704000 \\
$\mathrm{C}$ & -6.580095000 & -0.067620000 & -0.533593000 \\
$\mathrm{C}$ & -3.868955000 & -0.511170000 & -1.192003000 \\
$\mathrm{C}$ & 2.599493000 & 0.027281000 & 1.328559000 \\
$\mathrm{C}$ & 5.666537000 & -1.738958000 & -0.887146000 \\
$\mathrm{C}$ & -1.283444000 & -0.362677000 & 0.534215000 \\
$\mathrm{C}$ & 2.035969000 & 0.959883000 & 0.249012000 \\
$\mathrm{C}$ & -2.613583000 & -1.010799000 & 1.012857000 \\
$\mathrm{C}$ & 3.976312000 & -0.580182000 & 1.050027000 \\
$\mathrm{C}$ & -3.498082000 & -1.536178000 & -0.118586000 \\
$\mathrm{C}$ & 2.984215000 & 2.096429000 & -0.100759000 \\
$\mathrm{~S}$ & 3.929725000 & -1.803642000 & -0.330110000 \\
$\mathrm{~S}$ & -4.997404000 & 0.839835000 & -0.644683000 \\
$\mathrm{O}$ & 2.880672000 & 2.460241000 & -1.400926000 \\
$\mathrm{O}$ & 3.704375000 & 2.633505000 & 0.692051000 \\
$\mathrm{O}$ & -0.640608000 & -1.014594000 & -0.322313000 \\
$\mathrm{O}$ & -0.984175000 & 0.728877000 & 1.065327000 \\
$\mathrm{H}$ & 3.458378000 & 3.224307000 & -1.556769000
\end{tabular}


TABLE S13: Geometry for MW-A1.

\begin{tabular}{lrrr} 
Element & X & $\mathrm{Y}$ & $\mathrm{Z}$ \\
\hline $\mathrm{H}$ & 1.157563000 & -3.664098000 & -1.593310000 \\
$\mathrm{H}$ & 1.021624000 & -4.647468000 & 0.681360000 \\
$\mathrm{H}$ & 2.788919000 & 1.010463000 & -2.303250000 \\
$\mathrm{H}$ & 1.689493000 & -3.343138000 & 2.667180000 \\
$\mathrm{H}$ & 2.516101000 & -1.029517000 & 2.449230000 \\
$\mathrm{H}$ & 0.274788000 & 2.389101000 & 2.116520000 \\
$\mathrm{H}$ & 1.336269000 & 1.217302000 & 2.522210000 \\
$\mathrm{~N}$ & 0.939348000 & 1.720351000 & 1.733820000 \\
$\mathrm{O}$ & 0.302577000 & 2.856831000 & -0.656960000 \\
$\mathrm{C}$ & 1.439352000 & -3.086888000 & -0.720810000 \\
$\mathrm{C}$ & 1.366833000 & -3.628958000 & 0.554450000 \\
$\mathrm{C}$ & 2.010308000 & 2.430952000 & 1.014050000 \\
$\mathrm{~N}$ & 2.054761000 & -0.971878000 & -1.951810000 \\
$\mathrm{C}$ & 1.901261000 & -1.773648000 & -0.837980000 \\
$\mathrm{C}$ & 1.411827000 & 3.071632000 & -0.229450000 \\
$\mathrm{H}$ & 1.927391000 & -1.271148000 & -2.905510000 \\
$\mathrm{C}$ & 1.745322000 & -2.882238000 & 1.688670000 \\
$\mathrm{C}$ & 2.563769000 & 0.255753000 & -1.566000000 \\
$\mathrm{C}$ & 2.290421000 & -1.000267000 & 0.290080000 \\
$\mathrm{H}$ & 2.449417000 & 3.239023000 & 1.610480000 \\
$\mathrm{C}$ & 2.205711000 & -1.578997000 & 1.567540000 \\
$\mathrm{C}$ & 2.706069000 & 0.291663000 & -0.200250000 \\
$\mathrm{C}$ & 3.154878000 & 1.460243000 & 0.623120000 \\
$\mathrm{H}$ & 3.622779000 & 1.109114000 & 1.547300000 \\
$\mathrm{O}$ & 2.283806000 & 3.905973000 & -0.813450000 \\
$\mathrm{H}$ & 1.861126000 & 4.300292000 & -1.592660000 \\
$\mathrm{H}$ & 3.913948000 & 2.039374000 & 0.095220000 \\
$\mathrm{H}$ & -4.772663000 & 2.839907000 & -1.106820000 \\
$\mathrm{H}$ & -1.380051000 & 0.913140000 & -0.376220000 \\
$\mathrm{C}$ & -4.811392000 & 1.806777000 & -0.763450000 \\
$\mathrm{~N}$ & -0.631341000 & 0.242910000 & -0.124230000 \\
$\mathrm{H}$ & -5.756882000 & 1.637536000 & -0.248780000 \\
$\mathrm{~S}$ & -3.416772000 & 1.587438000 & 0.391600000 \\
$\mathrm{H}$ & -0.075060000 & 0.064391000 & -0.963430000 \\
$\mathrm{H}$ & -4.714841000 & 1.137517000 & -1.618310000 \\
$\mathrm{C}$ & -1.214579000 & -1.023660000 & 0.401360000 \\
$\mathrm{H}$ & -1.952350000 & -0.143411000 & 2.220710000 \\
$\mathrm{O}$ & -1.437019000 & -1.549581000 & -1.939970000 \\
$\mathrm{H}$ & -0.407889000 & -1.566720000 & 0.898610000 \\
$\mathrm{C}$ & -2.341330000 & -0.775411000 & 1.416350000 \\
$\mathrm{C}$ & -3.634320000 & -0.183022000 & 0.849080000 \\
$\mathrm{C}$ & -1.640859000 & -1.862791000 & -0.794110000 \\
$\mathrm{H}$ & -3.957390000 & -0.732533000 & -0.038250000 \\
$\mathrm{H}$ & -4.427000000 & -0.257793000 & 1.594290000 \\
$\mathrm{H}$ & -2.572629000 & -1.741242000 & 1.868990000 \\
$\mathrm{H}$ & -2.253608000 & -2.987061000 & -0.407770000 \\
$\mathrm{H}$ & -2.483077000 & -3.503471000 & -1.196720000 \\
& -0.016301000 & 0.728561000 & 0.590440000
\end{tabular}


TABLE S14: Geometry for MW-A2.

\begin{tabular}{lrrr} 
Element & $\mathrm{X}$ & $\mathrm{Y}$ & $\mathrm{Z}$ \\
\hline $\mathrm{H}$ & -0.190794000 & 3.603803000 & -2.205346000 \\
$\mathrm{H}$ & 1.247503000 & 4.249534000 & -0.292023000 \\
$\mathrm{H}$ & -4.008596000 & 0.417480000 & -1.611259000 \\
$\mathrm{H}$ & 0.777030000 & 3.420390000 & 1.984029000 \\
$\mathrm{H}$ & -1.097435000 & 1.873912000 & 2.410939000 \\
$\mathrm{H}$ & -0.771330000 & -2.022262000 & 2.022597000 \\
$\mathrm{H}$ & -1.235107000 & -0.622724000 & 2.723385000 \\
$\mathrm{~N}$ & -1.236424000 & -1.134510000 & 1.846014000 \\
$\mathrm{O}$ & -1.575258000 & -2.285967000 & -0.578893000 \\
$\mathrm{C}$ & -0.383233000 & 3.214833000 & -1.212272000 \\
$\mathrm{C}$ & 0.413852000 & 3.576843000 & -0.134625000 \\
$\mathrm{C}$ & -2.619121000 & -1.344246000 & 1.397083000 \\
$\mathrm{~N}$ & -2.377789000 & 1.790110000 & -1.824272000 \\
$\mathrm{C}$ & -1.449162000 & 2.342686000 & -0.964829000 \\
$\mathrm{C}$ & -2.578011000 & -2.065295000 & 0.060059000 \\
$\mathrm{H}$ & -2.507276000 & 2.051377000 & -2.788313000 \\
$\mathrm{C}$ & 0.148226000 & 3.097090000 & 1.163729000 \\
$\mathrm{C}$ & -3.203889000 & 0.938714000 & -1.116789000 \\
$\mathrm{C}$ & -1.713307000 & 1.816519000 & 0.331548000 \\
$\mathrm{H}$ & -3.190283000 & -1.979700000 & 2.085178000 \\
$\mathrm{C}$ & -0.904366000 & 2.227804000 & 1.405174000 \\
$\mathrm{C}$ & -2.820549000 & 0.901991000 & 0.201564000 \\
$\mathrm{C}$ & -3.381203000 & 0.001803000 & 1.259196000 \\
$\mathrm{H}$ & -3.364401000 & 0.494514000 & 2.236010000 \\
$\mathrm{O}$ & -3.803559000 & -2.431447000 & -0.339441000 \\
$\mathrm{H}$ & -3.725496000 & -2.900650000 & -1.184863000 \\
$\mathrm{H}$ & -4.425770000 & -0.230279000 & 1.047028000 \\
$\mathrm{H}$ & 4.839143000 & 1.140513000 & 2.285516000 \\
$\mathrm{H}$ & 1.413516000 & 0.269484000 & 0.548369000 \\
$\mathrm{C}$ & 4.816077000 & 0.395860000 & 1.490560000 \\
$\mathrm{~N}$ & 0.636112000 & -0.124047000 & -0.012170000 \\
$\mathrm{H}$ & 5.797791000 & 0.349418000 & 1.019896000 \\
$\mathrm{~S}$ & 3.564531000 & 0.953398000 & 0.285069000 \\
$\mathrm{H}$ & -0.099348000 & -0.529278000 & 0.648841000 \\
$\mathrm{H}$ & 4.544475000 & -0.573392000 & 1.908614000 \\
$\mathrm{C}$ & 1.176189000 & -1.116285000 & -0.982814000 \\
$\mathrm{H}$ & 2.101783000 & 0.385545000 & -2.218985000 \\
$\mathrm{O}$ & 1.458123000 & -2.482286000 & 0.990193000 \\
$\mathrm{H}$ & 0.371088000 & -1.362161000 & -1.674173000 \\
$\mathrm{C}$ & 2.386012000 & -0.567103000 & -1.760692000 \\
$\mathrm{C}$ & 3.677905000 & -0.408824000 & -0.953619000 \\
$\mathrm{C}$ & 1.506131000 & -2.383727000 & -0.210048000 \\
$\mathrm{H}$ & 3.915765000 & -1.331387000 & -0.419051000 \\
$\mathrm{H}$ & 4.505017000 & -0.194084000 & -1.630939000 \\
$\mathrm{H}$ & 2.578502000 & -1.260775000 & -2.581673000 \\
$\mathrm{H}$ & 1.875646000 & -3.362778000 & -1.041792000 \\
$\mathrm{H}$ & 2.071506000 & -4.158682000 & -0.522122000 \\
& 0.198032000 & 0.662022000 & -0.499637000
\end{tabular}


TABLE S15: Geometry for MW-A3.

\begin{tabular}{lrrr} 
Element & $\mathrm{X}$ & $\mathrm{Y}$ & $\mathrm{Z}$ \\
\hline $\mathrm{H}$ & -5.111067000 & 0.816668000 & -2.573140000 \\
$\mathrm{H}$ & -1.489541000 & 0.059695000 & -1.050722000 \\
$\mathrm{C}$ & -5.026299000 & 0.298184000 & -1.618760000 \\
$\mathrm{~N}$ & -0.674365000 & -0.122294000 & -0.442189000 \\
$\mathrm{H}$ & -5.930636000 & 0.474791000 & -1.036976000 \\
$\mathrm{~S}$ & -3.579594000 & 1.005563000 & -0.761054000 \\
$\mathrm{H}$ & 0.054770000 & -0.704397000 & -0.966617000 \\
$\mathrm{H}$ & -4.886128000 & -0.768075000 & -1.795593000 \\
$\mathrm{C}$ & -1.135374000 & -0.769254000 & 0.821052000 \\
$\mathrm{H}$ & -1.902535000 & 1.052094000 & 1.666830000 \\
$\mathrm{O}$ & -1.592116000 & -2.624614000 & -0.651034000 \\
$\mathrm{H}$ & -0.271476000 & -0.824635000 & 1.481357000 \\
$\mathrm{C}$ & -2.255464000 & 0.028324000 & 1.508974000 \\
$\mathrm{C}$ & -3.610963000 & 0.021923000 & 0.797952000 \\
$\mathrm{C}$ & -1.543529000 & -2.191108000 & 0.472446000 \\
$\mathrm{H}$ & -3.915076000 & -0.997484000 & 0.548653000 \\
$\mathrm{H}$ & -4.369844000 & 0.445535000 & 1.456567000 \\
$\mathrm{H}$ & -2.387908000 & -0.408114000 & 2.501130000 \\
$\mathrm{O}$ & -1.860296000 & -2.880143000 & 1.573001000 \\
$\mathrm{H}$ & -2.099606000 & -3.785058000 & 1.317041000 \\
$\mathrm{H}$ & -0.239781000 & 0.778864000 & -0.221059000 \\
$\mathrm{H}$ & 3.562910000 & -2.480233000 & 1.674889000 \\
$\mathrm{O}$ & 3.686181000 & -2.143033000 & 0.773735000 \\
$\mathrm{O}$ & 1.444158000 & -2.277797000 & 0.753702000 \\
$\mathrm{C}$ & 2.483854000 & -2.028606000 & 0.190274000 \\
$\mathrm{H}$ & 1.625970000 & 1.383916000 & 3.539069000 \\
$\mathrm{H}$ & 2.813741000 & 0.212202000 & 1.724509000 \\
$\mathrm{H}$ & 3.277019000 & -2.321575000 & -1.723922000 \\
$\mathrm{C}$ & 2.607791000 & -1.587132000 & -1.257689000 \\
$\mathrm{C}$ & 1.476840000 & 1.692812000 & 2.511685000 \\
$\mathrm{C}$ & 2.141050000 & 1.027675000 & 1.493141000 \\
$\mathrm{H}$ & 1.325338000 & -1.307765000 & -2.832123000 \\
$\mathrm{H}$ & 4.193882000 & -0.200162000 & -0.794225000 \\
$\mathrm{~N}$ & 1.267922000 & -1.593874000 & -1.858734000 \\
$\mathrm{C}$ & 3.296931000 & -0.200925000 & -1.416961000 \\
$\mathrm{C}$ & 0.622418000 & 2.779988000 & 2.239117000 \\
$\mathrm{C}$ & 1.950267000 & 1.444864000 & 0.163599000 \\
$\mathrm{H}$ & 0.125459000 & 3.284014000 & 3.058883000 \\
$\mathrm{C}$ & 2.424033000 & 0.979695000 & -1.119491000 \\
$\mathrm{H}$ & 3.642143000 & -0.130680000 & -2.451777000 \\
$\mathrm{C}$ & 0.424077000 & 3.231028000 & 0.942169000 \\
$\mathrm{C}$ & 1.095191000 & 2.557925000 & -0.083950000 \\
$\mathrm{C}$ & 1.874469000 & 1.813434000 & -2.061790000 \\
$\mathrm{H}$ & -0.219747000 & 4.077488000 & 0.734607000 \\
$\mathrm{~N}$ & 1.064068000 & 2.751866000 & -1.452144000 \\
$\mathrm{H}$ & 2.001444000 & 1.813414000 & -3.133470000 \\
$\mathrm{H}$ & 0.628664000 & 3.531086000 & -1.919311000 \\
& 0.866964000 & -2.528754000 & -1.842095000
\end{tabular}


TABLE S16: Geometry for MW-A4.

\begin{tabular}{lrrr} 
Element & $\mathrm{X}$ & $\mathrm{Y}$ & $\mathrm{Z}$ \\
\hline $\mathrm{O}$ & -2.840500000 & 1.556112000 & 0.897656000 \\
$\mathrm{H}$ & -5.029439000 & -2.024265000 & 1.183819000 \\
$\mathrm{H}$ & -4.476605000 & -3.611473000 & 1.767798000 \\
$\mathrm{C}$ & -4.547229000 & -2.958923000 & 0.898394000 \\
$\mathrm{H}$ & -3.742938000 & 2.471191000 & -1.060573000 \\
$\mathrm{C}$ & -2.518950000 & 1.295187000 & -0.233488000 \\
$\mathrm{H}$ & -0.690956000 & 0.505573000 & 1.347841000 \\
$\mathrm{H}$ & -5.117934000 & -3.463889000 & 0.119541000 \\
$\mathrm{O}$ & -3.067140000 & 1.828685000 & -1.329860000 \\
$\mathrm{~N}$ & -0.887962000 & -0.252109000 & 0.686416000 \\
$\mathrm{H}$ & -1.591979000 & -0.886743000 & 1.101588000 \\
$\mathrm{H}$ & -3.960827000 & -0.866890000 & -0.852590000 \\
$\mathrm{~S}$ & -2.840616000 & -2.658157000 & 0.328324000 \\
$\mathrm{C}$ & -1.425232000 & 0.299524000 & -0.592956000 \\
$\mathrm{C}$ & -3.161536000 & -1.562969000 & -1.117655000 \\
$\mathrm{H}$ & -0.615842000 & 0.857959000 & -1.067530000 \\
$\mathrm{C}$ & -1.901392000 & -0.807233000 & -1.547455000 \\
$\mathrm{H}$ & -3.515373000 & -2.178378000 & -1.945500000 \\
$\mathrm{H}$ & -2.099602000 & -0.327529000 & -2.507759000 \\
$\mathrm{H}$ & -1.075188000 & -1.504656000 & -1.714669000 \\
$\mathrm{H}$ & 0.004868000 & -0.827273000 & 0.531548000 \\
$\mathrm{H}$ & 1.889488000 & 0.600576000 & 3.265944000 \\
$\mathrm{H}$ & 0.333397000 & 2.563088000 & 3.003776000 \\
$\mathrm{C}$ & 1.682812000 & 1.066817000 & 2.314987000 \\
$\mathrm{~N}$ & 0.725327000 & 2.070899000 & 2.215471000 \\
$\mathrm{~N}$ & 1.365111000 & -1.900535000 & 0.376254000 \\
$\mathrm{O}$ & 3.469168000 & -3.570964000 & -0.278534000 \\
$\mathrm{H}$ & 3.559747000 & -0.751563000 & 1.636737000 \\
$\mathrm{C}$ & 2.225103000 & 0.818053000 & 1.081977000 \\
$\mathrm{C}$ & 0.697380000 & 2.536586000 & 0.908394000 \\
$\mathrm{C}$ & 3.200664000 & -0.257929000 & 0.727834000 \\
$\mathrm{C}$ & 2.584057000 & -1.322240000 & -0.210291000 \\
$\mathrm{C}$ & 3.605486000 & -2.405532000 & -0.547043000 \\
$\mathrm{H}$ & -0.774505000 & 4.127606000 & 0.905276000 \\
$\mathrm{C}$ & -0.080826000 & 3.535582000 & 0.320566000 \\
$\mathrm{C}$ & 1.615589000 & 1.751275000 & 0.163041000 \\
$\mathrm{O}$ & 4.669915000 & -1.893465000 & -1.188556000 \\
$\mathrm{H}$ & 2.309611000 & -0.839112000 & -1.152322000 \\
$\mathrm{H}$ & 4.077803000 & 0.160307000 & 0.229592000 \\
$\mathrm{H}$ & 5.290613000 & -2.613997000 & -1.382125000 \\
$\mathrm{C}$ & 0.072303000 & 3.744264000 & -1.043273000 \\
$\mathrm{C}$ & 1.751452000 & 1.989040000 & -1.215652000 \\
$\mathrm{H}$ & -0.508384000 & 4.517175000 & -1.531321000 \\
$\mathrm{C}$ & 0.978477000 & 2.979772000 & -1.804084000 \\
$\mathrm{H}$ & 2.462783000 & 1.427897000 & -1.810216000 \\
$\mathrm{H}$ & 1.080959000 & 3.181958000 & -2.863089000 \\
$\mathrm{H}$ & 1.584543000 & -2.243165000 & 1.311294000 \\
& 1.117810000 & -2.742264000 & -0.142657000
\end{tabular}


TABLE S17: Geometry for MW-A5.

\begin{tabular}{lrrr} 
Element & $\mathrm{X}$ & $\mathrm{Y}$ & $\mathrm{Z}$ \\
\hline $\mathrm{O}$ & 4.903765000 & -2.335233000 & -0.745142000 \\
$\mathrm{O}$ & 4.033560000 & -2.580178000 & 1.321058000 \\
$\mathrm{~N}$ & 0.548623000 & 2.036829000 & -2.252501000 \\
$\mathrm{~N}$ & 1.652066000 & -1.355111000 & 0.587181000 \\
$\mathrm{C}$ & 2.158884000 & 1.039658000 & -1.019818000 \\
$\mathrm{C}$ & 3.268116000 & 0.128241000 & -0.591878000 \\
$\mathrm{C}$ & 1.399190000 & 1.919881000 & -0.163420000 \\
$\mathrm{C}$ & 2.796741000 & -1.323401000 & -0.333191000 \\
$\mathrm{C}$ & 0.416493000 & 2.552593000 & -0.970970000 \\
$\mathrm{C}$ & 1.631699000 & 1.164532000 & -2.278842000 \\
$\mathrm{C}$ & 1.450109000 & 2.224696000 & 1.206891000 \\
$\mathrm{C}$ & -0.496839000 & 3.473930000 & -0.453186000 \\
$\mathrm{C}$ & 3.962108000 & -2.155409000 & 0.197362000 \\
$\mathrm{C}$ & 0.545111000 & 3.139446000 & 1.725957000 \\
$\mathrm{C}$ & -0.417023000 & 3.759127000 & 0.903647000 \\
$\mathrm{H}$ & 4.057012000 & 0.095076000 & -1.344529000 \\
$\mathrm{H}$ & 3.727907000 & 0.517262000 & 0.322224000 \\
$\mathrm{H}$ & 2.480659000 & -1.758466000 & -1.285593000 \\
$\mathrm{H}$ & 1.932143000 & 0.697222000 & -3.203934000 \\
$\mathrm{H}$ & 0.133536000 & 2.443554000 & -3.076857000 \\
$\mathrm{H}$ & 2.196809000 & 1.772385000 & 1.849526000 \\
$\mathrm{H}$ & -1.229699000 & 3.961767000 & -1.085314000 \\
$\mathrm{H}$ & 0.583582000 & 3.394454000 & 2.777774000 \\
$\mathrm{H}$ & 1.434501000 & -2.318455000 & 0.837258000 \\
$\mathrm{H}$ & -1.099507000 & 4.480144000 & 1.336330000 \\
$\mathrm{H}$ & 5.632316000 & -2.848387000 & -0.360637000 \\
$\mathrm{H}$ & 1.919329000 & -0.919949000 & 1.468580000 \\
$\mathrm{H}$ & -4.225402000 & -3.508562000 & -2.181695000 \\
$\mathrm{C}$ & -4.423036000 & -2.877575000 & -1.315931000 \\
$\mathrm{H}$ & -4.091968000 & -3.387458000 & -0.411417000 \\
$\mathrm{H}$ & -5.490439000 & -2.663784000 & -1.268150000 \\
$\mathrm{~S}$ & -3.484194000 & -1.333280000 & -1.566586000 \\
$\mathrm{H}$ & -1.295519000 & -1.142236000 & -0.798088000 \\
$\mathrm{~N}$ & -0.749207000 & -0.380171000 & -0.363532000 \\
$\mathrm{H}$ & 0.209389000 & -0.748969000 & -0.005723000 \\
$\mathrm{H}$ & -0.571747000 & 0.324289000 & -1.082962000 \\
$\mathrm{O}$ & -1.194641000 & -1.934137000 & 1.770549000 \\
$\mathrm{C}$ & -3.942199000 & -0.416078000 & -0.035460000 \\
$\mathrm{H}$ & -4.006251000 & -1.134444000 & 0.785293000 \\
$\mathrm{H}$ & -4.932458000 & 0.018971000 & -0.174717000 \\
$\mathrm{C}$ & -1.547536000 & 0.222134000 & 0.745199000 \\
$\mathrm{C}$ & -1.619986000 & -0.810478000 & 1.859376000 \\
$\mathrm{C}$ & -2.938883000 & 0.693129000 & 0.289211000 \\
$\mathrm{H}$ & -2.811549000 & 1.365713000 & -0.564235000 \\
$\mathrm{H}$ & -0.986433000 & 1.081608000 & 1.114211000 \\
$\mathrm{H}$ & -2.222388000 & -0.303451000 & 2.940930000 \\
$\mathrm{H}$ & -3.346239000 & 1.301830000 & 1.098905000 \\
& -2.253321000 & -0.984485000 & 3.632252000
\end{tabular}


TABLE S18: Geometry for MW-A6.

\begin{tabular}{lrrr} 
Element & $\mathrm{X}$ & $\mathrm{Y}$ & $\mathrm{Z}$ \\
\hline $\mathrm{O}$ & 4.513505000 & -2.379260000 & -1.028175000 \\
$\mathrm{O}$ & 3.697982000 & -3.169760000 & 0.918891000 \\
$\mathrm{~N}$ & 0.952240000 & 2.656659000 & -1.606056000 \\
$\mathrm{~N}$ & 1.524047000 & -1.476346000 & 0.868738000 \\
$\mathrm{C}$ & 2.219997000 & 1.179857000 & -0.464046000 \\
$\mathrm{C}$ & 3.190618000 & 0.081432000 & -0.156216000 \\
$\mathrm{C}$ & 1.401334000 & 1.907725000 & 0.473027000 \\
$\mathrm{C}$ & 2.560832000 & -1.330733000 & -0.167136000 \\
$\mathrm{C}$ & 0.610560000 & 2.821314000 & -0.278460000 \\
$\mathrm{C}$ & 1.928615000 & 1.682062000 & -1.709528000 \\
$\mathrm{C}$ & 1.243070000 & 1.865444000 & 1.869021000 \\
$\mathrm{C}$ & -0.346876000 & 3.645740000 & 0.318046000 \\
$\mathrm{C}$ & 3.641279000 & -2.401275000 & -0.005661000 \\
$\mathrm{C}$ & 0.291770000 & 2.682069000 & 2.464390000 \\
$\mathrm{C}$ & -0.501192000 & 3.558197000 & 1.694038000 \\
$\mathrm{H}$ & 4.002165000 & 0.085129000 & -0.884712000 \\
$\mathrm{H}$ & 3.652865000 & 0.250436000 & 0.822607000 \\
$\mathrm{H}$ & 2.095355000 & -1.487454000 & -1.145102000 \\
$\mathrm{H}$ & 2.351798000 & 1.429142000 & -2.669436000 \\
$\mathrm{H}$ & 0.628629000 & 3.232002000 & -2.367065000 \\
$\mathrm{H}$ & 1.855216000 & 1.210150000 & 2.478079000 \\
$\mathrm{H}$ & -0.945977000 & 4.332572000 & -0.268038000 \\
$\mathrm{H}$ & 0.163464000 & 2.661838000 & 3.539666000 \\
$\mathrm{H}$ & 1.284791000 & -2.464417000 & 0.949210000 \\
$\mathrm{H}$ & -1.236114000 & 4.181109000 & 2.187829000 \\
$\mathrm{H}$ & 5.179087000 & -3.070186000 & -0.881278000 \\
$\mathrm{H}$ & 1.939483000 & -1.253493000 & 1.772517000 \\
$\mathrm{H}$ & -1.651388000 & -0.945208000 & -2.547425000 \\
$\mathrm{H}$ & -3.031812000 & -2.755402000 & -1.810193000 \\
$\mathrm{C}$ & -1.568554000 & -1.205581000 & -1.490209000 \\
$\mathrm{H}$ & -0.684882000 & -1.844209000 & -1.396988000 \\
$\mathrm{C}$ & -2.828616000 & -1.972228000 & -1.079121000 \\
$\mathrm{O}$ & -2.759588000 & 1.473434000 & -1.992054000 \\
$\mathrm{H}$ & -0.464621000 & 0.621302000 & -1.200810000 \\
$\mathrm{H}$ & -3.696731000 & -1.309617000 & -1.047079000 \\
$\mathrm{H}$ & -3.552771000 & 2.032856000 & -1.969361000 \\
$\mathrm{C}$ & -1.303841000 & 0.108605000 & -0.732718000 \\
$\mathrm{C}$ & -2.511158000 & 1.032640000 & -0.750173000 \\
$\mathrm{~S}$ & -2.636731000 & -2.752236000 & 0.578674000 \\
$\mathrm{H}$ & -4.854738000 & -3.695732000 & 0.308267000 \\
$\mathrm{C}$ & -4.385918000 & -3.020163000 & 1.023114000 \\
$\mathrm{~N}$ & -0.935615000 & -0.133518000 & 0.691628000 \\
$\mathrm{H}$ & 0.025381000 & -0.607301000 & 0.747002000 \\
$\mathrm{O}$ & -3.160451000 & 1.309288000 & 0.223119000 \\
$\mathrm{H}$ & -4.921162000 & -2.071954000 & 1.066402000 \\
$\mathrm{H}$ & -1.634895000 & -0.761911000 & 1.124791000 \\
& -0.893200000 & 0.743552000 & 1.219530000 \\
& -4.390071000 & -3.485248000 & 2.008183000
\end{tabular}


TABLE S19: Geometry for MW-A7.

\begin{tabular}{lrrr} 
Element & $\mathrm{X}$ & $\mathrm{Y}$ & $\mathrm{Z}$ \\
\hline $\mathrm{C}$ & 1.966382000 & 1.675546000 & -0.681910000 \\
$\mathrm{C}$ & 0.865430000 & 2.688396000 & -0.725581000 \\
$\mathrm{H}$ & 1.037247000 & 3.498979000 & -0.014441000 \\
$\mathrm{H}$ & 2.786111000 & 1.653481000 & -2.745341000 \\
$\mathrm{H}$ & 4.290403000 & -0.179171000 & -1.913950000 \\
$\mathrm{C}$ & 2.748748000 & 1.274984000 & -1.735547000 \\
$\mathrm{~N}$ & 3.565746000 & 0.229742000 & -1.346095000 \\
$\mathrm{H}$ & -0.518683000 & 1.781684000 & 0.664836000 \\
$\mathrm{C}$ & 3.329722000 & -0.061098000 & -0.017337000 \\
$\mathrm{H}$ & 4.625388000 & -1.739678000 & 0.443946000 \\
$\mathrm{O}$ & -1.452869000 & 4.186143000 & 0.019316000 \\
$\mathrm{C}$ & -0.502474000 & 2.048165000 & -0.396470000 \\
$\mathrm{C}$ & 3.859432000 & -1.061973000 & 0.802522000 \\
$\mathrm{C}$ & -1.669374000 & 3.007796000 & -0.582496000 \\
$\mathrm{H}$ & -2.235200000 & 4.746435000 & -0.107201000 \\
$\mathrm{C}$ & 2.330354000 & 0.844858000 & 0.439416000 \\
$\mathrm{~N}$ & -0.731774000 & 0.810353000 & -1.160112000 \\
$\mathrm{O}$ & -2.687690000 & 2.723344000 & -1.164554000 \\
$\mathrm{C}$ & 3.375031000 & -1.152081000 & 2.101080000 \\
$\mathrm{H}$ & 0.824292000 & 3.144296000 & -1.719503000 \\
$\mathrm{C}$ & 1.880374000 & 0.743996000 & 1.767805000 \\
$\mathrm{H}$ & -1.728413000 & 0.734662000 & -1.359479000 \\
$\mathrm{C}$ & 2.398092000 & -0.255617000 & 2.581072000 \\
$\mathrm{H}$ & 1.146912000 & 1.437012000 & 2.161718000 \\
$\mathrm{H}$ & 2.064680000 & -0.336666000 & 3.608499000 \\
$\mathrm{H}$ & -0.270142000 & 0.857120000 & -2.066431000 \\
$\mathrm{H}$ & 3.770029000 & -1.911785000 & 2.764181000 \\
$\mathrm{H}$ & 0.289933000 & -3.482821000 & 0.007123000 \\
$\mathrm{O}$ & -1.489632000 & -3.872666000 & -1.903344000 \\
$\mathrm{C}$ & -0.410296000 & -2.664017000 & -0.182381000 \\
$\mathrm{H}$ & -2.037487000 & -3.799179000 & 0.553604000 \\
$\mathrm{C}$ & -0.803578000 & -2.753515000 & -1.654619000 \\
$\mathrm{C}$ & -1.591360000 & -2.830717000 & 0.784411000 \\
$\mathrm{H}$ & -1.200898000 & -2.908848000 & 1.803591000 \\
$\mathrm{H}$ & 1.245922000 & -1.417996000 & -0.441177000 \\
$\mathrm{~N}$ & 0.323848000 & -1.379886000 & -0.002718000 \\
$\mathrm{O}$ & -0.507865000 & -1.927465000 & -2.480333000 \\
$\mathrm{C}$ & -2.676450000 & -1.750493000 & 0.722872000 \\
$\mathrm{H}$ & 0.493252000 & -1.169015000 & 0.984782000 \\
$\mathrm{H}$ & -4.596401000 & 0.187539000 & 1.877859000 \\
$\mathrm{H}$ & -3.613771000 & -2.161926000 & 1.099422000 \\
$\mathrm{H}$ & -2.852128000 & -1.414179000 & -0.303448000 \\
$\mathrm{C}$ & -3.706054000 & 0.722771000 & 1.547431000 \\
$\mathrm{~S}$ & -2.226433000 & -0.319895000 & 1.781374000 \\
$\mathrm{H}$ & -3.811582000 & 1.038012000 & 0.509670000 \\
$\mathrm{H}$ & -3.571455000 & 1.602034000 & 2.177276000 \\
$\mathrm{H}$ & -1.696029000 & -3.913231000 & -2.851216000 \\
& -0.183207000 & -0.486763000 & -0.464119000
\end{tabular}


TABLE S20: Geometry for MW-B1.

\begin{tabular}{lrrr} 
Element & $\mathrm{X}$ & $\mathrm{Y}$ & $\mathrm{Z}$ \\
\hline $\mathrm{H}$ & -6.063415000 & -3.029168000 & -0.299999000 \\
$\mathrm{H}$ & -4.211587000 & -1.047579000 & 1.973794000 \\
$\mathrm{H}$ & -2.813877000 & -1.686196000 & 0.151003000 \\
$\mathrm{H}$ & -6.012836000 & 0.236424000 & 0.652277000 \\
$\mathrm{H}$ & -5.503798000 & 0.951586000 & 2.181259000 \\
$\mathrm{H}$ & -3.092181000 & 0.252191000 & 1.633623000 \\
$\mathrm{H}$ & -3.570898000 & 0.900179000 & -0.995902000 \\
$\mathrm{H}$ & -3.270863000 & -0.382515000 & -2.025398000 \\
$\mathrm{H}$ & -7.040619000 & 2.956855000 & 0.482719000 \\
$\mathrm{H}$ & -6.205173000 & 3.686529000 & -0.901990000 \\
$\mathrm{H}$ & -6.771869000 & 2.003087000 & -1.010455000 \\
$\mathrm{H}$ & -2.038970000 & 0.132197000 & -0.952887000 \\
$\mathrm{~N}$ & -3.076315000 & -0.014170000 & -1.089041000 \\
$\mathrm{C}$ & -3.979775000 & -0.261007000 & 1.253397000 \\
$\mathrm{C}$ & -6.360480000 & 2.762681000 & -0.345945000 \\
$\mathrm{C}$ & -3.607860000 & -0.966011000 & -0.059848000 \\
$\mathrm{C}$ & -5.171349000 & 0.697451000 & 1.174750000 \\
$\mathrm{C}$ & -4.763659000 & -1.720416000 & -0.707077000 \\
$\mathrm{~S}$ & -4.726648000 & 2.256743000 & 0.292780000 \\
$\mathrm{O}$ & -5.069438000 & -1.591089000 & -1.865721000 \\
$\mathrm{O}$ & -5.360578000 & -2.540670000 & 0.158992000 \\
$\mathrm{H}$ & 5.455723000 & 3.572890000 & -0.473593000 \\
$\mathrm{C}$ & 5.599725000 & 2.505758000 & -0.356284000 \\
$\mathrm{H}$ & 3.540006000 & 2.039272000 & -0.740659000 \\
$\mathrm{H}$ & 7.710247000 & 2.711670000 & 0.033130000 \\
$\mathrm{C}$ & 4.521366000 & 1.646286000 & -0.498776000 \\
$\mathrm{C}$ & 6.887806000 & 2.014466000 & -0.069804000 \\
$\mathrm{O}$ & -0.424239000 & 0.278341000 & -0.649289000 \\
$\mathrm{H}$ & 1.898062000 & 0.822372000 & 0.560717000 \\
$\mathrm{H}$ & 2.136776000 & -0.334449000 & -1.573049000 \\
$\mathrm{C}$ & 4.730083000 & 0.265760000 & -0.350185000 \\
$\mathrm{C}$ & 7.126530000 & 0.655543000 & 0.074679000 \\
$\mathrm{C}$ & 0.142427000 & -0.335362000 & 0.242007000 \\
$\mathrm{C}$ & 1.635286000 & -0.237071000 & 0.518615000 \\
$\mathrm{C}$ & 2.400766000 & -0.888986000 & -0.669258000 \\
$\mathrm{C}$ & 6.038609000 & -0.206768000 & -0.068787000 \\
$\mathrm{H}$ & 8.121179000 & 0.281405000 & 0.286381000 \\
$\mathrm{C}$ & 3.873891000 & -0.891548000 & -0.435779000 \\
$\mathrm{O}$ & -0.504379000 & -1.185064000 & 1.027913000 \\
$\mathrm{H}$ & 2.040720000 & -1.914532000 & -0.801452000 \\
$\mathrm{~N}$ & 1.904609000 & -0.935970000 & 1.781467000 \\
$\mathrm{H}$ & 2.033602000 & -0.286878000 & 2.550430000 \\
$\mathrm{~N}$ & 5.967620000 & -1.584845000 & 0.012438000 \\
$\mathrm{H}$ & 4.668639000 & -1.990514000 & -0.208429000 \\
& 2.762064000 & -1.478617000 & 1.706234000 \\
$\mathrm{H}$ & 6.749438000 & -2.200919000 & 0.162036000 \\
& 4.410668000 & -3.038588000 & -0.209886000 \\
0.209588000 & -1.503909000 & 1.658319000
\end{tabular}


TABLE S21: Geometry for MW-Z1.

\begin{tabular}{lrrr} 
Element & $\mathrm{X}$ & $\mathrm{Y}$ & $\mathrm{Z}$ \\
\hline $\mathrm{H}$ & -5.715490000 & -3.169713000 & 0.543377000 \\
$\mathrm{H}$ & -4.089104000 & -0.526012000 & 2.241970000 \\
$\mathrm{H}$ & -2.620767000 & -1.416891000 & 0.589275000 \\
$\mathrm{H}$ & -6.020923000 & 0.200393000 & 0.691680000 \\
$\mathrm{H}$ & -5.609843000 & 1.294530000 & 2.011863000 \\
$\mathrm{H}$ & -3.126344000 & 0.771124000 & 1.569789000 \\
$\mathrm{H}$ & -3.636955000 & 0.714359000 & -1.126030000 \\
$\mathrm{H}$ & -3.202071000 & -0.736978000 & -1.814846000 \\
$\mathrm{H}$ & -7.351065000 & 2.708855000 & -0.002825000 \\
$\mathrm{H}$ & -6.624914000 & 3.199456000 & -1.545407000 \\
$\mathrm{H}$ & -7.007130000 & 1.484835000 & -1.264633000 \\
$\mathrm{H}$ & -2.012123000 & 0.135393000 & -0.908446000 \\
$\mathrm{~N}$ & -3.057538000 & -0.136334000 & -0.998616000 \\
$\mathrm{C}$ & -3.949170000 & 0.085181000 & 1.348605000 \\
$\mathrm{C}$ & -6.667591000 & 2.409033000 & -0.796840000 \\
$\mathrm{C}$ & -3.497120000 & -0.866041000 & 0.231287000 \\
$\mathrm{C}$ & -5.242469000 & 0.860553000 & 1.081542000 \\
$\mathrm{C}$ & -4.551361000 & -1.877549000 & -0.188637000 \\
$\mathrm{~S}$ & -4.977344000 & 2.222223000 & -0.134166000 \\
$\mathrm{O}$ & -4.845438000 & -2.096551000 & -1.337760000 \\
$\mathrm{O}$ & -5.082804000 & -2.507317000 & 0.864629000 \\
$\mathrm{H}$ & 2.197245000 & -0.327344000 & 2.299526000 \\
$\mathrm{H}$ & 5.689736000 & 3.512689000 & 0.393721000 \\
$\mathrm{H}$ & 7.851927000 & 2.381541000 & 0.746736000 \\
$\mathrm{C}$ & 5.754212000 & 2.440780000 & 0.252961000 \\
$\mathrm{C}$ & 6.990495000 & 1.795645000 & 0.450842000 \\
$\mathrm{~N}$ & 2.014583000 & -0.888530000 & 1.469106000 \\
$\mathrm{H}$ & 1.806149000 & 0.985896000 & 0.499525000 \\
$\mathrm{C}$ & 4.628707000 & 1.729062000 & -0.130983000 \\
$\mathrm{H}$ & 3.691684000 & 2.245476000 & -0.307109000 \\
$\mathrm{C}$ & 1.585448000 & -0.056935000 & 0.283352000 \\
$\mathrm{C}$ & 7.130617000 & 0.428632000 & 0.263870000 \\
$\mathrm{O}$ & -0.421633000 & -1.185478000 & 0.868359000 \\
$\mathrm{H}$ & 2.866750000 & -1.418334000 & 1.236510000 \\
$\mathrm{C}$ & 0.063453000 & -0.280339000 & 0.148881000 \\
$\mathrm{H}$ & 8.086426000 & -0.061124000 & 0.406747000 \\
$\mathrm{C}$ & 4.736990000 & 0.340988000 & -0.319427000 \\
$\mathrm{O}$ & -0.515290000 & 0.444140000 & -0.692564000 \\
$\mathrm{C}$ & 5.995313000 & -0.285826000 & -0.122623000 \\
$\mathrm{C}$ & 3.808731000 & -0.692167000 & -0.713157000 \\
$\mathrm{C}$ & 2.346718000 & -0.526794000 & -0.977682000 \\
$\mathrm{~N}$ & 5.827702000 & -1.633465000 & -0.382436000 \\
$\mathrm{C}$ & 4.517236000 & -1.874004000 & -0.733320000 \\
$\mathrm{H}$ & 2.147916000 & 0.212501000 & -1.756131000 \\
$\mathrm{H}$ & 6.559799000 & -2.324814000 & -0.370764000 \\
$\mathrm{H}$ & 1.918522000 & -1.472560000 & -1.323097000 \\
$\mathrm{H}$ & 4.191591000 & -2.865525000 & -1.009454000 \\
$\mathrm{H}$ & 1.173326000 & -1.494673000 & 1.634974000
\end{tabular}


TABLE S22: Geometry for WM-A1.

\begin{tabular}{lrrr} 
Element & $\mathrm{X}$ & $\mathrm{Y}$ & $\mathrm{Z}$ \\
\hline $\mathrm{C}$ & 0.491103000 & -2.087140000 & -0.805574000 \\
$\mathrm{C}$ & 1.967344000 & -1.993855000 & -0.581598000 \\
$\mathrm{H}$ & 2.319619000 & -2.766087000 & 0.106232000 \\
$\mathrm{H}$ & 0.304348000 & -2.161939000 & -3.019674000 \\
$\mathrm{H}$ & -2.161055000 & -2.316722000 & -2.606406000 \\
$\mathrm{C}$ & -0.128460000 & -2.140534000 & -2.031359000 \\
$\mathrm{~N}$ & -1.495048000 & -2.186943000 & -1.862054000 \\
$\mathrm{H}$ & 2.036802000 & -0.542989000 & 1.045888000 \\
$\mathrm{C}$ & -1.791312000 & -2.177803000 & -0.512829000 \\
$\mathrm{H}$ & -3.956036000 & -2.268434000 & -0.407843000 \\
$\mathrm{O}$ & 4.520905000 & -1.256742000 & 0.825778000 \\
$\mathrm{C}$ & 2.394221000 & -0.630182000 & 0.023420000 \\
$\mathrm{C}$ & -3.023969000 & -2.209463000 & 0.141492000 \\
$\mathrm{C}$ & 3.898460000 & -0.447725000 & -0.032172000 \\
$\mathrm{H}$ & 5.480746000 & -1.142363000 & 0.731581000 \\
$\mathrm{C}$ & -0.557429000 & -2.112741000 & 0.189191000 \\
$\mathrm{~N}$ & 1.790282000 & 0.500481000 & -0.741944000 \\
$\mathrm{O}$ & 4.443001000 & 0.316465000 & -0.791393000 \\
$\mathrm{C}$ & -3.012591000 & -2.178877000 & 1.528449000 \\
$\mathrm{H}$ & 2.492513000 & -2.149386000 & -1.528883000 \\
$\mathrm{C}$ & -0.576373000 & -2.102101000 & 1.591235000 \\
$\mathrm{H}$ & 2.364344000 & 0.698656000 & -1.566824000 \\
$\mathrm{C}$ & -1.800396000 & -2.128631000 & 2.247866000 \\
$\mathrm{H}$ & 0.342649000 & -2.068757000 & 2.161568000 \\
$\mathrm{H}$ & -1.826340000 & -2.145059000 & 3.331231000 \\
$\mathrm{H}$ & 0.825989000 & 0.261773000 & -1.031104000 \\
$\mathrm{H}$ & 1.707958000 & 1.396530000 & -0.184176000 \\
$\mathrm{H}$ & -3.950213000 & -2.213121000 & 2.069551000 \\
$\mathrm{O}$ & 0.510155000 & 0.724540000 & 1.891118000 \\
$\mathrm{H}$ & 1.451200000 & 3.881552000 & 0.292556000 \\
$\mathrm{H}$ & 1.883922000 & 2.869570000 & 1.492986000 \\
$\mathrm{~N}$ & 1.259421000 & 2.968913000 & 0.696867000 \\
$\mathrm{C}$ & -0.398699000 & 1.478259000 & 1.618280000 \\
$\mathrm{H}$ & -1.749044000 & 0.239937000 & 2.066817000 \\
$\mathrm{O}$ & -1.685478000 & 1.172267000 & 1.775835000 \\
$\mathrm{C}$ & -0.141906000 & 2.910632000 & 1.165228000 \\
$\mathrm{H}$ & -0.275950000 & 3.498030000 & 2.085433000 \\
$\mathrm{~S}$ & -1.210562000 & 1.314311000 & -1.703795000 \\
$\mathrm{H}$ & -3.124042000 & 1.146438000 & -0.204278000 \\
$\mathrm{H}$ & -1.643211000 & 3.661559000 & -1.936042000 \\
$\mathrm{H}$ & -3.272611000 & 0.137738000 & -1.656581000 \\
$\mathrm{C}$ & -2.971771000 & 1.112458000 & -1.280344000 \\
$\mathrm{C}$ & -0.955137000 & 3.084173000 & -1.315453000 \\
$\mathrm{C}$ & -1.127220000 & 3.500608000 & 0.145439000 \\
$\mathrm{H}$ & -1.025339000 & 4.590980000 & 0.183279000 \\
$\mathrm{H}$ & -2.139939000 & 3.283415000 & 0.487501000 \\
$\mathrm{H}$ & -3.555194000 & 1.883677000 & -1.784000000 \\
& 0.051441000 & 3.304156000 & -1.676516000
\end{tabular}


TABLE S23: Geometry for WM-A2.

\begin{tabular}{lrrr} 
Element & $\mathrm{X}$ & $\mathrm{Y}$ & $\mathrm{Z}$ \\
\hline $\mathrm{C}$ & 0.111651000 & -2.414105000 & 0.077025000 \\
$\mathrm{C}$ & 1.604088000 & -2.406575000 & 0.168381000 \\
$\mathrm{H}$ & 1.953451000 & -2.795565000 & 1.128049000 \\
$\mathrm{H}$ & -0.316200000 & -3.723043000 & -1.667408000 \\
$\mathrm{H}$ & -2.729252000 & -3.254176000 & -1.168959000 \\
$\mathrm{C}$ & -0.637702000 & -3.073742000 & -0.867660000 \\
$\mathrm{~N}$ & -1.976036000 & -2.822127000 & -0.659005000 \\
$\mathrm{H}$ & 1.933879000 & -0.381665000 & 0.909344000 \\
$\mathrm{C}$ & -2.122158000 & -1.988817000 & 0.432881000 \\
$\mathrm{H}$ & -4.260489000 & -1.691312000 & 0.639763000 \\
$\mathrm{O}$ & 4.316699000 & -1.383041000 & 0.934261000 \\
$\mathrm{C}$ & 2.176732000 & -0.974226000 & 0.032190000 \\
$\mathrm{C}$ & -3.272412000 & -1.458775000 & 1.019526000 \\
$\mathrm{C}$ & 3.680151000 & -0.983343000 & -0.166550000 \\
$\mathrm{H}$ & 5.271252000 & -1.407961000 & 0.757587000 \\
$\mathrm{C}$ & -0.819263000 & -1.707172000 & 0.925828000 \\
$\mathrm{~N}$ & 1.588319000 & -0.272679000 & -1.143826000 \\
$\mathrm{O}$ & 4.210218000 & -0.690440000 & -1.211029000 \\
$\mathrm{C}$ & -3.106534000 & -0.636731000 & 2.125387000 \\
$\mathrm{H}$ & 2.027015000 & -3.049382000 & -0.609334000 \\
$\mathrm{C}$ & -0.684622000 & -0.880800000 & 2.053407000 \\
$\mathrm{H}$ & 2.159554000 & -0.463497000 & -1.973439000 \\
$\mathrm{C}$ & -1.826832000 & -0.354820000 & 2.639413000 \\
$\mathrm{H}$ & 0.289485000 & -0.664010000 & 2.474155000 \\
$\mathrm{H}$ & -1.740004000 & 0.270317000 & 3.519984000 \\
$\mathrm{H}$ & 0.614307000 & -0.571904000 & -1.318635000 \\
$\mathrm{H}$ & 1.536796000 & 0.782779000 & -1.033992000 \\
$\mathrm{H}$ & -3.978765000 & -0.216872000 & 2.611056000 \\
$\mathrm{H}$ & 0.277498000 & 2.778729000 & 3.012287000 \\
$\mathrm{O}$ & 1.496954000 & 1.684961000 & 1.371289000 \\
$\mathrm{H}$ & -3.213905000 & -0.561690000 & -1.739043000 \\
$\mathrm{O}$ & 0.031801000 & 3.187734000 & 2.167909000 \\
$\mathrm{H}$ & -1.398194000 & 1.081623000 & 0.259777000 \\
$\mathrm{C}$ & 0.717366000 & 2.590583000 & 1.186211000 \\
$\mathrm{C}$ & -2.870924000 & 0.061136000 & -2.562544000 \\
$\mathrm{C}$ & -1.686090000 & 1.699742000 & -0.591775000 \\
$\mathrm{H}$ & -2.716831000 & -0.554139000 & -3.448680000 \\
$\mathrm{H}$ & -2.772939000 & 1.777460000 & -0.579741000 \\
$\mathrm{H}$ & -3.603705000 & 0.835469000 & -2.788345000 \\
$\mathrm{~S}$ & -1.261829000 & 0.816812000 & -2.156476000 \\
$\mathrm{C}$ & 0.405054000 & 3.207356000 & -0.169796000 \\
$\mathrm{C}$ & -1.094910000 & 3.107739000 & -0.503717000 \\
$\mathrm{H}$ & -1.627745000 & 3.650938000 & 0.279749000 \\
$\mathrm{~N}$ & 1.258742000 & 2.567284000 & -1.186104000 \\
$\mathrm{H}$ & 0.631911000 & 4.276236000 & -0.069842000 \\
$\mathrm{H}$ & 2.211391000 & 2.914917000 & -1.104532000 \\
$\mathrm{H}$ & -1.275618000 & 3.645105000 & -1.438743000 \\
& 0.932224000 & 2.826894000 & -2.113593000
\end{tabular}


TABLE S24: Geometry for WM-A3.

\begin{tabular}{lrrr} 
Element & $\mathrm{X}$ & $\mathrm{Y}$ & $\mathrm{Z}$ \\
\hline $\mathrm{C}$ & 0.114054000 & -2.414016000 & 0.076471000 \\
$\mathrm{C}$ & 1.606466000 & -2.405313000 & 0.167830000 \\
$\mathrm{H}$ & 1.956140000 & -2.794198000 & 1.127421000 \\
$\mathrm{H}$ & -0.312738000 & -3.722225000 & -1.668782000 \\
$\mathrm{H}$ & -2.726164000 & -3.255199000 & -1.170357000 \\
$\mathrm{C}$ & -0.634766000 & -3.073660000 & -0.868648000 \\
$\mathrm{~N}$ & -1.973287000 & -2.823231000 & -0.659838000 \\
$\mathrm{H}$ & 1.934562000 & -0.380291000 & 0.909304000 \\
$\mathrm{C}$ & -2.120082000 & -1.990668000 & 0.432526000 \\
$\mathrm{H}$ & -4.258645000 & -1.694862000 & 0.639553000 \\
$\mathrm{O}$ & 4.318292000 & -1.379371000 & 0.933939000 \\
$\mathrm{C}$ & 2.177923000 & -0.972398000 & 0.031990000 \\
$\mathrm{C}$ & -3.270745000 & -1.461850000 & 1.019485000 \\
$\mathrm{C}$ & 3.681329000 & -0.980300000 & -0.166844000 \\
$\mathrm{H}$ & 5.272853000 & -1.403509000 & 0.757196000 \\
$\mathrm{C}$ & -0.817422000 & -1.708378000 & 0.925708000 \\
$\mathrm{~N}$ & 1.588854000 & -0.271103000 & -1.143815000 \\
$\mathrm{O}$ & 4.211064000 & -0.687042000 & -1.211401000 \\
$\mathrm{C}$ & -3.105489000 & -0.640368000 & 2.125853000 \\
$\mathrm{H}$ & 2.029925000 & -3.047578000 & -0.610036000 \\
$\mathrm{C}$ & -0.683397000 & -0.882569000 & 2.053776000 \\
$\mathrm{H}$ & 2.160386000 & -0.460981000 & -1.973457000 \\
$\mathrm{C}$ & -1.826001000 & -0.357796000 & 2.640078000 \\
$\mathrm{H}$ & 0.290569000 & -0.665276000 & 2.474593000 \\
$\mathrm{H}$ & -1.739675000 & 0.266897000 & 3.521014000 \\
$\mathrm{H}$ & 0.615160000 & -0.571343000 & -1.318814000 \\
$\mathrm{H}$ & 1.536184000 & 0.784143000 & -1.033658000 \\
$\mathrm{H}$ & -3.978041000 & -0.221457000 & 2.611765000 \\
$\mathrm{O}$ & 1.495053000 & 1.686104000 & 1.371164000 \\
$\mathrm{H}$ & 0.928503000 & 2.828749000 & -2.113235000 \\
$\mathrm{H}$ & 2.207907000 & 2.918115000 & -1.104607000 \\
$\mathrm{~N}$ & 1.255680000 & 2.569268000 & -1.185942000 \\
$\mathrm{C}$ & 0.714646000 & 2.591112000 & 1.186515000 \\
$\mathrm{H}$ & 0.274860000 & 2.778162000 & 3.012715000 \\
$\mathrm{O}$ & 0.028693000 & 3.187279000 & 2.168531000 \\
$\mathrm{C}$ & 0.401511000 & 3.208062000 & -0.169233000 \\
$\mathrm{H}$ & 0.627202000 & 4.277157000 & -0.068888000 \\
$\mathrm{~S}$ & -1.262709000 & 0.816088000 & -2.156133000 \\
$\mathrm{H}$ & -3.214668000 & -0.562899000 & -1.739826000 \\
$\mathrm{H}$ & -1.399130000 & 1.080239000 & 0.260161000 \\
$\mathrm{H}$ & -2.716637000 & -0.555244000 & -3.449168000 \\
$\mathrm{C}$ & -2.871367000 & 0.060018000 & -2.563129000 \\
$\mathrm{C}$ & -1.687913000 & 1.698206000 & -0.591206000 \\
$\mathrm{C}$ & -1.098415000 & 3.106868000 & -0.502881000 \\
$\mathrm{H}$ & -1.279917000 & 3.644296000 & -1.437716000 \\
$\mathrm{H}$ & -1.631735000 & 3.649218000 & 0.280848000 \\
$\mathrm{H}$ & -3.604235000 & 0.834142000 & -2.789345000 \\
& -2.774855000 & 1.774523000 & -0.578995000
\end{tabular}


TABLE S25: Geometry for WM-A4.

\begin{tabular}{lrrr} 
Element & $\mathrm{X}$ & $\mathrm{Y}$ & $\mathrm{Z}$ \\
\hline $\mathrm{C}$ & 1.978686000 & -1.593641000 & 0.769827000 \\
$\mathrm{C}$ & 1.085042000 & -2.778604000 & 0.593184000 \\
$\mathrm{H}$ & 1.548598000 & -3.566106000 & -0.004492000 \\
$\mathrm{H}$ & 2.137607000 & -1.343621000 & 2.972681000 \\
$\mathrm{H}$ & 3.442233000 & 0.730736000 & 2.440061000 \\
$\mathrm{C}$ & 2.334346000 & -1.014322000 & 1.964050000 \\
$\mathrm{~N}$ & 3.041580000 & 0.143210000 & 1.727497000 \\
$\mathrm{H}$ & -0.038681000 & -2.210942000 & -1.176510000 \\
$\mathrm{C}$ & 3.145754000 & 0.347961000 & 0.367384000 \\
$\mathrm{H}$ & 4.211940000 & 2.223729000 & 0.146947000 \\
$\mathrm{O}$ & -0.896785000 & -4.607852000 & -0.496807000 \\
$\mathrm{C}$ & -0.235568000 & -2.376484000 & -0.115597000 \\
$\mathrm{C}$ & 3.722591000 & 1.396000000 & -0.352759000 \\
$\mathrm{C}$ & -1.316941000 & -3.440438000 & -0.003377000 \\
$\mathrm{H}$ & -1.608834000 & -5.262444000 & -0.411712000 \\
$\mathrm{C}$ & 2.500611000 & -0.745761000 & -0.272603000 \\
$\mathrm{~N}$ & -0.779016000 & -1.105030000 & 0.432791000 \\
$\mathrm{O}$ & -2.406456000 & -3.237057000 & 0.469740000 \\
$\mathrm{C}$ & 3.659558000 & 1.333751000 & -1.738658000 \\
$\mathrm{H}$ & 0.850514000 & -3.216260000 & 1.567861000 \\
$\mathrm{C}$ & 2.477002000 & -0.794668000 & -1.676302000 \\
$\mathrm{H}$ & -1.805986000 & -1.135804000 & 0.494190000 \\
$\mathrm{C}$ & 3.050951000 & 0.245364000 & -2.395269000 \\
$\mathrm{H}$ & 2.035136000 & -1.635657000 & -2.198956000 \\
$\mathrm{H}$ & 3.047509000 & 0.215764000 & -3.477983000 \\
$\mathrm{H}$ & -0.390967000 & -0.872564000 & 1.349739000 \\
$\mathrm{H}$ & -0.545741000 & -0.256151000 & -0.177539000 \\
$\mathrm{H}$ & 4.103964000 & 2.126874000 & -2.327117000 \\
$\mathrm{H}$ & 0.286901000 & 3.198641000 & -0.902368000 \\
$\mathrm{O}$ & 0.207051000 & 3.546626000 & 1.519901000 \\
$\mathrm{C}$ & -0.383830000 & 2.443440000 & -0.480619000 \\
$\mathrm{H}$ & -1.821181000 & 4.008714000 & -0.261738000 \\
$\mathrm{C}$ & -0.049330000 & 2.337096000 & 1.000943000 \\
$\mathrm{C}$ & -1.822047000 & 2.984648000 & -0.642626000 \\
$\mathrm{H}$ & -2.037227000 & 3.050915000 & -1.713346000 \\
$\mathrm{H}$ & 0.787069000 & 0.999733000 & -1.365294000 \\
$\mathrm{~N}$ & -0.195588000 & 1.139595000 & -1.135763000 \\
$\mathrm{O}$ & -0.050063000 & 1.322299000 & 1.658881000 \\
$\mathrm{C}$ & -2.947538000 & 2.226933000 & 0.065898000 \\
$\mathrm{H}$ & -0.698204000 & 1.132319000 & -2.019406000 \\
$\mathrm{H}$ & -5.696158000 & 1.135158000 & -0.436699000 \\
$\mathrm{H}$ & -3.855832000 & 2.827507000 & -0.004980000 \\
$\mathrm{H}$ & -2.726444000 & 2.083209000 & 1.126247000 \\
$\mathrm{C}$ & -5.033320000 & 0.355012000 & -0.062399000 \\
$\mathrm{~S}$ & -3.341036000 & 0.599486000 & -0.700056000 \\
$\mathrm{H}$ & -5.039458000 & 0.340766000 & 1.027452000 \\
$\mathrm{H}$ & -5.371150000 & -0.612219000 & -0.433155000 \\
& 0.346352000 & 3.451655000 & 2.475156000
\end{tabular}


TABLE S26: Geometry for WM-A5.

\begin{tabular}{lrrr} 
Element & $\mathrm{X}$ & $\mathrm{Y}$ & $\mathrm{Z}$ \\
\hline $\mathrm{H}$ & -4.026902000 & 1.838454000 & 1.847810000 \\
$\mathrm{H}$ & 0.178842000 & -1.339050000 & 1.271410000 \\
$\mathrm{C}$ & -4.184751000 & 0.858514000 & 1.398200000 \\
$\mathrm{~N}$ & 0.093402000 & -1.450380000 & 0.264540000 \\
$\mathrm{H}$ & -4.899960000 & 0.301303000 & 2.003530000 \\
$\mathrm{~S}$ & -2.567110000 & 0.013156000 & 1.389990000 \\
$\mathrm{H}$ & -4.562901000 & 0.989073000 & 0.384310000 \\
$\mathrm{C}$ & -0.753886000 & -2.607841000 & -0.056000000 \\
$\mathrm{H}$ & -1.678096000 & -2.735643000 & 1.882170000 \\
$\mathrm{O}$ & -1.013858000 & -1.566262000 & -2.237150000 \\
$\mathrm{H}$ & -0.211685000 & -3.551320000 & 0.073980000 \\
$\mathrm{C}$ & -2.007246000 & -2.695123000 & 0.839540000 \\
$\mathrm{C}$ & -3.067127000 & -1.604295000 & 0.670700000 \\
$\mathrm{C}$ & -1.154206000 & -2.533732000 & -1.524390000 \\
$\mathrm{H}$ & -3.335538000 & -1.468135000 & -0.380540000 \\
$\mathrm{H}$ & -3.967737000 & -1.915666000 & 1.202510000 \\
$\mathrm{H}$ & -2.483084000 & -3.654704000 & 0.626350000 \\
$\mathrm{O}$ & -1.706734000 & -3.681443000 & -1.936040000 \\
$\mathrm{H}$ & -1.962775000 & -3.589693000 & -2.867560000 \\
$\mathrm{H}$ & 1.043723000 & -1.599609000 & -0.068370000 \\
$\mathrm{H}$ & 2.528851000 & -0.445996000 & 3.628640000 \\
$\mathrm{C}$ & 2.753781000 & -0.442616000 & 2.569070000 \\
$\mathrm{H}$ & 1.656598000 & 1.363302000 & 2.198870000 \\
$\mathrm{H}$ & 3.945743000 & -2.236174000 & 2.691880000 \\
$\mathrm{C}$ & 2.258949000 & 0.573333000 & 1.764750000 \\
$\mathrm{C}$ & 3.566802000 & -1.462385000 & 2.035770000 \\
$\mathrm{H}$ & -0.143832000 & 1.822510000 & 0.781050000 \\
$\mathrm{O}$ & -0.880986000 & 4.308159000 & 0.204970000 \\
$\mathrm{H}$ & -1.585327000 & 4.964828000 & 0.080710000 \\
$\mathrm{C}$ & -1.213924000 & 3.186188000 & -0.438020000 \\
$\mathrm{C}$ & -0.147793000 & 2.115530000 & -0.269750000 \\
$\mathrm{C}$ & 2.572969000 & 0.570664000 & 0.395280000 \\
$\mathrm{C}$ & 3.907722000 & -1.483924000 & 0.690350000 \\
$\mathrm{H}$ & 1.583875000 & 3.343862000 & 0.014850000 \\
$\mathrm{C}$ & 1.268106000 & 2.571402000 & -0.689540000 \\
$\mathrm{C}$ & 3.404821000 & -0.461415000 & -0.118130000 \\
$\mathrm{C}$ & 2.217658000 & 1.416633000 & -0.717200000 \\
$\mathrm{H}$ & 4.547184000 & -2.260593000 & 0.287780000 \\
$\mathrm{H}$ & 1.219986000 & 3.043212000 & -1.675780000 \\
$\mathrm{~N}$ & 3.546211000 & -0.235975000 & -1.474280000 \\
$\mathrm{C}$ & 2.835679000 & 0.892494000 & -1.826700000 \\
$\mathrm{H}$ & 4.148391000 & -0.755354000 & -2.092460000 \\
$\mathrm{H}$ & 2.834438000 & 1.245874000 & -2.846540000 \\
$\mathrm{H}$ & -0.317640000 & 0.002119000 & -0.532830000 \\
$\mathrm{~N}$ & -0.577311000 & 0.916309000 & -1.037760000 \\
& -1.595451000 & 0.927498000 & -1.143690000 \\
& -2.231504000 & 3.021257000 & -1.063130000 \\
-0.159731000 & 0.875470000 & -1.968150000
\end{tabular}


TABLE S27: Geometry for WM-A6.

\begin{tabular}{lrrr} 
Element & $\mathrm{X}$ & $\mathrm{Y}$ & $\mathrm{Z}$ \\
\hline $\mathrm{C}$ & 1.978686000 & -1.593641000 & 0.769827000 \\
$\mathrm{C}$ & 1.085042000 & -2.778604000 & 0.593184000 \\
$\mathrm{H}$ & 1.548598000 & -3.566106000 & -0.004492000 \\
$\mathrm{H}$ & 2.137607000 & -1.343621000 & 2.972681000 \\
$\mathrm{H}$ & 3.442233000 & 0.730736000 & 2.440061000 \\
$\mathrm{C}$ & 2.334346000 & -1.014322000 & 1.964050000 \\
$\mathrm{~N}$ & 3.041580000 & 0.143210000 & 1.727497000 \\
$\mathrm{H}$ & -0.038681000 & -2.210942000 & -1.176510000 \\
$\mathrm{C}$ & 3.145754000 & 0.347961000 & 0.367384000 \\
$\mathrm{H}$ & 4.211940000 & 2.223729000 & 0.146947000 \\
$\mathrm{O}$ & -0.896785000 & -4.607852000 & -0.496807000 \\
$\mathrm{C}$ & -0.235568000 & -2.376484000 & -0.115597000 \\
$\mathrm{C}$ & 3.722591000 & 1.396000000 & -0.352759000 \\
$\mathrm{C}$ & -1.316941000 & -3.440438000 & -0.003377000 \\
$\mathrm{H}$ & -1.608834000 & -5.262444000 & -0.411712000 \\
$\mathrm{C}$ & 2.500611000 & -0.745761000 & -0.272603000 \\
$\mathrm{~N}$ & -0.779016000 & -1.105030000 & 0.432791000 \\
$\mathrm{O}$ & -2.406456000 & -3.237057000 & 0.469740000 \\
$\mathrm{C}$ & 3.659558000 & 1.333751000 & -1.738658000 \\
$\mathrm{H}$ & 0.850514000 & -3.216260000 & 1.567861000 \\
$\mathrm{C}$ & 2.477002000 & -0.794668000 & -1.676302000 \\
$\mathrm{H}$ & -1.805986000 & -1.135804000 & 0.494190000 \\
$\mathrm{C}$ & 3.050951000 & 0.245364000 & -2.395269000 \\
$\mathrm{H}$ & 2.035136000 & -1.635657000 & -2.198956000 \\
$\mathrm{H}$ & 3.047509000 & 0.215764000 & -3.477983000 \\
$\mathrm{H}$ & -0.390967000 & -0.872564000 & 1.349739000 \\
$\mathrm{H}$ & -0.545741000 & -0.256151000 & -0.177539000 \\
$\mathrm{H}$ & 4.103964000 & 2.126874000 & -2.327117000 \\
$\mathrm{H}$ & 0.286901000 & 3.198641000 & -0.902368000 \\
$\mathrm{O}$ & 0.207051000 & 3.546626000 & 1.519901000 \\
$\mathrm{C}$ & -0.383830000 & 2.443440000 & -0.480619000 \\
$\mathrm{H}$ & -1.821181000 & 4.008714000 & -0.261738000 \\
$\mathrm{C}$ & -0.049330000 & 2.337096000 & 1.000943000 \\
$\mathrm{C}$ & -1.822047000 & 2.984648000 & -0.642626000 \\
$\mathrm{H}$ & -2.037227000 & 3.050915000 & -1.713346000 \\
$\mathrm{H}$ & 0.787069000 & 0.999733000 & -1.365294000 \\
$\mathrm{~N}$ & -0.195588000 & 1.139595000 & -1.135763000 \\
$\mathrm{O}$ & -0.050063000 & 1.322299000 & 1.658881000 \\
$\mathrm{C}$ & -2.947538000 & 2.226933000 & 0.065898000 \\
$\mathrm{H}$ & -0.698204000 & 1.132319000 & -2.019406000 \\
$\mathrm{H}$ & -5.696158000 & 1.135158000 & -0.436699000 \\
$\mathrm{H}$ & -3.855832000 & 2.827507000 & -0.004980000 \\
$\mathrm{H}$ & -2.726444000 & 2.083209000 & 1.126247000 \\
$\mathrm{C}$ & -5.033320000 & 0.355012000 & -0.062399000 \\
$\mathrm{~S}$ & -3.341036000 & 0.599486000 & -0.700056000 \\
$\mathrm{H}$ & -5.039458000 & 0.340766000 & 1.027452000 \\
$\mathrm{H}$ & -5.371150000 & -0.612219000 & -0.433155000 \\
& 0.346352000 & 3.451655000 & 2.475156000
\end{tabular}


TABLE S28: Geometry for WM-A7.

\begin{tabular}{lrrr} 
Element & $\mathrm{X}$ & $\mathrm{Y}$ & $\mathrm{Z}$ \\
\hline $\mathrm{C}$ & 2.131100000 & 1.419263000 & -0.782263000 \\
$\mathrm{C}$ & 1.150073000 & 2.542143000 & -0.678179000 \\
$\mathrm{H}$ & 1.496068000 & 3.322328000 & 0.003908000 \\
$\mathrm{H}$ & 2.615948000 & 1.301867000 & -2.948031000 \\
$\mathrm{H}$ & 4.078734000 & -0.634040000 & -2.304428000 \\
$\mathrm{C}$ & 2.699539000 & 0.937685000 & -1.935617000 \\
$\mathrm{~N}$ & 3.476432000 & -0.165698000 & -1.646492000 \\
$\mathrm{H}$ & -0.119411000 & 1.736697000 & 0.882703000 \\
$\mathrm{C}$ & 3.434466000 & -0.410994000 & -0.286371000 \\
$\mathrm{H}$ & 4.668441000 & -2.168735000 & 0.015390000 \\
$\mathrm{O}$ & -0.957271000 & 4.180149000 & 0.559070000 \\
$\mathrm{C}$ & -0.222540000 & 2.057679000 & -0.155343000 \\
$\mathrm{C}$ & 4.021279000 & -1.427578000 & 0.469367000 \\
$\mathrm{C}$ & -1.285132000 & 3.142140000 & -0.220026000 \\
$\mathrm{H}$ & -1.641462000 & 4.864311000 & 0.477480000 \\
$\mathrm{C}$ & 2.590313000 & 0.574734000 & 0.292729000 \\
$\mathrm{~N}$ & -0.707369000 & 0.879766000 & -0.915369000 \\
$\mathrm{O}$ & -2.282116000 & 3.064196000 & -0.889564000 \\
$\mathrm{C}$ & 3.747570000 & -1.453445000 & 1.830166000 \\
$\mathrm{H}$ & 1.013532000 & 3.014596000 & -1.655865000 \\
$\mathrm{C}$ & 2.346464000 & 0.532192000 & 1.675381000 \\
$\mathrm{H}$ & -0.696750000 & 1.024539000 & -1.925309000 \\
$\mathrm{C}$ & 2.920044000 & -0.482418000 & 2.428326000 \\
$\mathrm{H}$ & 1.740082000 & 1.288906000 & 2.160532000 \\
$\mathrm{H}$ & 2.748980000 & -0.520541000 & 3.497226000 \\
$\mathrm{H}$ & -0.150024000 & -0.003694000 & -0.694937000 \\
$\mathrm{H}$ & -1.683971000 & 0.638212000 & -0.646296000 \\
$\mathrm{H}$ & 4.190739000 & -2.225545000 & 2.446822000 \\
$\mathrm{O}$ & -1.393708000 & -1.463329000 & -2.278640000 \\
$\mathrm{H}$ & 0.952925000 & -1.677579000 & 0.593725000 \\
$\mathrm{H}$ & 1.054118000 & -1.789650000 & -1.025385000 \\
$\mathrm{~N}$ & 0.406470000 & -1.615212000 & -0.261814000 \\
$\mathrm{C}$ & -1.573484000 & -2.342502000 & -1.467867000 \\
$\mathrm{H}$ & -3.103462000 & -3.039912000 & -2.328746000 \\
$\mathrm{O}$ & -2.566910000 & -3.234389000 & -1.544024000 \\
$\mathrm{C}$ & -0.674692000 & -2.609502000 & -0.263656000 \\
$\mathrm{H}$ & -0.295639000 & -3.628245000 & -0.418719000 \\
$\mathrm{~S}$ & -3.168092000 & -0.462342000 & 0.673518000 \\
$\mathrm{H}$ & -4.036705000 & 0.527245000 & 2.713449000 \\
$\mathrm{H}$ & -2.279000000 & -1.469919000 & 2.662793000 \\
$\mathrm{H}$ & -4.283094000 & 1.552166000 & 1.280680000 \\
$\mathrm{C}$ & -3.574596000 & 0.912109000 & 1.804741000 \\
$\mathrm{C}$ & -1.863489000 & -1.294507000 & 1.669133000 \\
$\mathrm{C}$ & -1.425530000 & -2.638469000 & 1.084188000 \\
$\mathrm{H}$ & -0.755596000 & -3.102140000 & 1.815144000 \\
$\mathrm{H}$ & -2.284783000 & -3.303098000 & 0.984707000 \\
$\mathrm{H}$ & -2.685453000 & 1.491765000 & 2.058398000 \\
& -1.011741000 & -0.618257000 & 1.781199000
\end{tabular}


TABLE S29: Geometry for WM-A8.

\begin{tabular}{lrrr} 
Element & $\mathrm{X}$ & $\mathrm{Y}$ & $\mathrm{Z}$ \\
\hline $\mathrm{O}$ & 1.226346000 & 4.614259000 & 0.583012000 \\
$\mathrm{O}$ & 2.151272000 & 2.891021000 & 1.711215000 \\
$\mathrm{~N}$ & -3.269523000 & 1.003412000 & -1.959983000 \\
$\mathrm{~N}$ & 0.806391000 & 1.036268000 & 0.340154000 \\
$\mathrm{C}$ & -2.002058000 & 1.844741000 & -0.300956000 \\
$\mathrm{C}$ & -1.052722000 & 2.717525000 & 0.458756000 \\
$\mathrm{C}$ & -2.525613000 & 0.570426000 & 0.124141000 \\
$\mathrm{C}$ & 0.425522000 & 2.448253000 & 0.091973000 \\
$\mathrm{C}$ & -3.310559000 & 0.064653000 & -0.948634000 \\
$\mathrm{C}$ & -2.491659000 & 2.070860000 & -1.565044000 \\
$\mathrm{C}$ & -2.394040000 & -0.198098000 & 1.291732000 \\
$\mathrm{C}$ & -3.931108000 & -1.186021000 & -0.892101000 \\
$\mathrm{C}$ & 1.379018000 & 3.322680000 & 0.894486000 \\
$\mathrm{C}$ & -3.009578000 & -1.438877000 & 1.351248000 \\
$\mathrm{C}$ & -3.765542000 & -1.930665000 & 0.268149000 \\
$\mathrm{H}$ & -1.242314000 & 3.773007000 & 0.257821000 \\
$\mathrm{H}$ & -1.182670000 & 2.575536000 & 1.536374000 \\
$\mathrm{H}$ & 0.570872000 & 2.644113000 & -0.972052000 \\
$\mathrm{H}$ & -2.353564000 & 2.920010000 & -2.216595000 \\
$\mathrm{H}$ & -3.791280000 & 0.957039000 & -2.820328000 \\
$\mathrm{H}$ & -1.815944000 & 0.161032000 & 2.134644000 \\
$\mathrm{H}$ & -4.525287000 & -1.560578000 & -1.717123000 \\
$\mathrm{H}$ & -2.921417000 & -2.039395000 & 2.248442000 \\
$\mathrm{H}$ & 1.765903000 & 0.822704000 & 0.004968000 \\
$\mathrm{H}$ & 0.234489000 & 0.309595000 & -0.166525000 \\
$\mathrm{H}$ & -4.239103000 & -2.901339000 & 0.348216000 \\
$\mathrm{H}$ & 1.829525000 & 5.143981000 & 1.129227000 \\
$\mathrm{H}$ & 0.780080000 & 0.779276000 & 1.329444000 \\
$\mathrm{H}$ & 3.885040000 & -2.640332000 & -0.667046000 \\
$\mathrm{C}$ & 4.813111000 & -0.087824000 & -1.074641000 \\
$\mathrm{H}$ & 2.998590000 & -1.875920000 & 0.652613000 \\
$\mathrm{~S}$ & 3.059948000 & -0.513586000 & -1.358470000 \\
$\mathrm{C}$ & 2.973658000 & -2.095190000 & -0.416605000 \\
$\mathrm{O}$ & 0.627185000 & -1.307189000 & 1.802857000 \\
$\mathrm{H}$ & 5.457681000 & -0.855180000 & -1.502968000 \\
$\mathrm{C}$ & 1.777148000 & -2.976518000 & -0.785109000 \\
$\mathrm{C}$ & 0.438610000 & -2.355520000 & 1.228489000 \\
$\mathrm{H}$ & 1.971121000 & -3.971805000 & -0.377289000 \\
$\mathrm{H}$ & & &
\end{tabular}


TABLE S30: Geometry for WM-B1.

\begin{tabular}{lrrr} 
Element & $\mathrm{X}$ & $\mathrm{Y}$ & $\mathrm{Z}$ \\
\hline $\mathrm{C}$ & 2.085975000 & -1.374750000 & -0.410943000 \\
$\mathrm{C}$ & 3.049443000 & -0.267661000 & -0.129205000 \\
$\mathrm{H}$ & 3.660018000 & -0.486034000 & 0.750629000 \\
$\mathrm{H}$ & 2.500861000 & -1.932415000 & -2.523390000 \\
$\mathrm{H}$ & 0.757345000 & -3.700680000 & -2.184543000 \\
$\mathrm{C}$ & 1.949703000 & -2.047521000 & -1.602522000 \\
$\mathrm{~N}$ & 0.973165000 & -3.011577000 & -1.482532000 \\
$\mathrm{H}$ & 1.763039000 & 1.034014000 & 1.064219000 \\
$\mathrm{C}$ & 0.464680000 & -2.992486000 & -0.197424000 \\
$\mathrm{H}$ & -0.996139000 & -4.594455000 & -0.137587000 \\
$\mathrm{O}$ & 4.012865000 & 2.227082000 & 1.343194000 \\
$\mathrm{C}$ & 2.338414000 & 1.079555000 & 0.140977000 \\
$\mathrm{C}$ & -0.505138000 & -3.794972000 & 0.404834000 \\
$\mathrm{C}$ & 3.338483000 & 2.221802000 & 0.193736000 \\
$\mathrm{H}$ & 4.672356000 & 2.940019000 & 1.322637000 \\
$\mathrm{C}$ & 1.136631000 & -1.957380000 & 0.506556000 \\
$\mathrm{~N}$ & 1.376092000 & 1.400521000 & -0.953023000 \\
$\mathrm{O}$ & 3.509181000 & 2.982001000 & -0.725925000 \\
$\mathrm{C}$ & -0.804259000 & -3.545144000 & 1.737852000 \\
$\mathrm{H}$ & 3.737418000 & -0.145864000 & -0.971285000 \\
$\mathrm{C}$ & 0.809379000 & -1.726363000 & 1.851548000 \\
$\mathrm{H}$ & 1.872379000 & 1.831000000 & -1.739233000 \\
$\mathrm{C}$ & -0.156041000 & -2.520512000 & 2.453193000 \\
$\mathrm{H}$ & 1.317197000 & -0.960226000 & 2.426772000 \\
$\mathrm{H}$ & -0.406554000 & -2.363561000 & 3.495186000 \\
$\mathrm{H}$ & 0.868971000 & 0.549437000 & -1.245415000 \\
$\mathrm{H}$ & 0.652375000 & 2.062829000 & -0.587247000 \\
$\mathrm{H}$ & -1.542894000 & -4.157891000 & 2.239916000 \\
$\mathrm{H}$ & -3.175681000 & 3.395428000 & -0.570682000 \\
$\mathrm{H}$ & -4.272651000 & 1.310393000 & -0.332338000 \\
$\mathrm{O}$ & -2.191449000 & 3.512630000 & -0.577346000 \\
$\mathrm{H}$ & -3.216214000 & -1.407715000 & -2.596742000 \\
$\mathrm{~N}$ & -4.089791000 & 2.035759000 & 0.352699000 \\
$\mathrm{H}$ & -4.877480000 & 2.093080000 & 0.985915000 \\
$\mathrm{H}$ & -1.665336000 & -1.210585000 & -3.436801000 \\
$\mathrm{C}$ & -2.133412000 & -1.397337000 & -2.470887000 \\
$\mathrm{~S}$ & -1.634261000 & -0.044446000 & -1.357803000 \\
$\mathrm{C}$ & -1.738105000 & 2.634414000 & 0.286645000 \\
$\mathrm{H}$ & -3.540671000 & -0.756178000 & -0.091445000 \\
$\mathrm{C}$ & -2.813523000 & 1.842071000 & 1.044412000 \\
$\mathrm{H}$ & -1.789576000 & -2.352399000 & -2.075860000 \\
$\mathrm{C}$ & -2.492145000 & -0.594269000 & 0.173276000 \\
$\mathrm{H}$ & -2.882493000 & 2.355581000 & 2.009722000 \\
$\mathrm{O}$ & -0.544062000 & 2.482654000 & 0.499897000 \\
$\mathrm{C}$ & -2.366268000 & 0.394971000 & 1.329607000 \\
$\mathrm{H}$ & -2.073891000 & -1.552419000 & 0.477505000 \\
$\mathrm{H}$ & -2.970403000 & 0.003425000 & 2.152606000 \\
& -1.335255000 & 0.421991000 & 1.683813000
\end{tabular}


TABLE S31: Geometry for WM-B2.

\begin{tabular}{lrrr} 
Element & $\mathrm{X}$ & $\mathrm{Y}$ & $\mathrm{Z}$ \\
\hline $\mathrm{C}$ & 2.640961000 & 1.116333000 & 0.514426000 \\
$\mathrm{C}$ & 3.550335000 & 0.017500000 & 0.065244000 \\
$\mathrm{H}$ & 3.983796000 & 0.238702000 & -0.912265000 \\
$\mathrm{H}$ & 3.229928000 & 1.515030000 & 2.623011000 \\
$\mathrm{H}$ & 1.428818000 & 3.254792000 & 2.586669000 \\
$\mathrm{C}$ & 2.604143000 & 1.692961000 & 1.761385000 \\
$\mathrm{~N}$ & 1.618076000 & 2.653876000 & 1.801024000 \\
$\mathrm{H}$ & 2.013466000 & -1.261674000 & -0.789105000 \\
$\mathrm{C}$ & 1.005070000 & 2.733426000 & 0.567195000 \\
$\mathrm{H}$ & -0.463144000 & 4.318022000 & 0.757604000 \\
$\mathrm{O}$ & 4.382752000 & -2.229697000 & -1.589494000 \\
$\mathrm{C}$ & 2.821048000 & -1.336017000 & -0.058179000 \\
$\mathrm{C}$ & -0.011032000 & 3.575303000 & 0.111054000 \\
$\mathrm{C}$ & 3.724188000 & -2.492941000 & -0.462465000 \\
$\mathrm{H}$ & 4.930760000 & -2.996286000 & -1.825403000 \\
$\mathrm{C}$ & 1.623034000 & 1.767524000 & -0.272936000 \\
$\mathrm{~N}$ & 2.179627000 & -1.717809000 & 1.242968000 \\
$\mathrm{O}$ & 3.802767000 & -3.516499000 & 0.171167000 \\
$\mathrm{C}$ & -0.404448000 & 3.443861000 & -1.214984000 \\
$\mathrm{H}$ & 4.389825000 & -0.092546000 & 0.760369000 \\
$\mathrm{C}$ & 1.199399000 & 1.654581000 & -1.606173000 \\
$\mathrm{H}$ & 2.194472000 & -2.741078000 & 1.328303000 \\
$\mathrm{C}$ & 0.194610000 & 2.493749000 & -2.065247000 \\
$\mathrm{H}$ & 1.661003000 & 0.940684000 & -2.278872000 \\
$\mathrm{H}$ & -0.121637000 & 2.433757000 & -3.099725000 \\
$\mathrm{H}$ & 2.705635000 & -1.337905000 & 2.031697000 \\
$\mathrm{H}$ & 1.178694000 & -1.339078000 & 1.303596000 \\
$\mathrm{H}$ & -1.173959000 & 4.098278000 & -1.606691000 \\
$\mathrm{H}$ & -1.555032000 & 1.392482000 & 0.031794000 \\
$\mathrm{O}$ & -0.245059000 & -0.649069000 & 1.308192000 \\
$\mathrm{C}$ & -1.972075000 & 0.385744000 & -0.059003000 \\
$\mathrm{H}$ & -2.625634000 & 0.525559000 & 1.967696000 \\
$\mathrm{C}$ & -0.786504000 & -0.538784000 & 0.214474000 \\
$\mathrm{C}$ & -3.067282000 & 0.234273000 & 1.010404000 \\
$\mathrm{H}$ & -3.854072000 & 0.961305000 & 0.794606000 \\
$\mathrm{H}$ & -2.415094000 & 1.034670000 & -1.979806000 \\
$\mathrm{~N}$ & -2.368138000 & 0.168135000 & -1.458662000 \\
$\mathrm{O}$ & -0.365913000 & -1.199416000 & -0.844272000 \\
$\mathrm{H}$ & -1.011106000 & -0.880997000 & -1.562263000 \\
$\mathrm{C}$ & -3.660949000 & -1.167326000 & 1.173565000 \\
$\mathrm{H}$ & -3.251875000 & -0.336164000 & -1.526055000 \\
$\mathrm{H}$ & -5.977301000 & 0.287949000 & -0.136596000 \\
$\mathrm{H}$ & -4.277961000 & -1.203703000 & 2.073698000 \\
$\mathrm{H}$ & -2.871830000 & -1.911548000 & 1.305455000 \\
$\mathrm{C}$ & -6.181746000 & -0.774909000 & -0.005095000 \\
$\mathrm{~S}$ & -4.680396000 & -1.790854000 & -0.222375000 \\
$\mathrm{H}$ & -6.619588000 & -0.955303000 & 0.977268000 \\
$\mathrm{H}$ & -6.887675000 & -1.094919000 & -0.771040000
\end{tabular}


TABLE S32: Geometry for WM-Z1.

\begin{tabular}{lrrr} 
Element & $\mathrm{X}$ & $\mathrm{Y}$ & $\mathrm{Z}$ \\
\hline $\mathrm{C}$ & -2.306249000 & 1.382692000 & -0.627105000 \\
$\mathrm{C}$ & -3.511899000 & 0.641316000 & -0.141400000 \\
$\mathrm{H}$ & -3.880715000 & 1.074283000 & 0.790269000 \\
$\mathrm{H}$ & -2.616721000 & 1.611332000 & -2.815729000 \\
$\mathrm{H}$ & -0.398370000 & 2.769236000 & -2.813507000 \\
$\mathrm{C}$ & -2.028465000 & 1.744358000 & -1.920800000 \\
$\mathrm{~N}$ & -0.802434000 & 2.372645000 & -1.980904000 \\
$\mathrm{H}$ & -2.393260000 & -0.956360000 & 0.822307000 \\
$\mathrm{C}$ & -0.266484000 & 2.449422000 & -0.714225000 \\
$\mathrm{H}$ & 1.612687000 & 3.507578000 & -0.946420000 \\
$\mathrm{O}$ & -4.895093000 & -0.999266000 & 1.805730000 \\
$\mathrm{C}$ & -3.232435000 & -0.851052000 & 0.128454000 \\
$\mathrm{C}$ & 0.926397000 & 3.019108000 & -0.264442000 \\
$\mathrm{C}$ & -4.423131000 & -1.598367000 & 0.707570000 \\
$\mathrm{H}$ & -5.632876000 & -1.524807000 & 2.155051000 \\
$\mathrm{C}$ & -1.192140000 & 1.828600000 & 0.171682000 \\
$\mathrm{~N}$ & -2.813428000 & -1.555763000 & -1.119282000 \\
$\mathrm{O}$ & -4.862392000 & -2.617589000 & 0.236226000 \\
$\mathrm{C}$ & 1.182530000 & 2.978747000 & 1.103083000 \\
$\mathrm{H}$ & -4.329000000 & 0.727562000 & -0.866160000 \\
$\mathrm{C}$ & -0.902994000 & 1.794630000 & 1.543325000 \\
$\mathrm{H}$ & -3.009943000 & -2.554880000 & -1.016003000 \\
$\mathrm{C}$ & 0.273529000 & 2.375582000 & 1.999344000 \\
$\mathrm{H}$ & -1.590590000 & 1.336034000 & 2.243946000 \\
$\mathrm{H}$ & 0.490249000 & 2.386959000 & 3.061053000 \\
$\mathrm{H}$ & -3.358462000 & -1.235152000 & -1.919835000 \\
$\mathrm{H}$ & -1.714691000 & -1.402488000 & -1.325393000 \\
$\mathrm{H}$ & 2.078359000 & 3.450261000 & 1.490207000 \\
$\mathrm{O}$ & -0.317793000 & -1.154036000 & -1.512541000 \\
$\mathrm{H}$ & 2.280078000 & -1.947142000 & -1.928914000 \\
$\mathrm{C}$ & 0.248008000 & -1.112288000 & -0.385498000 \\
$\mathrm{H}$ & 1.782207000 & 0.289851000 & -0.998977000 \\
$\mathrm{C}$ & 2.650182000 & -1.698488000 & -0.931803000 \\
$\mathrm{C}$ & 1.709724000 & -0.614699000 & -0.396730000 \\
$\mathrm{O}$ & -0.225809000 & -1.432781000 & 0.719757000 \\
$\mathrm{H}$ & 4.214489000 & -0.279054000 & -1.446227000 \\
$\mathrm{H}$ & 4.654733000 & -1.973353000 & -1.698942000 \\
$\mathrm{C}$ & 4.122053000 & -1.289080000 & -1.038070000 \\
$\mathrm{H}$ & 6.928797000 & -0.192934000 & 1.250086000 \\
$\mathrm{H}$ & 2.549061000 & -2.608933000 & -0.331103000 \\
$\mathrm{~N}$ & 2.017072000 & -0.240714000 & 1.029799000 \\
$\mathrm{H}$ & 1.762014000 & 0.738961000 & 1.213333000 \\
$\mathrm{H}$ & 7.011500000 & -0.679311000 & -0.453884000 \\
$\mathrm{H}$ & 6.054301000 & 0.755517000 & 0.024209000 \\
& 1.373978000 & -0.828368000 & 1.593371000 \\
$\mathrm{H}$ & 4.936790000 & -1.330990000 & 0.616666000 \\
& 3.017579000 & -0.400759000 & 1.260044000 \\
& 6.372403000 & -0.245811000 & 0.314934000 \\
& & &
\end{tabular}


TABLE S33: Geometry for WM-Z2.

\begin{tabular}{lrrr} 
Element & $\mathrm{X}$ & $\mathrm{Y}$ & $\mathrm{Z}$ \\
\hline $\mathrm{C}$ & 2.406375000 & 1.192053000 & 0.559853000 \\
$\mathrm{C}$ & 3.430021000 & 0.200798000 & 0.100719000 \\
$\mathrm{H}$ & 3.857414000 & 0.510699000 & -0.854689000 \\
$\mathrm{H}$ & 2.792118000 & 1.450679000 & 2.733842000 \\
$\mathrm{H}$ & 0.893978000 & 3.079788000 & 2.679002000 \\
$\mathrm{C}$ & 2.232935000 & 1.665213000 & 1.836067000 \\
$\mathrm{~N}$ & 1.175287000 & 2.550035000 & 1.870095000 \\
$\mathrm{H}$ & 1.994488000 & -1.188475000 & -0.757568000 \\
$\mathrm{C}$ & 0.656483000 & 2.689448000 & 0.599912000 \\
$\mathrm{H}$ & -0.938048000 & 4.149389000 & 0.785513000 \\
$\mathrm{O}$ & 4.412472000 & -1.740920000 & -1.808776000 \\
$\mathrm{C}$ & 2.864559000 & -1.220065000 & -0.095215000 \\
$\mathrm{C}$ & -0.387543000 & 3.487997000 & 0.126409000 \\
$\mathrm{C}$ & 3.868849000 & -2.201953000 & -0.678760000 \\
$\mathrm{H}$ & 5.020607000 & -2.411542000 & -2.159637000 \\
$\mathrm{C}$ & 1.408035000 & 1.837721000 & -0.256574000 \\
$\mathrm{~N}$ & 2.371864000 & -1.793545000 & 1.194428000 \\
$\mathrm{O}$ & 4.114207000 & -3.273688000 & -0.182771000 \\
$\mathrm{C}$ & -0.666726000 & 3.438760000 & -1.236486000 \\
$\mathrm{H}$ & 4.263539000 & 0.158699000 & 0.810732000 \\
$\mathrm{C}$ & 1.098188000 & 1.803790000 & -1.624714000 \\
$\mathrm{H}$ & 2.434442000 & -2.814790000 & 1.142498000 \\
$\mathrm{C}$ & 0.073695000 & 2.607417000 & -2.104242000 \\
$\mathrm{H}$ & 1.656805000 & 1.170558000 & -2.303707000 \\
$\mathrm{H}$ & -0.153972000 & 2.612034000 & -3.163539000 \\
$\mathrm{H}$ & 2.966327000 & -1.505593000 & 1.971991000 \\
$\mathrm{H}$ & 1.316518000 & -1.483835000 & 1.406909000 \\
$\mathrm{H}$ & -1.438551000 & 4.081737000 & -1.643978000 \\
$\mathrm{H}$ & -1.548360000 & 1.077255000 & 1.092050000 \\
$\mathrm{O}$ & -0.028114000 & -0.998788000 & 1.629178000 \\
$\mathrm{C}$ & -1.827013000 & 0.153345000 & 0.588991000 \\
$\mathrm{H}$ & -2.602515000 & -0.739123000 & 2.331405000 \\
$\mathrm{C}$ & -0.567074000 & -0.740965000 & 0.520021000 \\
$\mathrm{C}$ & -2.988259000 & -0.510057000 & 1.334717000 \\
$\mathrm{H}$ & -3.797551000 & 0.212671000 & 1.472281000 \\
$\mathrm{H}$ & -2.018180000 & 1.492788000 & -1.042135000 \\
$\mathrm{~N}$ & -2.150288000 & 0.496740000 & -0.844462000 \\
$\mathrm{O}$ & -0.192907000 & -1.103346000 & -0.612456000 \\
$\mathrm{H}$ & -1.434506000 & -0.047468000 & -1.378828000 \\
$\mathrm{C}$ & -3.525521000 & -1.797961000 & 0.705399000 \\
$\mathrm{H}$ & -3.093839000 & 0.157592000 & -1.114022000 \\
$\mathrm{H}$ & -5.910407000 & 0.034878000 & 0.425881000 \\
$\mathrm{H}$ & -4.148486000 & -2.337181000 & 1.420264000 \\
$\mathrm{H}$ & -2.712991000 & -2.468193000 & 0.417119000 \\
$\mathrm{C}$ & -6.071950000 & -0.899315000 & -0.111875000 \\
$\mathrm{~S}$ & -4.524208000 & -1.556896000 & -0.825000000 \\
$\mathrm{H}$ & -6.518537000 & -1.642196000 & 0.549009000 \\
$\mathrm{H}$ & -6.746611000 & -0.716061000 & -0.947566000
\end{tabular}


TABLE S34: Geometry for WM-Z3.

\begin{tabular}{lrrr} 
Element & $\mathrm{X}$ & $\mathrm{Y}$ & $\mathrm{Z}$ \\
\hline $\mathrm{C}$ & 2.464399000 & 1.149809000 & 0.595356000 \\
$\mathrm{C}$ & 3.560910000 & 0.210750000 & 0.203822000 \\
$\mathrm{H}$ & 4.187274000 & 0.635688000 & -0.583795000 \\
$\mathrm{H}$ & 2.695768000 & 1.356246000 & 2.795883000 \\
$\mathrm{H}$ & 0.723422000 & 2.887435000 & 2.665905000 \\
$\mathrm{C}$ & 2.191910000 & 1.585013000 & 1.869514000 \\
$\mathrm{~N}$ & 1.115505000 & 2.441111000 & 1.852817000 \\
$\mathrm{H}$ & 2.479476000 & -0.973795000 & -1.252020000 \\
$\mathrm{C}$ & 0.660324000 & 2.579605000 & 0.559665000 \\
$\mathrm{H}$ & -0.987882000 & 3.983903000 & 0.680766000 \\
$\mathrm{O}$ & 4.988479000 & -1.686115000 & -1.517465000 \\
$\mathrm{C}$ & 3.025294000 & -1.140594000 & -0.321739000 \\
$\mathrm{C}$ & -0.384971000 & 3.347811000 & 0.042827000 \\
$\mathrm{C}$ & 4.139473000 & -2.140881000 & -0.594711000 \\
$\mathrm{H}$ & 5.684890000 & -2.347574000 & -1.660889000 \\
$\mathrm{C}$ & 1.489906000 & 1.770922000 & -0.268162000 \\
$\mathrm{~N}$ & 2.075712000 & -1.775747000 & 0.636116000 \\
$\mathrm{O}$ & 4.226146000 & -3.203926000 & -0.032656000 \\
$\mathrm{C}$ & -0.600033000 & 3.301353000 & -1.331883000 \\
$\mathrm{H}$ & 4.214665000 & 0.025123000 & 1.061510000 \\
$\mathrm{C}$ & 1.247936000 & 1.745243000 & -1.650545000 \\
$\mathrm{H}$ & 2.395805000 & -2.724845000 & 0.858135000 \\
$\mathrm{C}$ & 0.210811000 & 2.507597000 & -2.170594000 \\
$\mathrm{H}$ & 1.873916000 & 1.160875000 & -2.314955000 \\
$\mathrm{H}$ & 0.034304000 & 2.515945000 & -3.239468000 \\
$\mathrm{H}$ & 1.963459000 & -1.239248000 & 1.503135000 \\
$\mathrm{H}$ & 1.070472000 & -1.759540000 & 0.227642000 \\
$\mathrm{H}$ & -1.374984000 & 3.919929000 & -1.770012000 \\
$\mathrm{H}$ & -1.764336000 & 1.219209000 & 1.041878000 \\
$\mathrm{O}$ & -0.270686000 & -0.720092000 & 1.987929000 \\
$\mathrm{C}$ & -1.980211000 & 0.237416000 & 0.627204000 \\
$\mathrm{H}$ & -2.969993000 & -0.460531000 & 2.348662000 \\
$\mathrm{C}$ & -0.736448000 & -0.654553000 & 0.852600000 \\
$\mathrm{C}$ & -3.233242000 & -0.337676000 & 1.294673000 \\
$\mathrm{H}$ & -4.040191000 & 0.399928000 & 1.259365000 \\
$\mathrm{H}$ & -1.894725000 & 1.352017000 & -1.173236000 \\
$\mathrm{~N}$ & -2.123001000 & 0.399916000 & -0.869545000 \\
$\mathrm{O}$ & -0.306689000 & -1.244876000 & -0.203033000 \\
$\mathrm{H}$ & -1.405314000 & -0.253247000 & -1.249331000 \\
$\mathrm{C}$ & -3.717140000 & -1.681213000 & 0.743073000 \\
$\mathrm{H}$ & -3.055637000 & 0.087709000 & -1.201435000 \\
$\mathrm{H}$ & -6.041709000 & 0.112886000 & 0.046315000 \\
$\mathrm{H}$ & -4.419392000 & -2.145117000 & 1.436836000 \\
$\mathrm{H}$ & -2.887722000 & -2.378423000 & 0.609017000 \\
$\mathrm{H}$ & -6.155050000 & -0.865708000 & -0.420049000 \\
& -4.546775000 & -1.586087000 & -0.900422000 \\
& -6.677336000 & -1.545586000 & 0.252998000 \\
-6.734551000 & -0.757851000 & -1.336371000
\end{tabular}


TABLE S35: Geometry for WW-A1.

\begin{tabular}{|c|c|c|c|}
\hline Element & $\mathrm{X}$ & Y & $\mathrm{Z}$ \\
\hline $\mathrm{H}$ & 3.227915000 & 2.831590000 & -0.318813000 \\
\hline $\mathrm{H}$ & 0.185459000 & 0.081300000 & 2.844791000 \\
\hline $\mathrm{H}$ & 4.172470000 & -1.990003000 & 0.622040000 \\
\hline $\mathrm{H}$ & 2.719947000 & -3.312180000 & 2.139334000 \\
\hline $\mathrm{H}$ & 0.762352000 & -2.283015000 & 3.233166000 \\
\hline $\mathrm{H}$ & -2.799803000 & -0.573088000 & -3.262541000 \\
\hline $\mathrm{H}$ & -5.264002000 & 0.763283000 & 0.886164000 \\
\hline $\mathrm{H}$ & -1.314363000 & -2.282697000 & 1.392178000 \\
\hline $\mathrm{H}$ & -2.583203000 & -1.515692000 & 3.364981000 \\
\hline $\mathrm{H}$ & -4.524550000 & -0.012959000 & 3.120977000 \\
\hline $\mathrm{H}$ & 0.756848000 & 4.846216000 & -1.849344000 \\
\hline $\mathrm{O}$ & 0.475284000 & 4.591995000 & -0.956342000 \\
\hline $\mathrm{O}$ & 0.089891000 & 2.629772000 & -1.978458000 \\
\hline $\mathrm{C}$ & 0.068211000 & 3.315606000 & -0.982134000 \\
\hline $\mathrm{H}$ & -1.117165000 & 3.607176000 & 0.748637000 \\
\hline $\mathrm{C}$ & -0.405723000 & 2.854397000 & 0.388485000 \\
\hline $\mathrm{H}$ & -1.888242000 & 1.585206000 & -0.276215000 \\
\hline $\mathrm{N}$ & -1.014662000 & 1.527423000 & 0.240498000 \\
\hline $\mathrm{H}$ & 1.214893000 & 3.808988000 & 1.436865000 \\
\hline $\mathrm{C}$ & 2.889361000 & 1.950722000 & 0.204005000 \\
\hline $\mathrm{C}$ & 0.778439000 & 2.810441000 & 1.388970000 \\
\hline $\mathrm{C}$ & 1.816904000 & 1.786261000 & 1.046395000 \\
\hline $\mathrm{N}$ & 3.574435000 & 0.754127000 & 0.079653000 \\
\hline $\mathrm{H}$ & 4.431131000 & 0.627997000 & -0.435477000 \\
\hline $\mathrm{H}$ & -1.249744000 & 1.132087000 & 1.146914000 \\
\hline $\mathrm{H}$ & 0.364740000 & 2.604621000 & 2.380137000 \\
\hline $\mathrm{C}$ & 1.852815000 & 0.414632000 & 1.494860000 \\
\hline $\mathrm{C}$ & 2.977169000 & -0.200519000 & 0.878946000 \\
\hline $\mathrm{C}$ & 3.306675000 & -1.540957000 & 1.093668000 \\
\hline $\mathrm{C}$ & 1.045543000 & -0.353512000 & 2.349485000 \\
\hline $\mathrm{C}$ & 1.369541000 & -1.684463000 & 2.564978000 \\
\hline $\mathrm{C}$ & 2.489349000 & -2.272382000 & 1.943373000 \\
\hline $\mathrm{C}$ & -2.037224000 & -1.382169000 & -1.337883000 \\
\hline $\mathrm{C}$ & -0.819094000 & -2.160122000 & -1.719137000 \\
\hline $\mathrm{H}$ & -0.878724000 & -3.195077000 & -1.375517000 \\
\hline $\mathrm{H}$ & -4.601021000 & 0.481372000 & -1.867546000 \\
\hline $\mathrm{C}$ & -2.842178000 & -0.665453000 & -2.188128000 \\
\hline $\mathrm{N}$ & -3.821850000 & -0.013741000 & -1.464254000 \\
\hline $\mathrm{H}$ & 0.489599000 & -1.690688000 & -0.039497000 \\
\hline $\mathrm{C}$ & -3.678485000 & -0.320554000 & -0.122389000 \\
\hline $\mathrm{O}$ & 1.724749000 & -3.523767000 & -1.503426000 \\
\hline $\mathrm{C}$ & 0.474438000 & -1.559683000 & -1.122839000 \\
\hline $\mathrm{C}$ & -4.403401000 & 0.112939000 & 0.989368000 \\
\hline $\mathrm{C}$ & 1.727367000 & -2.201405000 & -1.693358000 \\
\hline $\mathrm{H}$ & 2.533790000 & -3.896763000 & -1.888279000 \\
\hline $\mathrm{C}$ & -2.553545000 & -1.180607000 & -0.006294000 \\
\hline $\mathrm{N}$ & 0.550034000 & -0.095290000 & -1.376491000 \\
\hline $\mathrm{O}$ & 2.598925000 & -1.585657000 & -2.255686000 \\
\hline $\mathrm{C}$ & -3.981852000 & -0.325307000 & 2.237298000 \\
\hline $\mathrm{H}$ & -0.727781000 & -2.195892000 & -2.809418000 \\
\hline $\mathrm{C}$ & -2.158523000 & -1.616019000 & 1.268207000 \\
\hline $\mathrm{H}$ & 0.241054000 & 0.155932000 & -2.316795000 \\
\hline $\mathrm{C}$ & -2.871341000 & -1.181289000 & 2.375621000 \\
\hline $\mathrm{H}$ & -0.061344000 & 0.482051000 & -0.697614000 \\
\hline $\mathrm{H}$ & 1.516573000 & 0.230501000 & -1.292663000 \\
\hline
\end{tabular}


TABLE S36: Geometry for WW-A2.

\begin{tabular}{|c|c|c|c|}
\hline Element & $\mathrm{X}$ & $\mathrm{Y}$ & $\mathrm{Z}$ \\
\hline $\mathrm{H}$ & 2.794616000 & -1.780661000 & -1.499086000 \\
\hline $\mathrm{H}$ & 1.996476000 & -1.694683000 & -3.847529000 \\
\hline $\mathrm{H}$ & -0.391113000 & -2.310798000 & 2.307050000 \\
\hline $\mathrm{H}$ & -0.399124000 & -1.964401000 & -4.379613000 \\
\hline $\mathrm{H}$ & -2.065280000 & -2.306478000 & -2.593992000 \\
\hline $\mathrm{H}$ & 3.122346000 & 1.897934000 & -2.338651000 \\
\hline $\mathrm{H}$ & 4.896776000 & -1.126689000 & 1.222708000 \\
\hline $\mathrm{H}$ & 0.775075000 & 1.525397000 & 2.274253000 \\
\hline $\mathrm{H}$ & 1.751722000 & -0.001960000 & 3.937549000 \\
\hline $\mathrm{H}$ & 3.771739000 & -1.324324000 & 3.423803000 \\
\hline $\mathrm{C}$ & 1.983212000 & 1.827320000 & -0.433466000 \\
\hline $\mathrm{C}$ & 0.852423000 & 2.782433000 & -0.656109000 \\
\hline $\mathrm{H}$ & 0.920156000 & 3.642670000 & 0.015037000 \\
\hline $\mathrm{H}$ & 4.693345000 & 0.290497000 & -1.225939000 \\
\hline $\mathrm{C}$ & 2.970884000 & 1.523980000 & -1.338036000 \\
\hline $\mathrm{N}$ & 3.849696000 & 0.619145000 & -0.784937000 \\
\hline $\mathrm{H}$ & -0.632914000 & 1.870293000 & 0.645689000 \\
\hline $\mathrm{C}$ & 3.444488000 & 0.311812000 & 0.498138000 \\
\hline $\mathrm{O}$ & -1.657221000 & 4.168395000 & -0.051695000 \\
\hline $\mathrm{C}$ & -0.523221000 & 2.134895000 & -0.403381000 \\
\hline $\mathrm{C}$ & 3.996571000 & -0.562276000 & 1.435909000 \\
\hline $\mathrm{C}$ & -1.682531000 & 3.041808000 & -0.766708000 \\
\hline $\mathrm{H}$ & -2.407577000 & 4.725677000 & -0.313932000 \\
\hline $\mathrm{C}$ & 2.265544000 & 1.064225000 & 0.760598000 \\
\hline $\mathrm{N}$ & -0.693568000 & 0.867402000 & -1.166092000 \\
\hline $\mathrm{O}$ & -2.510447000 & 2.770370000 & -1.602154000 \\
\hline $\mathrm{C}$ & 3.360087000 & -0.667012000 & 2.667685000 \\
\hline $\mathrm{H}$ & 0.894134000 & 3.175921000 & -1.676659000 \\
\hline $\mathrm{C}$ & 1.655420000 & 0.947369000 & 2.020487000 \\
\hline $\mathrm{H}$ & -0.698042000 & 1.032846000 & -2.174073000 \\
\hline $\mathrm{C}$ & 2.206073000 & 0.086307000 & 2.958208000 \\
\hline $\mathrm{H}$ & 0.075047000 & 0.218201000 & -0.965532000 \\
\hline $\mathrm{H}$ & -1.628206000 & 0.386549000 & -0.926025000 \\
\hline $\mathrm{H}$ & -3.746478000 & 0.662122000 & -0.599314000 \\
\hline $\mathrm{H}$ & -3.572932000 & -0.725454000 & -1.436426000 \\
\hline $\mathrm{N}$ & -3.222326000 & -0.209244000 & -0.634509000 \\
\hline $\mathrm{O}$ & -2.334786000 & 0.866368000 & 1.681011000 \\
\hline $\mathrm{C}$ & 1.743109000 & -1.880004000 & -1.731157000 \\
\hline $\mathrm{C}$ & 1.286767000 & -1.830294000 & -3.040522000 \\
\hline $\mathrm{C}$ & -3.433519000 & -0.997968000 & 0.588807000 \\
\hline $\mathrm{N}$ & 0.941642000 & -2.118729000 & 0.649069000 \\
\hline $\mathrm{C}$ & 0.795967000 & -2.062995000 & -0.719563000 \\
\hline $\mathrm{C}$ & -2.883425000 & -0.208318000 & 1.764152000 \\
\hline $\mathrm{H}$ & 1.804885000 & -1.974742000 & 1.153929000 \\
\hline $\mathrm{C}$ & -0.082149000 & -1.979396000 & -3.343841000 \\
\hline $\mathrm{C}$ & -0.297201000 & -2.254857000 & 1.234110000 \\
\hline $\mathrm{C}$ & -0.594110000 & -2.199513000 & -1.001174000 \\
\hline $\mathrm{H}$ & -4.498654000 & -1.162690000 & 0.796273000 \\
\hline $\mathrm{C}$ & -1.020233000 & -2.169937000 & -2.340133000 \\
\hline $\mathrm{C}$ & -1.272475000 & -2.307352000 & 0.265151000 \\
\hline $\mathrm{C}$ & -2.751593000 & -2.385603000 & 0.484681000 \\
\hline $\mathrm{H}$ & -3.230116000 & -2.929887000 & -0.335004000 \\
\hline $\mathrm{O}$ & -3.089775000 & -0.842732000 & 2.925980000 \\
\hline $\mathrm{H}$ & -2.756760000 & -0.284308000 & 3.645730000 \\
\hline $\mathrm{H}$ & -2.979930000 & -2.938010000 & 1.396886000 \\
\hline
\end{tabular}


TABLE S37: Geometry for WW-A3.

\begin{tabular}{|c|c|c|c|}
\hline Element & $\mathrm{X}$ & Y & $\mathrm{Z}$ \\
\hline $\mathrm{H}$ & -0.449873000 & 4.677462000 & -1.782309000 \\
\hline $\mathrm{O}$ & -0.514517000 & 4.440435000 & -0.843681000 \\
\hline $\mathrm{O}$ & -0.642914000 & 2.370257000 & -1.703351000 \\
\hline $\mathrm{C}$ & -0.639389000 & 3.109544000 & -0.746347000 \\
\hline $\mathrm{H}$ & -1.498349000 & 3.321305000 & 1.176939000 \\
\hline $\mathrm{C}$ & -0.753523000 & 2.669436000 & 0.705415000 \\
\hline $\mathrm{H}$ & -2.086433000 & 1.139839000 & 0.356182000 \\
\hline $\mathrm{H}$ & 2.635272000 & 3.312183000 & -0.689427000 \\
\hline $\mathrm{N}$ & -1.147703000 & 1.255677000 & 0.732339000 \\
\hline $\mathrm{H}$ & 0.835868000 & 3.949224000 & 1.390478000 \\
\hline $\mathrm{C}$ & 2.575804000 & 2.399682000 & -0.116975000 \\
\hline $\mathrm{C}$ & 0.594490000 & 2.886457000 & 1.441379000 \\
\hline $\mathrm{C}$ & 1.723224000 & 2.058999000 & 0.905545000 \\
\hline $\mathrm{N}$ & 3.453140000 & 1.359433000 & -0.362321000 \\
\hline $\mathrm{H}$ & 4.194860000 & 1.375518000 & -1.043868000 \\
\hline $\mathrm{H}$ & -1.175308000 & 0.917646000 & 1.689296000 \\
\hline $\mathrm{H}$ & 0.432663000 & 2.657306000 & 2.498722000 \\
\hline $\mathrm{C}$ & 2.106836000 & 0.735333000 & 1.334416000 \\
\hline $\mathrm{C}$ & 3.202884000 & 0.330756000 & 0.522195000 \\
\hline $\mathrm{C}$ & 3.821970000 & -0.913295000 & 0.667678000 \\
\hline $\mathrm{C}$ & 1.631898000 & -0.149683000 & 2.316203000 \\
\hline $\mathrm{H}$ & 0.810727000 & 0.130085000 & 2.966115000 \\
\hline $\mathrm{C}$ & 2.243285000 & -1.386232000 & 2.462935000 \\
\hline $\mathrm{C}$ & 3.328790000 & -1.763884000 & 1.646758000 \\
\hline $\mathrm{H}$ & 4.662670000 & -1.199783000 & 0.047151000 \\
\hline $\mathrm{H}$ & 3.791017000 & -2.732330000 & 1.792311000 \\
\hline $\mathrm{H}$ & 1.894599000 & -2.070112000 & 3.227122000 \\
\hline $\mathrm{H}$ & -1.053998000 & -3.416433000 & 1.692971000 \\
\hline $\mathrm{H}$ & -3.102606000 & -2.368072000 & 2.695971000 \\
\hline $\mathrm{C}$ & -1.670026000 & -2.705078000 & 1.165217000 \\
\hline $\mathrm{N}$ & -2.755229000 & -2.118697000 & 1.784036000 \\
\hline $\mathrm{C}$ & -3.384927000 & -1.267890000 & 0.896360000 \\
\hline $\mathrm{H}$ & -5.042032000 & -0.407240000 & 1.999596000 \\
\hline $\mathrm{C}$ & -1.561285000 & -2.236395000 & -0.120737000 \\
\hline $\mathrm{H}$ & -0.086289000 & -3.556225000 & -0.944584000 \\
\hline $\mathrm{O}$ & 2.265507000 & -3.168292000 & -1.802491000 \\
\hline $\mathrm{C}$ & 0.737161000 & -1.589409000 & -0.929485000 \\
\hline $\mathrm{C}$ & -4.493729000 & -0.436325000 & 1.065364000 \\
\hline $\mathrm{C}$ & -0.462676000 & -2.543238000 & -1.089885000 \\
\hline $\mathrm{H}$ & 3.005713000 & -3.311590000 & -2.413614000 \\
\hline $\mathrm{C}$ & -2.656129000 & -1.321748000 & -0.323341000 \\
\hline $\mathrm{C}$ & 1.891127000 & -1.887515000 & -1.874380000 \\
\hline $\mathrm{C}$ & -4.872938000 & 0.353474000 & -0.011917000 \\
\hline $\mathrm{H}$ & -0.832179000 & -2.494073000 & -2.119852000 \\
\hline $\mathrm{C}$ & -3.076414000 & -0.524501000 & -1.400340000 \\
\hline $\mathrm{H}$ & -5.730993000 & 1.007140000 & 0.084270000 \\
\hline $\mathrm{C}$ & -4.172852000 & 0.308444000 & -1.234295000 \\
\hline $\mathrm{H}$ & -2.564829000 & -0.556990000 & -2.356383000 \\
\hline $\mathrm{H}$ & -4.506600000 & 0.926313000 & -2.058660000 \\
\hline $\mathrm{O}$ & 2.401582000 & -1.060130000 & -2.588654000 \\
\hline $\mathrm{H}$ & 1.117908000 & -1.663320000 & 0.090864000 \\
\hline $\mathrm{H}$ & -0.212674000 & 0.271109000 & -0.328956000 \\
\hline $\mathrm{N}$ & 0.343405000 & -0.168309000 & -1.139504000 \\
\hline $\mathrm{H}$ & 1.174492000 & 0.411289000 & -1.281747000 \\
\hline $\mathrm{H}$ & -0.227673000 & -0.036736000 & -1.975596000 \\
\hline
\end{tabular}


TABLE S38: Geometry for WW-A4.

\begin{tabular}{|c|c|c|c|}
\hline Element & $\mathrm{X}$ & $\mathrm{Y}$ & Z \\
\hline $\mathrm{H}$ & 4.450288000 & -0.444753000 & 2.314966000 \\
\hline $\mathrm{H}$ & 5.108713000 & -1.970604000 & 0.474318000 \\
\hline $\mathrm{H}$ & 4.197316000 & -1.683098000 & -1.799353000 \\
\hline $\mathrm{H}$ & 2.569642000 & 0.105299000 & -2.301845000 \\
\hline $\mathrm{H}$ & 1.191350000 & 3.274569000 & 1.553062000 \\
\hline $\mathrm{H}$ & 0.520958000 & -1.467004000 & 2.628255000 \\
\hline $\mathrm{H}$ & 1.687303000 & -2.655065000 & -2.084763000 \\
\hline $\mathrm{H}$ & -0.075638000 & -3.106842000 & -3.771008000 \\
\hline $\mathrm{H}$ & -2.463069000 & -3.074942000 & -3.147753000 \\
\hline $\mathrm{H}$ & -3.164694000 & -2.591316000 & -0.835626000 \\
\hline $\mathrm{N}$ & -0.810969000 & 0.623849000 & -0.827527000 \\
\hline $\mathrm{C}$ & -0.686733000 & 2.081915000 & -1.100699000 \\
\hline $\mathrm{C}$ & -1.530299000 & 2.384223000 & -2.324786000 \\
\hline $\mathrm{C}$ & 0.792528000 & 2.493319000 & -1.304404000 \\
\hline $\mathrm{O}$ & -1.681432000 & 3.700995000 & -2.496619000 \\
\hline $\mathrm{O}$ & -1.974329000 & 1.533085000 & -3.053980000 \\
\hline $\mathrm{C}$ & 1.666516000 & 1.987597000 & -0.197399000 \\
\hline $\mathrm{C}$ & 1.716783000 & 2.451625000 & 1.095327000 \\
\hline $\mathrm{N}$ & 2.579280000 & 1.672402000 & 1.834826000 \\
\hline $\mathrm{C}$ & 3.122303000 & 0.691202000 & 1.029311000 \\
\hline $\mathrm{C}$ & 2.565276000 & 0.860803000 & -0.268050000 \\
\hline $\mathrm{C}$ & 4.030440000 & -0.327359000 & 1.322887000 \\
\hline $\mathrm{C}$ & 4.395022000 & -1.178154000 & 0.285692000 \\
\hline $\mathrm{C}$ & 3.871399000 & -1.013860000 & -1.012431000 \\
\hline $\mathrm{C}$ & 2.961163000 & -0.007149000 & -1.297417000 \\
\hline $\mathrm{H}$ & -0.815871000 & 0.081357000 & -1.696983000 \\
\hline $\mathrm{H}$ & -1.718447000 & 0.408850000 & -0.304467000 \\
\hline $\mathrm{H}$ & -0.030830000 & 0.311298000 & -0.241532000 \\
\hline $\mathrm{H}$ & -1.088938000 & 2.596757000 & -0.229155000 \\
\hline $\mathrm{H}$ & 1.147212000 & 2.106492000 & -2.263869000 \\
\hline $\mathrm{H}$ & 0.816748000 & 3.581800000 & -1.376855000 \\
\hline $\mathrm{H}$ & -2.186587000 & 3.856723000 & -3.311154000 \\
\hline $\mathrm{H}$ & 2.853939000 & 1.861183000 & 2.785184000 \\
\hline $\mathrm{N}$ & -3.205889000 & 0.180248000 & 0.592080000 \\
\hline $\mathrm{C}$ & -2.946933000 & -0.213887000 & 1.987224000 \\
\hline $\mathrm{C}$ & -1.950881000 & 0.768369000 & 2.582484000 \\
\hline $\mathrm{C}$ & -2.412364000 & -1.666825000 & 2.072503000 \\
\hline $\mathrm{O}$ & -1.748197000 & 0.528764000 & 3.886017000 \\
\hline $\mathrm{O}$ & -1.405479000 & 1.662693000 & 1.977983000 \\
\hline $\mathrm{C}$ & -1.177960000 & -1.905998000 & 1.258941000 \\
\hline $\mathrm{C}$ & 0.127200000 & -1.737355000 & 1.661085000 \\
\hline $\mathrm{N}$ & 0.978460000 & -1.988086000 & 0.604424000 \\
\hline $\mathrm{C}$ & 0.238723000 & -2.336227000 & -0.506783000 \\
\hline $\mathrm{C}$ & -1.131668000 & -2.298512000 & -0.127627000 \\
\hline $\mathrm{C}$ & 0.640579000 & -2.625233000 & -1.814310000 \\
\hline $\mathrm{C}$ & -0.352877000 & -2.877178000 & -2.749560000 \\
\hline $\mathrm{C}$ & -1.716963000 & -2.857790000 & -2.393602000 \\
\hline $\mathrm{C}$ & -2.111823000 & -2.578785000 & -1.095136000 \\
\hline $\mathrm{H}$ & -3.726129000 & 1.053651000 & 0.566033000 \\
\hline $\mathrm{H}$ & -3.786851000 & -0.519119000 & 0.138841000 \\
\hline $\mathrm{H}$ & -3.849314000 & -0.155678000 & 2.608395000 \\
\hline $\mathrm{H}$ & -2.237279000 & -1.893259000 & 3.124985000 \\
\hline $\mathrm{H}$ & -3.214643000 & -2.333327000 & 1.742624000 \\
\hline $\mathrm{H}$ & -1.139200000 & 1.198837000 & 4.232350000 \\
\hline $\mathrm{H}$ & 1.986770000 & -1.933463000 & 0.642021000 \\
\hline
\end{tabular}


TABLE S39: Geometry for WW-A5.

\begin{tabular}{lrrr} 
Element & $\mathrm{X}$ & $\mathrm{Y}$ & $\mathrm{Z}$ \\
\hline $\mathrm{C}$ & 0.296571000 & -2.820169000 & -0.309730000 \\
$\mathrm{C}$ & -0.430324000 & -0.707299000 & 2.479129000 \\
$\mathrm{C}$ & -0.397959000 & -2.839432000 & 1.082678000 \\
$\mathrm{C}$ & -1.527295000 & 2.693047000 & -0.970992000 \\
$\mathrm{C}$ & -0.684801000 & -2.936877000 & -1.467728000 \\
$\mathrm{C}$ & -0.972191000 & -1.527070000 & 1.521328000 \\
$\mathrm{C}$ & -2.279234000 & 0.358984000 & 1.756892000 \\
$\mathrm{C}$ & -2.165230000 & -0.866958000 & 1.043931000 \\
$\mathrm{C}$ & -3.324627000 & 1.257725000 & 1.536614000 \\
$\mathrm{C}$ & -3.143948000 & -1.181076000 & 0.083755000 \\
$\mathrm{C}$ & -4.266270000 & 0.923520000 & 0.573959000 \\
$\mathrm{C}$ & -4.176301000 & -0.282402000 & -0.146409000 \\
$\mathrm{C}$ & -0.333595000 & 1.799249000 & -0.686075000 \\
$\mathrm{C}$ & 0.967013000 & 2.452788000 & -1.210062000 \\
$\mathrm{C}$ & 2.151850000 & 1.548521000 & -1.111051000 \\
$\mathrm{C}$ & 2.471923000 & 1.201792000 & 1.465569000 \\
$\mathrm{C}$ & 2.744422000 & 1.034022000 & 0.098939000 \\
$\mathrm{C}$ & 2.853814000 & 1.006837000 & -2.158318000 \\
$\mathrm{C}$ & 3.243651000 & 0.516529000 & 2.392200000 \\
$\mathrm{C}$ & 3.805914000 & 0.174124000 & -0.291214000 \\
$\mathrm{C}$ & 4.286768000 & -0.336552000 & 1.982350000 \\
$\mathrm{C}$ & 4.584005000 & -0.517902000 & 0.638328000 \\
$\mathrm{H}$ & 0.355585000 & -3.151400000 & 1.809976000 \\
$\mathrm{H}$ & -0.536771000 & 0.548936000 & -2.356613000 \\
$\mathrm{H}$ & -1.086093000 & 1.119249000 & 3.345156000 \\
$\mathrm{H}$ & -2.279657000 & 4.332036000 & -0.410816000 \\
$\mathrm{O}$ & -1.533783000 & 3.762234000 & -0.163332000 \\
$\mathrm{O}$ & -0.848262000 & -2.105011000 & -2.330251000 \\
$\mathrm{O}$ & -2.327596000 & 2.476814000 & -1.842489000 \\
$\mathrm{O}$ & -1.345797000 & -4.102814000 & -1.433107000 \\
$\mathrm{H}$ & -1.508174000 & 0.128322000 & -1.107018000 \\
$\mathrm{H}$ & -1.159608000 & -3.621667000 & 1.072286000 \\
$\mathrm{H}$ & -1.930530000 & -4.151884000 & -2.205753000 \\
$\mathrm{H}$ & 0.125666000 & -0.270040000 & -1.019580000 \\
$\mathrm{H}$ & -0.260918000 & 1.610586000 & 0.384191000 \\
$\mathrm{H}$ & 0.915260000 & -3.724105000 & -0.361940000 \\
$\mathrm{H}$ & 1.558561000 & -1.348456000 & 0.379983000 \\
$\mathrm{H}$ & 0.814891000 & 2.758347000 & -2.250072000 \\
$\mathrm{H}$ & 1.801876000 & -1.714456000 & -1.191238000 \\
$\mathrm{H}$ & & &
\end{tabular}


TABLE S40: Geometry for WW-A6.

\begin{tabular}{lrrr} 
Element & $\mathrm{X}$ & $\mathrm{Y}$ & $\mathrm{Z}$ \\
\hline $\mathrm{C}$ & 0.063348000 & 2.085710000 & -0.262157000 \\
$\mathrm{C}$ & 0.518670000 & -1.676009000 & 1.702862000 \\
$\mathrm{C}$ & 0.956950000 & -2.756277000 & -0.434712000 \\
$\mathrm{C}$ & 1.388800000 & 2.463327000 & -0.974376000 \\
$\mathrm{C}$ & 1.410070000 & -2.241907000 & 0.770736000 \\
$\mathrm{C}$ & 2.378501000 & 1.345884000 & -1.022110000 \\
$\mathrm{C}$ & 2.824062000 & 0.710207000 & -2.157772000 \\
$\mathrm{C}$ & 3.059945000 & 0.958199000 & 1.477351000 \\
$\mathrm{C}$ & 3.073714000 & 0.746805000 & 0.090083000 \\
$\mathrm{C}$ & 3.890700000 & 0.191619000 & 2.279676000 \\
$\mathrm{C}$ & 3.938397000 & -0.242608000 & -0.447763000 \\
$\mathrm{C}$ & 4.744348000 & -0.780957000 & 1.723593000 \\
$\mathrm{C}$ & 4.782872000 & -1.010151000 & 0.355330000 \\
$\mathrm{C}$ & -0.413550000 & -2.681248000 & -0.698009000 \\
$\mathrm{C}$ & -0.842961000 & -1.607678000 & 1.443845000 \\
$\mathrm{C}$ & -0.965683000 & 3.168641000 & -0.523314000 \\
$\mathrm{C}$ & -1.331779000 & -2.097464000 & 0.219046000 \\
$\mathrm{C}$ & -2.468590000 & -2.751147000 & -1.615856000 \\
$\mathrm{C}$ & -2.633981000 & -2.136606000 & -0.399454000 \\
$\mathrm{C}$ & -3.304181000 & 0.626413000 & 1.340581000 \\
$\mathrm{C}$ & -3.911127000 & -1.544994000 & 0.108798000 \\
$\mathrm{C}$ & -3.933804000 & 0.014906000 & 0.098054000 \\
$\mathrm{H}$ & 0.018109000 & 0.499896000 & -1.622285000 \\
$\mathrm{H}$ & 0.214688000 & 1.988161000 & 0.809847000 \\
$\mathrm{H}$ & 1.154630000 & 2.789387000 & -1.992808000 \\
$\mathrm{H}$ & 1.795102000 & 3.331247000 & -0.449079000 \\
$\mathrm{O}$ & -0.747612000 & 4.220505000 & 0.270851000 \\
$\mathrm{O}$ & -1.813147000 & 3.116170000 & -1.379657000 \\
$\mathrm{H}$ & -2.351129000 & 1.372029000 & 1.348974000 \\
$\mathrm{O}$ & -3.956070000 & 0.257139000 & 2.451906000 \\
$\mathrm{H}$ & & &
\end{tabular}


TABLE S41: Geometry for WW-B1.

\begin{tabular}{|c|c|c|c|}
\hline Element & $\mathrm{X}$ & $\mathrm{Y}$ & $\mathrm{Z}$ \\
\hline $\mathrm{C}$ & -0.267950000 & 1.350711000 & 2.514458000 \\
\hline $\mathrm{C}$ & -0.585051000 & -1.406752000 & -0.302908000 \\
\hline $\mathrm{C}$ & -0.605230000 & 2.305668000 & -0.056367000 \\
\hline $\mathrm{C}$ & -1.499643000 & 1.167653000 & 1.900283000 \\
\hline $\mathrm{C}$ & -1.685380000 & 1.650750000 & 0.596012000 \\
\hline $\mathrm{C}$ & -2.377225000 & 2.331626000 & -1.444549000 \\
\hline $\mathrm{C}$ & -2.805115000 & 1.681038000 & -0.312370000 \\
\hline $\mathrm{C}$ & -4.160343000 & 1.096371000 & -0.073870000 \\
\hline $\mathrm{C}$ & -4.160701000 & -0.443448000 & -0.167885000 \\
\hline $\mathrm{C}$ & -5.528936000 & -1.077781000 & 0.039480000 \\
\hline $\mathrm{C}$ & 0.638261000 & 2.478762000 & 0.552903000 \\
\hline $\mathrm{C}$ & 0.791579000 & 1.994026000 & 1.845019000 \\
\hline $\mathrm{C}$ & 0.853234000 & -1.196509000 & 0.133123000 \\
\hline $\mathrm{C}$ & 1.814484000 & -1.959301000 & -0.814021000 \\
\hline $\mathrm{C}$ & 3.237620000 & -1.702264000 & -0.445412000 \\
\hline $\mathrm{C}$ & 3.544473000 & 0.839652000 & -0.988429000 \\
\hline $\mathrm{C}$ & 3.913307000 & -0.431170000 & -0.520857000 \\
\hline $\mathrm{C}$ & 4.132526000 & -2.593821000 & 0.094703000 \\
\hline $\mathrm{C}$ & 4.465881000 & 1.873250000 & -0.919165000 \\
\hline $\mathrm{C}$ & 5.221725000 & -0.623114000 & -0.004565000 \\
\hline $\mathrm{C}$ & 5.755015000 & 1.662907000 & -0.392555000 \\
\hline $\mathrm{C}$ & 6.151903000 & 0.415253000 & 0.066918000 \\
\hline $\mathrm{H}$ & -0.529083000 & 3.230366000 & -1.971869000 \\
\hline $\mathrm{H}$ & -0.701414000 & -2.220710000 & 1.333013000 \\
\hline $\mathrm{H}$ & -2.598225000 & -0.997158000 & -1.507200000 \\
\hline $\mathrm{H}$ & -3.474487000 & -0.863965000 & 0.570175000 \\
\hline $\mathrm{H}$ & -3.919483000 & -0.222431000 & -2.234612000 \\
\hline $\mathrm{H}$ & -4.121651000 & -1.784543000 & -1.747286000 \\
\hline $\mathrm{H}$ & -4.533191000 & 1.356980000 & 0.918844000 \\
\hline $\mathrm{H}$ & -4.883563000 & 1.496543000 & -0.792894000 \\
\hline $\mathrm{H}$ & -6.936271000 & -1.134777000 & 1.297581000 \\
\hline $\mathrm{H}$ & 0.987028000 & -0.858420000 & 2.157884000 \\
\hline $\mathrm{H}$ & 1.051309000 & -0.126567000 & 0.043686000 \\
\hline $\mathrm{H}$ & 1.593363000 & -3.030243000 & -0.763300000 \\
\hline $\mathrm{H}$ & 1.601527000 & -1.634322000 & -1.835543000 \\
\hline $\mathrm{H}$ & 1.823768000 & -2.177808000 & 1.662264000 \\
\hline $\mathrm{H}$ & 6.153963000 & -2.394428000 & 0.720550000 \\
\hline $\mathrm{H}$ & -0.124340000 & 1.014869000 & 3.535080000 \\
\hline $\mathrm{H}$ & -2.308228000 & 0.683011000 & 2.436037000 \\
\hline $\mathrm{H}$ & -2.922403000 & 2.591236000 & -2.339500000 \\
\hline $\mathrm{H}$ & 1.455014000 & 2.979238000 & 0.048077000 \\
\hline $\mathrm{H}$ & 1.741609000 & 2.124858000 & 2.348515000 \\
\hline $\mathrm{H}$ & 2.561570000 & 1.007450000 & -1.413619000 \\
\hline $\mathrm{H}$ & 4.019230000 & -3.648641000 & 0.293397000 \\
\hline $\mathrm{H}$ & 4.202281000 & 2.858360000 & -1.286086000 \\
\hline $\mathrm{H}$ & 6.454110000 & 2.489339000 & -0.354671000 \\
\hline $\mathrm{H}$ & 7.148786000 & 0.255968000 & 0.460575000 \\
\hline $\mathrm{N}$ & -1.057273000 & 2.697871000 & -1.299939000 \\
\hline $\mathrm{N}$ & -3.666944000 & -0.895761000 & -1.509427000 \\
\hline $\mathrm{N}$ & 0.966330000 & -1.650613000 & 1.524136000 \\
\hline $\mathrm{N}$ & 5.324590000 & -1.950859000 & 0.362050000 \\
\hline $\mathrm{O}$ & -1.031870000 & -1.019807000 & -1.375451000 \\
\hline $\mathrm{O}$ & -1.337500000 & -2.054010000 & 0.570216000 \\
\hline $\mathrm{O}$ & -6.026253000 & -1.840424000 & -0.751680000 \\
\hline $\mathrm{O}$ & -6.076219000 & -0.695722000 & 1.192352000 \\
\hline
\end{tabular}


TABLE S42: Geometry for WW-CS1.

\begin{tabular}{lrrr} 
Element & $\mathrm{X}$ & $\mathrm{Y}$ & $\mathrm{Z}$ \\
\hline $\mathrm{C}$ & 0.283118000 & -3.011061000 & -0.035600000 \\
$\mathrm{C}$ & 0.496022000 & 2.325739000 & 1.205530000 \\
$\mathrm{C}$ & 0.850752000 & 3.150939000 & -0.026140000 \\
$\mathrm{C}$ & 1.707481000 & 1.828328000 & 2.040500000 \\
$\mathrm{C}$ & 2.266781000 & 0.511118000 & 1.600190000 \\
$\mathrm{C}$ & 2.294810000 & -0.621092000 & 2.379620000 \\
$\mathrm{C}$ & 2.861740000 & 0.153588000 & 0.332800000 \\
$\mathrm{C}$ & 3.115551000 & 0.835808000 & -0.868440000 \\
$\mathrm{C}$ & 3.218939000 & -1.221673000 & 0.420960000 \\
$\mathrm{C}$ & 3.660350000 & 0.142247000 & -1.939570000 \\
$\mathrm{C}$ & 3.774619000 & -1.921283000 & -0.651100000 \\
$\mathrm{C}$ & 3.980589000 & -1.225553000 & -1.834740000 \\
$\mathrm{C}$ & -0.302441000 & -1.943500000 & -0.718950000 \\
$\mathrm{C}$ & -0.543162000 & -3.799880000 & 0.750330000 \\
$\mathrm{C}$ & -0.751690000 & -0.198950000 & -2.077560000 \\
$\mathrm{C}$ & -1.694491000 & -1.671969000 & -0.645010000 \\
$\mathrm{C}$ & -1.924652000 & -3.542549000 & 0.847210000 \\
$\mathrm{C}$ & -1.955890000 & -0.551529000 & -1.518110000 \\
$\mathrm{C}$ & -2.506871000 & -2.494379000 & 0.153580000 \\
$\mathrm{C}$ & -3.278190000 & 0.114742000 & -1.738940000 \\
$\mathrm{C}$ & -3.640729000 & 1.192462000 & -0.680100000 \\
$\mathrm{C}$ & -3.815769000 & 0.587772000 & 0.706240000 \\
$\mathrm{H}$ & 0.149801000 & 0.524970000 & 0.207430000 \\
$\mathrm{H}$ & 1.213010000 & -0.998801000 & -1.890060000 \\
$\mathrm{H}$ & 1.381821000 & 1.738949000 & 3.079130000 \\
$\mathrm{H}$ & 1.858193000 & 4.677638000 & -0.505040000 \\
$\mathrm{O}$ & & &
\end{tabular}


TABLE S43: Geometry for WW-CS4.

\begin{tabular}{lrrr} 
Element & $\mathrm{X}$ & $\mathrm{Y}$ & $\mathrm{Z}$ \\
\hline $\mathrm{C}$ & 0.041110000 & 2.926211000 & -1.003000000 \\
$\mathrm{C}$ & 0.468260000 & -1.847589000 & -0.415510000 \\
$\mathrm{C}$ & 0.598650000 & 2.743111000 & 1.493910000 \\
$\mathrm{C}$ & 1.311350000 & 0.587531000 & 2.656900000 \\
$\mathrm{C}$ & 1.416290000 & 1.494921000 & 1.632500000 \\
$\mathrm{C}$ & 1.558560000 & -2.533489000 & -1.222650000 \\
$\mathrm{C}$ & 2.454990000 & 1.003341000 & 0.756370000 \\
$\mathrm{C}$ & 2.928820000 & -0.217859000 & 1.311360000 \\
$\mathrm{C}$ & 3.029210000 & 1.465501000 & -0.441970000 \\
$\mathrm{C}$ & 3.934210000 & -0.974759000 & 0.706290000 \\
$\mathrm{C}$ & 4.020590000 & 0.709521000 & -1.050370000 \\
$\mathrm{C}$ & 4.468370000 & -0.497889000 & -0.481830000 \\
$\mathrm{C}$ & -0.505030000 & 2.697891000 & 0.399040000 \\
$\mathrm{C}$ & -0.838430000 & -2.672699000 & -0.445270000 \\
$\mathrm{C}$ & -1.966220000 & -2.015159000 & 0.284420000 \\
$\mathrm{C}$ & -2.259260000 & -2.129599000 & 1.620980000 \\
$\mathrm{C}$ & -2.917630000 & -1.086379000 & -0.271810000 \\
$\mathrm{C}$ & -3.126930000 & -0.555999000 & -1.555030000 \\
$\mathrm{C}$ & -3.753180000 & -0.657269000 & 0.794180000 \\
$\mathrm{C}$ & -4.127500000 & 0.386401000 & -1.737990000 \\
$\mathrm{C}$ & -4.766190000 & 0.286461000 & 0.613350000 \\
$\mathrm{C}$ & -4.937440000 & 0.804911000 & -0.663040000 \\
$\mathrm{H}$ & 0.096390000 & 2.939801000 & 2.444760000 \\
$\mathrm{H}$ & 0.799280000 & -1.718019000 & 0.614460000 \\
$\mathrm{H}$ & 0.860820000 & 4.272391000 & -2.045790000 \\
$\mathrm{H}$ & 1.175670000 & 0.001641000 & -1.062770000 \\
$\mathrm{O}$ & -3.320000000 & -1.303909000 & 1.936930000 \\
$\mathrm{O}$ & 0.008740000 & 2.121051000 & -1.904260000 \\
$\mathrm{O}$ & 0.558090000 & 4.156921000 & -1.131260000 \\
$\mathrm{O}$ & 1.936330000 & -2.145829000 & -2.296850000 \\
$\mathrm{H}$ & 2.000020000 & -3.635499000 & -0.601080000 \\
$\mathrm{H}$ & & &
\end{tabular}


TABLE S44: Geometry for WW-Z1.

\begin{tabular}{lrrr} 
Element & $\mathrm{X}$ & $\mathrm{Y}$ & $\mathrm{Z}$ \\
\hline $\mathrm{C}$ & 0.654048000 & 2.553413000 & 0.460720000 \\
$\mathrm{C}$ & 0.677694000 & 2.353920000 & 1.838013000 \\
$\mathrm{C}$ & 0.771952000 & -1.011866000 & -0.081578000 \\
$\mathrm{C}$ & 1.743557000 & -2.069740000 & -0.639811000 \\
$\mathrm{C}$ & 3.143478000 & -1.789216000 & -0.193751000 \\
$\mathrm{C}$ & 3.670180000 & 0.548582000 & -1.256416000 \\
$\mathrm{C}$ & 3.906174000 & -0.597150000 & -0.478573000 \\
$\mathrm{C}$ & 3.920689000 & -2.566317000 & 0.635442000 \\
$\mathrm{C}$ & 4.647104000 & 1.529861000 & -1.316599000 \\
$\mathrm{C}$ & 5.141859000 & -0.720255000 & 0.209373000 \\
$\mathrm{C}$ & 5.861182000 & 1.391120000 & -0.615952000 \\
$\mathrm{C}$ & 6.127516000 & 0.266856000 & 0.151263000 \\
$\mathrm{C}$ & -0.451050000 & 1.854985000 & 2.524249000 \\
$\mathrm{C}$ & -0.530783000 & 2.251027000 & -0.213059000 \\
$\mathrm{C}$ & -0.705828000 & -1.366794000 & -0.303824000 \\
$\mathrm{C}$ & -1.619413000 & 1.534174000 & 1.844209000 \\
$\mathrm{C}$ & -1.675444000 & 1.732760000 & 0.457029000 \\
$\mathrm{C}$ & -2.165046000 & 1.962339000 & -1.732226000 \\
$\mathrm{C}$ & -2.703111000 & 1.554022000 & -0.538553000 \\
$\mathrm{C}$ & -4.077939000 & 1.008308000 & -0.310467000 \\
$\mathrm{C}$ & -4.109719000 & -0.526814000 & -0.161391000 \\
$\mathrm{C}$ & -5.482373000 & -1.070339000 & 0.204152000 \\
$\mathrm{H}$ & 0.148371000 & -1.432663000 & 1.800366000 \\
$\mathrm{H}$ & 0.850157000 & 0.082591000 & 1.718209000 \\
$\mathrm{H}$ & 0.983342000 & -0.037952000 & -0.512126000 \\
$\mathrm{H}$ & 1.428934000 & -3.065942000 & -0.314574000 \\
$\mathrm{~N}$ & -1.121587000 & -1.223541000 & -1.486897000 \\
$\mathrm{H}$ & -1.351042000 & -1.752551000 & 0.690219000 \\
$\mathrm{O}$ & -5.969582000 & -0.482304000 & 1.302213000 \\
$\mathrm{O}$ & -6.040917000 & -1.940655000 & -0.415563000 \\
$\mathrm{H}$ & & &
\end{tabular}

